\title{
LA DÉTERMINATION DE L'AGE PAR SCALIMÉTRIE CHEZ LE SAUMON ATLANTIQUE (SALMO SALAR) DANS SON AIRE DE RÉPARTITION MÉRIDIONALE : UTILISATION PRATIQUE ET DIFFICULTÉS DE LA MÉTHODE
}

\author{
Compte rendu des synthèses et discussions de l'atelier de lecture d'écailles \\ (Rennes, 1 er au 4 avril 1985).
}

\section{J.L. BAGLINIERE (1)}

Avec la participation de : BOMASSI P. (2), BOUSOUET B. (3), CHANCEREL F. (4), DE PONTUAL H. (12), DUMAS J. (5), EUZENAT G. (6), FONTENELLE G. (7), FOURNEL F. (6), GAYOU F. (8), LUQUET J.F. (4), MAISSE G. (9), MARTIN VENTURA J.A. (10), MARTY A. (3), NIHOUARN A. (11), PORCHER J.P. (11), PREVOST E. (1), PROUZET P. (12), PUSTELNIK G. (8). RICHARD A. (11) et TROADEC H. (12).

(1) Laboratoire d'Ecologie Hydrobiologique - INRA - 65, route de St-Brieuc - 35042 RENNES CEDEX.

(2) Délégation Régionale du Conseil Supérieur de la Pêche Auvergne-Limousin - 84, avenue du Puy de Dôme - 63000 CLERMONT-FERRAND.

(3) Délégation Régionale du Conseil Supérieur de la Pêche Midi-Pyrénées - Building des Pyrénées 64000 PAU

(4) Délégation Régionale du Conseil Supérieur de la Pêche Région de Poitiers - 112, faubourg de la Cueille - 86000 POITIERS.

(5) Laboratoire d'Ecologie des Poissons - INRA - St-Pée-sur-Nivelle - 64310 ASCAIN.

(6) Délégation Régionale du Conseil Supérieur de la Pêche Picardie-Haute Normandie - 3, rue Sainte Marie - 60200 COMPIËGNE.

(7) Laboratoire de Biologie et Ecologie marine - ENSA - 65, route de St-Brieuc - 35042 RENNES CEDEX.

(8) Délégation Régionale du Conseil Supérieur de la Pêche Région Toulouse - Chemin des Maraîchers - 31400 TOULOUSE.

(9) Laboratoire de Physiologie des Poissons - INRA - Faculté des Sciences de Beaulieu - $\mathbf{3 5 0 4 2}$ RENNES CEDEX.

(10) Consejeria de Agricultura y Pesca del Principado de Asturias, Seccion de Conservacion de la Naturaleza - Uria 10 - 33071 OVIEDO - ESPANA.

(11) Délégation Régionale du Conseil Supérieur de la Pêche Bretagne-Basse Normandie - 84, rue de Rennes - 35510 CESSON SEVIGNE.

(12) IFREMER, BP 337 - 29273 BREST CEDEX.

\section{RESUME}

Ce rapport confirme les critères d'interprétation scalimétrique utilisés pour âger le saumon atlantique en eau douce et en mer à l'aide d'exemples typiques. Il synthétise également les difficultés rencontrées pour la détermination d'âge des populations naturelles et d'élevage en les illustrant à l'aide d'exemples atypiques. Enfin, il propose un certain nombre de recommandations pour permettre une meilleure connaissance des populations de saumon atlantique dans son aire de répartition méridionale.

\section{AGE DETERMINATION OF ATLANTIC SALMON (SALMO SALAR) BY SCALE READING IN SOUTH . DISTRIBUTION AREA : USE AND DIFFICULTIES OF THIS METHOD.}

\section{SUMMARY}

This report confirms interpretation criteria used in scale reading for age determination of Atlantic Salmon in freshwater and in sea water with typical examples. The difficulties for age determination of wild and reared populations by showing atypical examples are presented. Finally some recommandations to a better knowledge of Atlantic salmon population in south distribution area are proposed. 


\section{INTRODUCTION}

Un premier atelier de lecture d'écailles du saumon atlantique a été organisé à Aberdeen en avril 1984 par le laboratoire de Montrose du DAFS (Departement de l'Agriculture et des Pêcheries d'Ecosse), suite à une initiative du Conseil International pour l'Exploration de la Mer. Huit pays étaient représentés dont la France par J.L. BAGLINIERE. Cet atelier avait pour but d'homogénéiser les critères de détermination de l'âge par scalimétrie chez le saumon atlantique. Il a donné lieu à un rapport précisant un certain nombre de règles pour l'interprétation scalimétrique et donnant des recommandations afin de poursuivre les recherches dans ce domaine (BAGLINIËRE, com. pers.).

Ce même besoin d'homogénéiser l'interprétation des écailles de saumon atlantique se faisant sentir également en France, un atelier de lectures d'écailles de cette espèce s'est réuni à Rennes au mois d'avril 1985. Il regroupait toutes les personnes des différents organismes scientifiques et délégations du Conseil Supérieur de la Pêche directement concernés par ce problème. De fait, toutes les régions encore productrices de saumon en France (Loire-Allier, Adour-Gaves, Bretagne et Basse-Normandie) étaient représentées. L'Espagne a également participé à cet atelier par l'envoi d'échantillons d'écailles. Ainsi, les critères scalimétriques de détermination d'âge chez le saumon atlantique ont pu être comparés dans son aire de répartition méridionale.

Cet article fait la synthèse de l'ensemble des problèmes et difficultés soulevés pour la détermination de l'âge du saumon atlantique par scalimétrie.

\section{1 - PResentation des Regions productrices en SAUMON (Fig. 1).}

En France, trois régions principales ont des rivières fréquentées par le saumon atlantique :

- le Massif Armoricain avec vingt-cinq rivières,

- la région Centre avec l'axe Loire-Allier,

- la région du Sud-Ouest avec l'axe Adour-Gaves-Nive, la Nivelle et la Dordogne. A l'ensemble de ces rivières, il faut en ajouter quelques autres telles l'Orne (Basse-Normandie : RICHARD, com. pers.) et la Bresle (Haute-Normandie : FOURNEL, EUZENAT et FAGARD, 1985) où l'on observe des remontées régulières.

En Espagne, il existe vingt-cinq rivières réparties dans quatre provinces de la façade atlantique (MARTIN VENTURA, 1985). Les deux rivières étudiées, Esva et Narcea, appartiennent à la province des Asturies. Sur la rivière Narcea, seules quelques écailles typiques représentant les divers âges d'eau douce et de mer ont été analysées.

Les caractéristiques biologiques générales des populations de saumon atlantique des différentes régions sont présentées : spectre de classe d'âge d'eau douce et de mer, taille aux stades smolt et adulte.

\section{2 - COMPTE-RENDU DE L'ATELIER INTERNATIONAL DE LECTURE D'ÉCAILlES.}

Lors de cet atelier, il a été décidé (BAGLINIËRE, com. pers.) :

-_ une localisation standard pour le prélèvement d'écailies : 1 à 6 rangs au-dessus de la ligne latérale et sur une ligne joignant l'attache postérieure de la nageoire dorsale à l'attache antérieure de la nageoire anale.

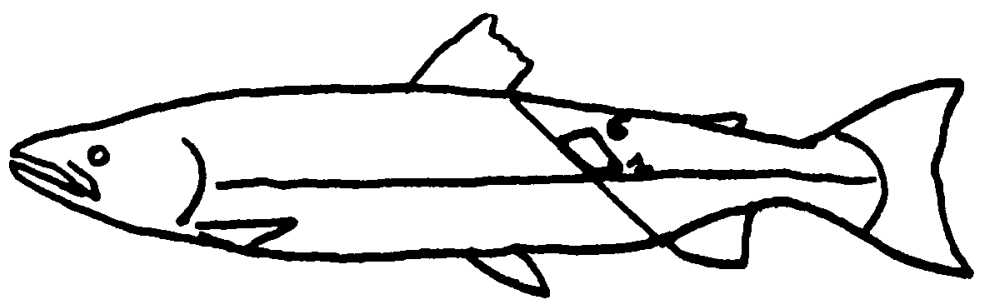

- si possible d'imprimer les écailles sur des films plastiques (PVC). Les cinq écailles choisies doivent présenter un nucleus de diamètre $<0,5 \mathrm{~mm}$. Une bonne impression permet une meilleure lecture et un examen plus fin des structures internes de l'écaille (comptage des circuli).

Par ailleurs, un certain nombre de critères et de définitions ont été précisées dont les plus importantes sont :

- l'existence de trois zones sur une écaille de saumon adulte : rivière, transition et mer,

- la zone annuelle est définie comme une région concentrique de l'écaille se référant à une année de vie.

- une bande est définie comme une région concentrique de l'écaille formée pendant une certaine période de l'année. Elle est claire si les circuli sont espacés, sombre s'ils sont resserrés,

- l'annulus est défini comme la limite théorique entre deux zones successives annuelles correspondant au premier circulus discontinu suivant une bande sombre,

- la reprise de croissance est caractérisée comme une région de circuli espacés suivant un annulus,

- yn "check" correspond à un changement de mode de croissance en relation avec les conditions 


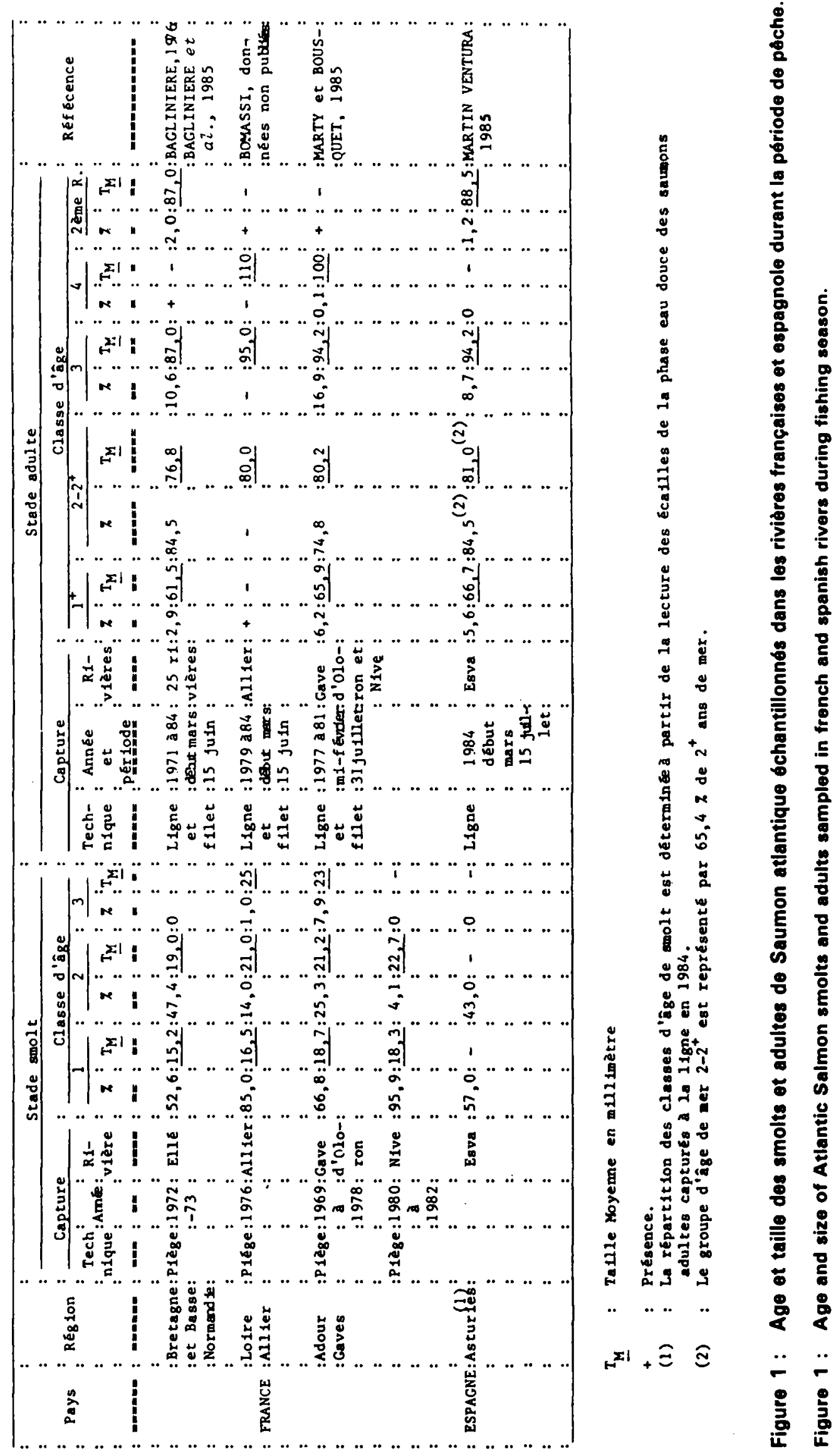




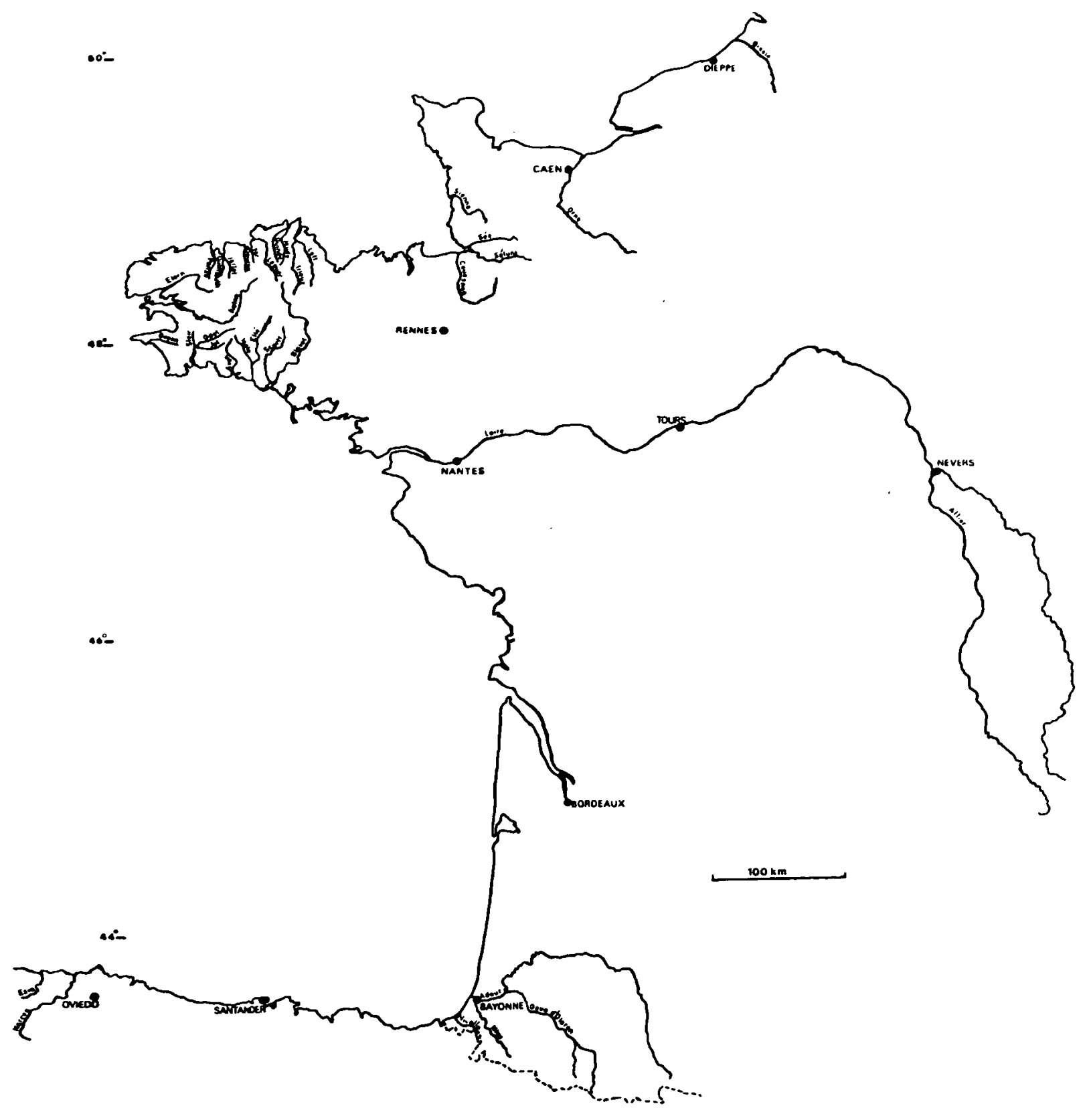


environnementales en hiver comme en été et se présentant sous forme de bande sombre. II semble apparaitre chez des individus en phase ascendante de forte croissance en relation avec les conditions trophiques (BILTON \& ROBINS, $1971 a, b, c)$.

En ce qui concerne la détermination de l'âge d'eau douce, aucune règle n'a été avancée. Par contre, en phase marine des normes ont été établies pour différencier les hivers des checks. Ce sont les suivantes:

- est considérée comme premier hiver toute bande sombre donnant une longueur rétromesurée (rétromesure linéaire) du poisson comprise entre 40 et 60 centimètres.

- est considérée comme second hiver toute bande sombre apparaissant à une longueur rétromesurée ou égale à 1.55 fois celle du premier hiver,

- est considérée comme troisième hiver toute bande sombre apparaissant à une longueur rétromesurée supérieure ou égale à 1.2 fois celle du second hiver.

Pour les poissons ayant déjà frayé une fois, il est considéré que la période de remontée et la structure du bord de l'écaille restent les mêmes que celles observées lors du premier retour. Toutefois. cette règle reste moins impérative que les précédentes.

\section{3 - EXAMEN SCALIMETTRIQUE}

\subsection{Age d'eau douce}

La détermination de l'âge s'est faite à partir d'écailles de juvéniles (parr et smolt) et d'adultes. Des exemples types des différents âges d'eau douce ( 1 à 3 ans) sont présentés. On constate (Fig. 2 à 31 ) :

- une croissance semblable pour les rivières Esva, Narcea, Allier et celles de Bretagne Basse-Normandie,

- une croissance en moyenne nettement plus élevée dans les rivières du Sud-Ouest de la France (effet de la latitude tamponné par celui de l'altitude et/ou relation densité - croissance).

- la présence de marques de fraie au stade parr (ligne continue autour de l'écaille, érosion et/ou recoupement de circuli). Initialement elles ont été mises en évidence sur des écailles de parrs, de smolts et d'adultes du saumon atlantique des rivières du Massif Armoricain (MAISSE \& BAGLINIERE, 1983 ; BAGLINIËE \& MAISSE, 1985).

A titre de comparaison les phases eau douce d'écailles de saumon adultes capturés sur des rivières plus nordiques sont montrées. Leur examen fait apparaître une croissance d'autant plus faible que l'on monte en latitude et souvent une absence de zone de transition (Fig. 32 à 35).

Quelques difficultés pour déterminer l'âge d'eau douce sont apparues essentiellement à partir des écailles d'adultes. Elles concernent :

- des individus à croissance continue (hiver absent ou difficilement discernable) ou présentant plusieurs rythmes de croissance sans différence notable d'espacement de circuli (apparition de checks, faux anneaux, etc.). Dans les deux cas la ou les bandes hivernales ne sont pas facilement repérables (Fig. 36 à 47).

- des individus âgés de 1 ou 2 ans présentant dans leur phase smolt un check net pouvant se confondre avec une bande hivernale (Fig. 48 à 55). La présence d'un tel phénomène paraît d'autant plus probable et marquée que la distance à parcourir par le smolt est plus grande (cas de l'Allier 600 à $850 \mathrm{~km}$ ).

Plusieurs critères peuvent être utilisés pour résoudre les problèmes posés par de tels cas :

- la comparaison avec des écailles de parrs et de smolts de la cohorte dont l'adulte est issu,

- une croissance de l'écaille en moyenne plus importante lors de la deuxième année chez les individus âgés de plus d'un an,

- la taille ou le nombre de circuli de la partie eau douce de l'écaille. Ces deux paramètres augmentent avec l'âge surtout entre un et deux ans,

- la présence de marque de fraie. Elle est trouvée essentiellement sur les individus de 2 ou 3 ans,

- l'épaisseur du check (généralement faible) en comparant celle des circuli et leur espacement avant et après le "faux anneau".

Chez les poissons d'élevage (intensif : pisciculture ou extensif : ruisseau pépinière), on observe d'une part un grand nombre d'écailles régénérées au centre et, d'autre part, des structures identiques mais souvent plus anarchiques que celles des cas particuliers constatés chez les poissons sauvages (différents rythmes de croissance, présence de un ou plusieurs faux anneaux, absence de bande hivernale) (Fig. 56 a 61). De telles structures ne permettent pas d'åger le saumon d'élevage dans sa phase en eau douce en absence de marquage au stade smolt.

\subsection{Age de mer}

Des exemples typiques des différents âges de mer sont présentés depuis le castillon précoce $\left(1^{+}\right.$ de mer) jusqu'au poisson de troisième remontée (Fig. 62 a 76). En général, pour âger les poissons en 
mer, moins de difficultés sont apparues que pour la phase eau douce ; elles concernent :

- la présence de checks principalement situés dans la deuxième année de vie marine. Ces checks peuvent être soit des dédoublements du premier hiver, soit des bandes sombres concentriques indépendantes. lls peuvent également se former en bordure d'écailles en relation avec la maturation sexuelle (Fig. 77 a 86 ),

- I'absence soit du premier (croissance continue), soit du second ou du troisième hiver (bordure de l'écaille). Ce dernier cas concerne les poissons remontant en début d'année (date anniversaire $1^{\text {er }}$ avril) (Fig. 87 à 92). A ces exemples peut s'ajouter le cas de castillon présentant peu ou pas de reprise de croissance après le premier hiver en mer (Fig. 93),

- un phénomène d'érosion partielle ayant eu lieu lors d'un hiver et visible essentiellement sur les champs latéraux de l'écaille. Il constitue une fausse marque de fraie (Fig. 94 et 95),

- un phénomène d'érosion périphérique en liaison avec la maturation sexuelle. Il est fréquemment observé chez les poissons échantillonnés en fin d'année (la fraie se situe généralement en décembre) et chez les bécards ayant survécu à leur fraie et redescendant vers la mer. II est nettement plus accentué chez les mâles que chez les femelles. Généralement, le seul examen de l'écaille ne permet pas de connaître l'âge de mer correct du poisson (Fig. 96 à 99). Le même problème se pose pour déterminer l’åge de première remontée des individus ayant fait un deuxième séjour en mer (Fig. 100).

\section{intervenir :}

Pour résoudre ces cas particuliers et âger correctement le poisson, différents éléments peuvent

- I'utilisation des normes proposées à l'atelier international d'Aberdeen pour identifier et positionner les bandes hivernales. De telles normes doivent être utilisées principalement dans les cas difficiles (confusion d'un check et d'un hiver). Cependant, elles ont été vérifiées en règle générale sur des échantillons d'écailles de saumons de toutes les régions présentées ici,

- la connaissance de nombreux parametres intéressant la capture : la date, la technique (ligne. poisson mort, piégeage montée ou descente), la taille, le poids, la couleur de la robe (blanche. mordorée ou rose, rouge foncé ou orange). la présence de copépodes parasites. Quelquefois ces données restent insuffisantes et la scalimétrie montre ses limites d'utilisation. Dans le cas de poissons morts, la détermination de l'âge pourrait se faire à partir des otolithes,

- la définition d'une marque de fraie chez un saumon adulte : présence d'une ligne continue concentrique (elle doit être visible sur la partie extérieure de l'écaille), discontinuité dans le positionnement des circuli situés avant et après la ligne continue, une très forte reprise de croissance après cette marque (Fig. 100). Pour les poissons de deuxième remontée, dans un premier temps, il est admis que la période et la structure de l'écaille sont les mêmes lors des première et seconde remontées (Fig. 101 à 103). Dans un deuxième temps, il a été décidé de vérifier cette hypothèse pour les poissons ayant survécu à leur fraie à nos latitudes.

Enfin, il n'apparaît aucune difficulté particulière pour åger en mer les poissons d'élevage. 


\section{LEGENDE DES FIGURES POUR LA PHASE EN EAU DOUCE}

Stade - Rivière (affluent) - Région - Pays - Date et Technique de capture - Longueur totale (mm) Sexe (supposé) - Age - Interprétations - Observations

\section{LEGEND OF PLATES FOR FRESHWATER STAGE}

Stage - River (Tributary) - Area - Country - Date and Technique of catch - Total length (mm) - Sex (supposed) - Age - Interpretation - Observations. 


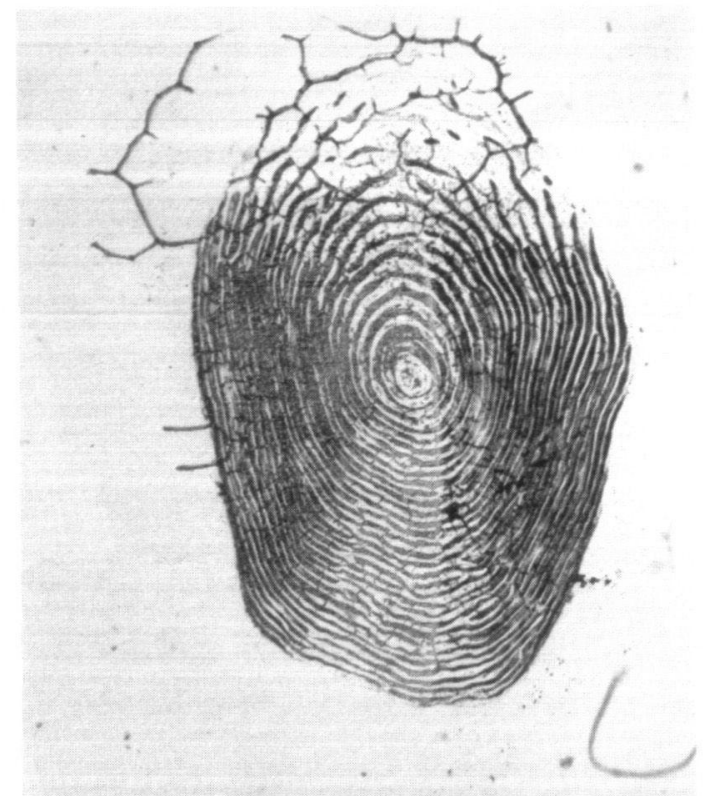

Figure 2 : Parr, Scorff, Bretagne, France 25.09.78, pêche électrique. $125,0^{+}$.

Figure 2 : Parr, Scorff, Brittany, France. 25.09.78, electrofishing, 125 , $0^{+}$.

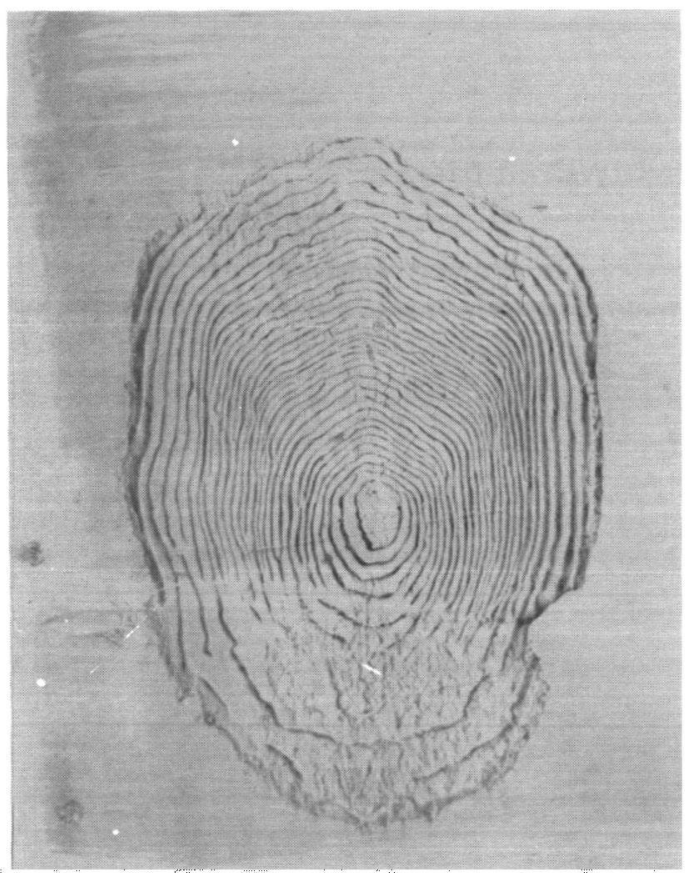

Figure 4: Smolt, Sélune. (L'Oir), BasseNormandie, France, 2.05.85, piège de descente, 140, $1^{+}$.

Figure 4: Smolt. Sélune (L'Oir), Low Normandy, France, 2.05.85. downstream trap, $140,1^{+}$.

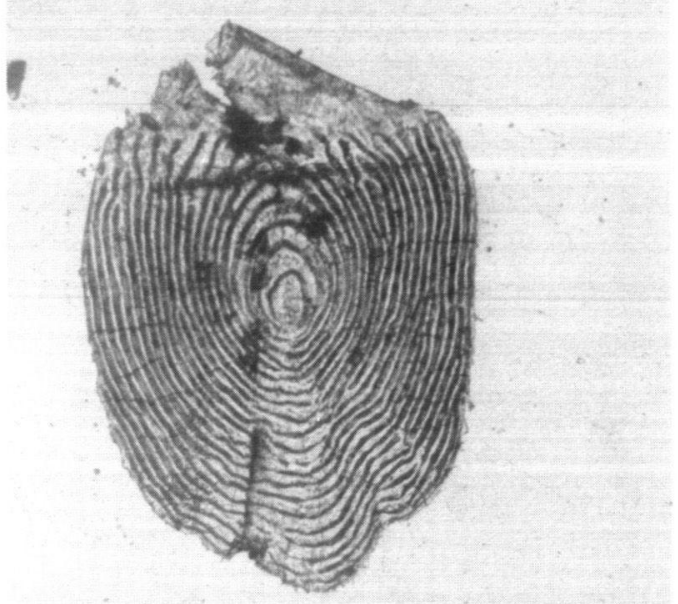

Figure 3 : Parr, Gave d'Oloron. SudOuest, France, 13.10.78, pêche électrique, $115,0^{+}$.

Figure 3 : Parr, Gave d'Oloron, South West, France, 13.10.78, electrofishing, $115,0^{+}$.

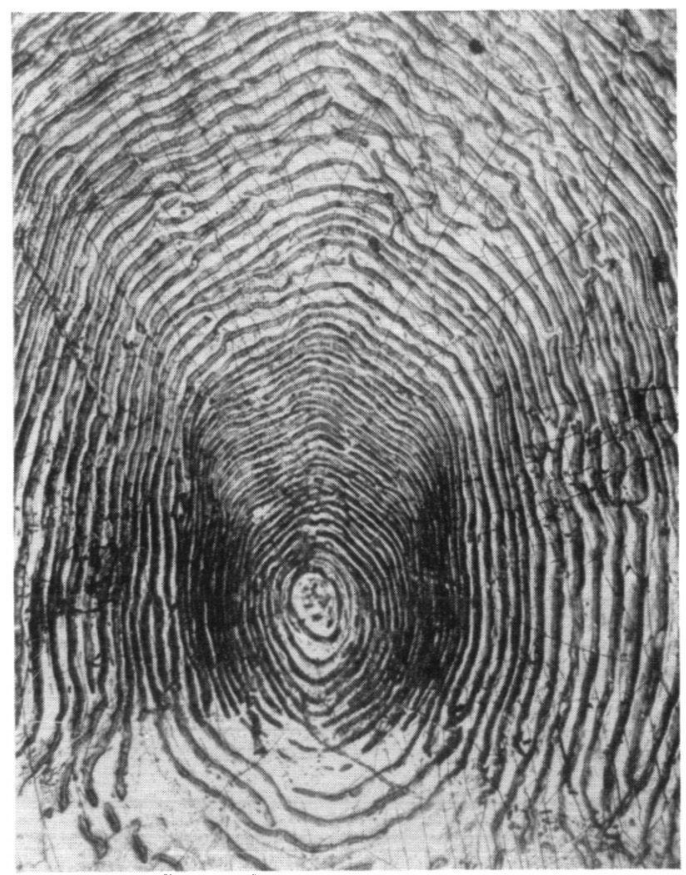

Figure 5: Adulte, Blavet (Tymat), Bretagne. France, 1.12.73, piège de montée, $631,1^{+}$.

Figure 5 : Adult, Blavet (Tymat) Brittany, France, 1.12.73, upstream trap. $631,1^{+}$. 


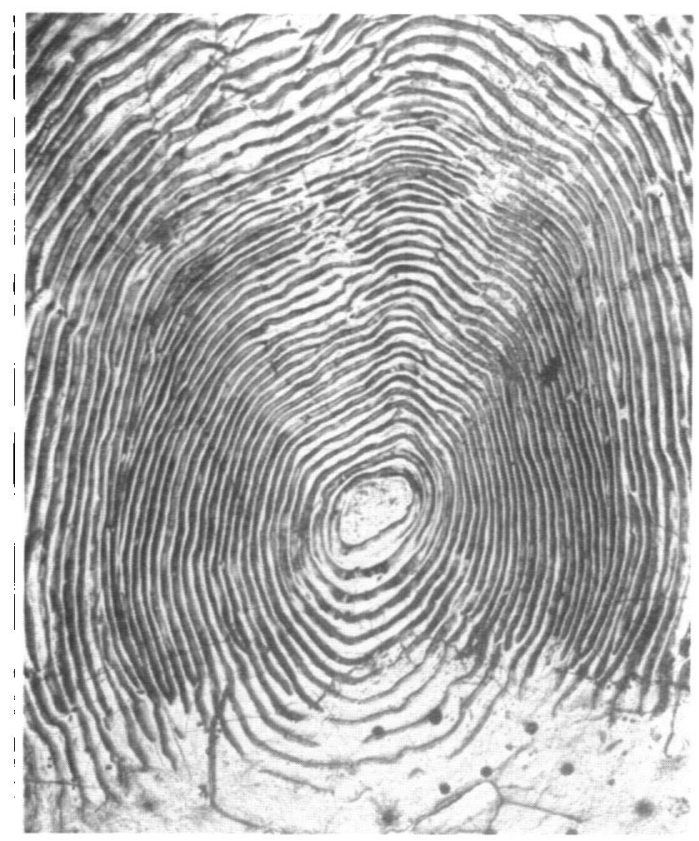

Figure 6 : Adulte, Odet, Bretagne, France, 7.04.75, ligne, 830, $1^{+}$, forte croissance en eau douce et faible reprise de croissance.

Figure 6 : Adult, Odet, Brittany, France, 7.04.75, rod, 830, $1^{+}$, high growth in freshwater and small plus growth.

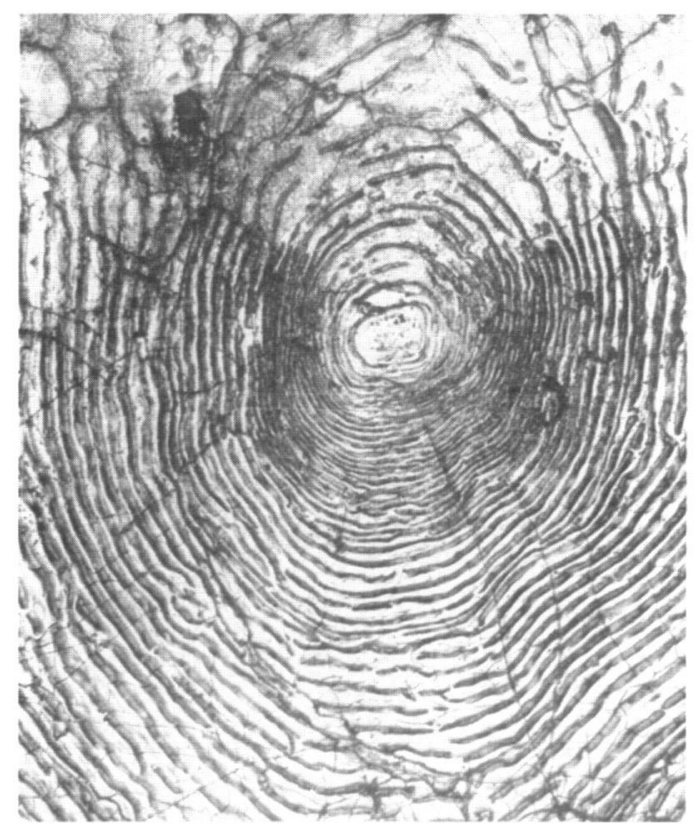

Figure 8: Adulte, Allier, Centre, France, 3.05.80, ligne, 900, $1^{+}$, phase smolt bien individualisée.

Figure 8: Adult, Allier, Centre, France, 3.05.80, rod, 900, $1^{\text {}}$, smolt stage well visible.

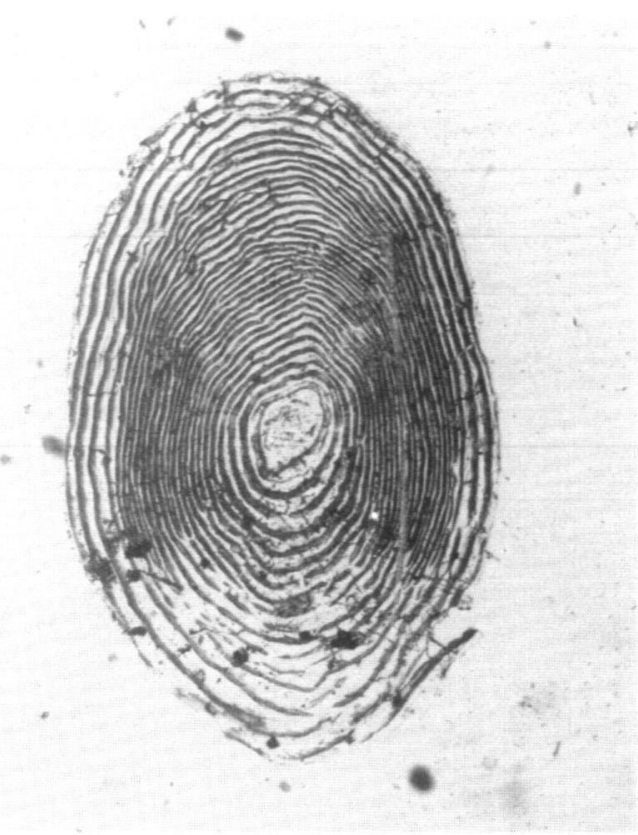

Figure 7: Smolt, Allier, Centre, France, 6.04.76, piège de descente, 177. $1^{+}$.

Figure 7 : Smolt, Allier, Centre, France, 6.04.76, downstream trap. 177, $1^{+}$.

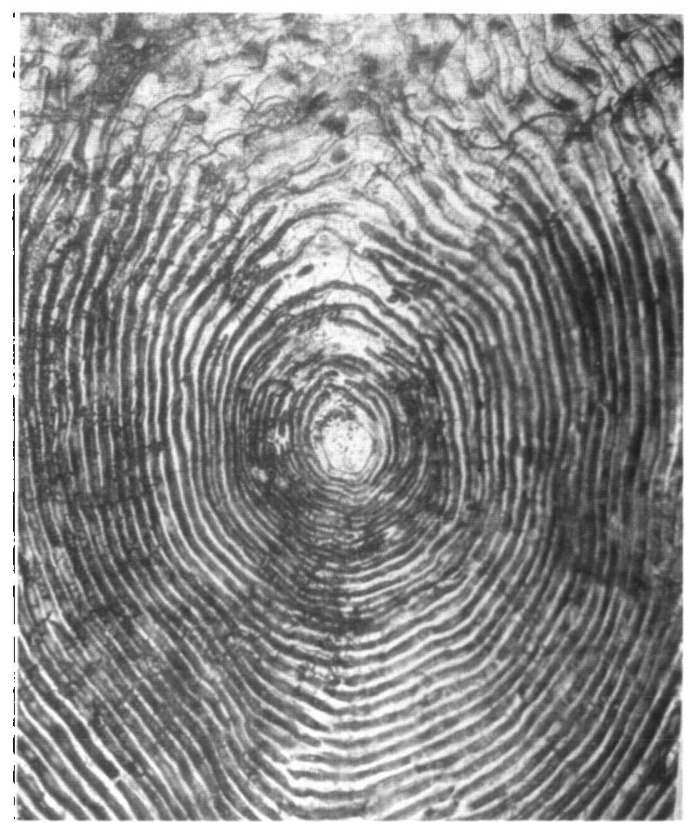

Figure 9: Adulte, Allier, Centre, France, 31.03.80, ligne, 970, $1^{+}$, petit smolt.

Figure 9 : Adult, Allier, Centre, France, 31.03.80, rod, $970,1^{+}$, small smolt. 


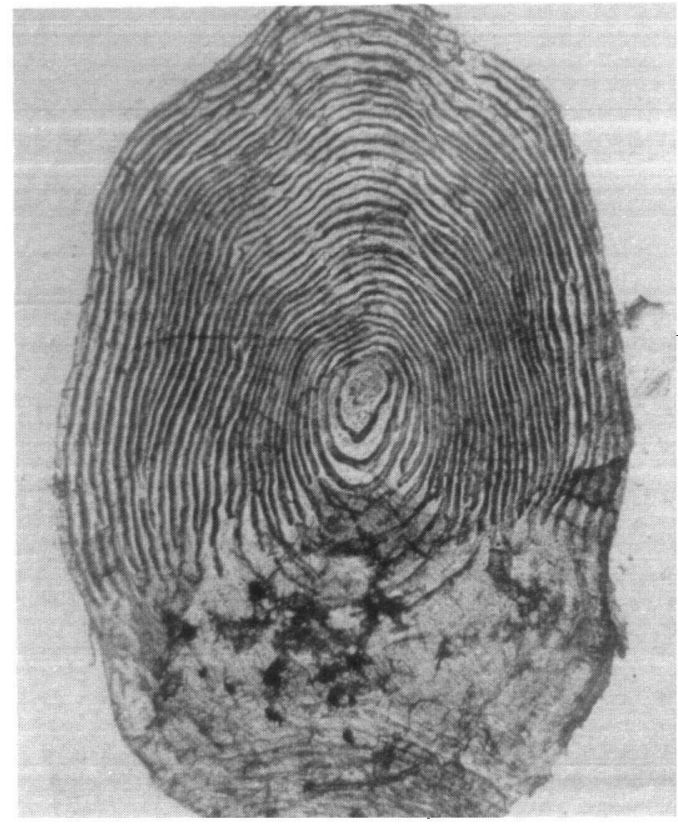

Figure 10 : Smolt, Gave d'Oloron, SudOuest, France, 11.04.78, piège de descente, $177,1^{+}$.

Figure 10 : Smolt, Gave d'Oloron, South West, France, 11.04.78. downstream trap, 177, $1^{+}$.

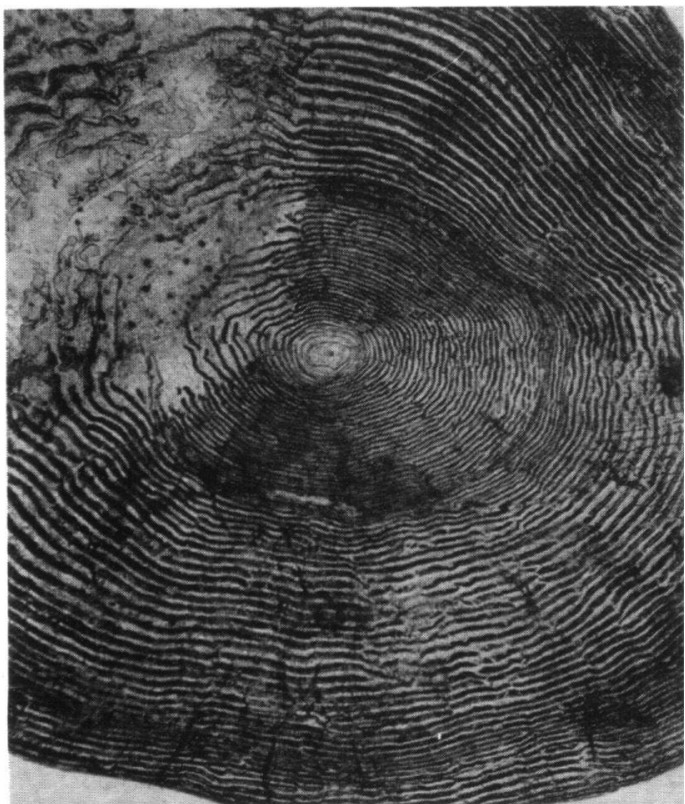

Figure 12 : Adulte, Adour, Sud-Ouest, France, 1.06.77, ligne, 680, 1 très gros smolt, possible marque de fraie lors du premier hiver en eau douce.

Figure 12 : Adult, Adour, South West, France, 1.06.77, $1^{+}$, very big smolt, possible spawning mark at first freshwater winter.

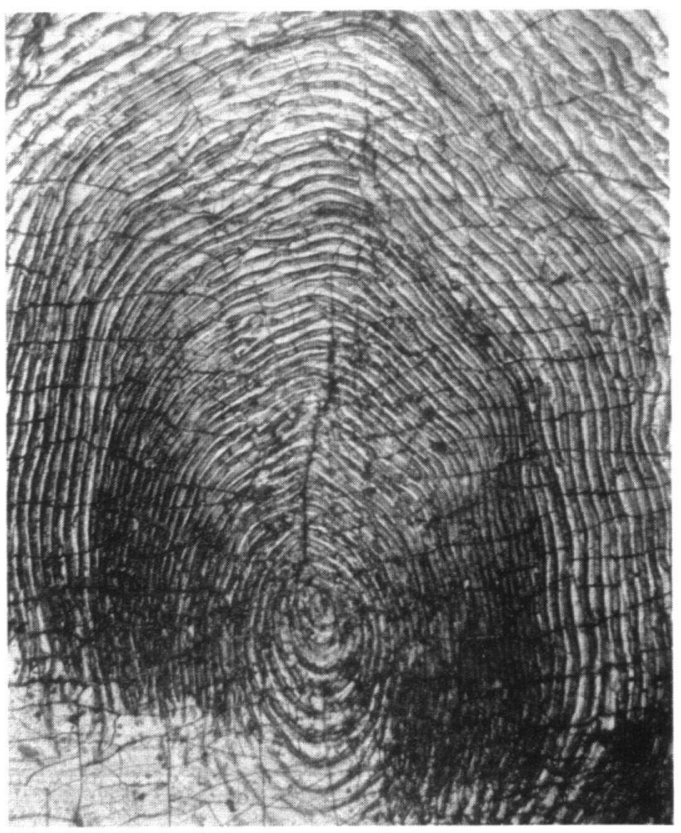

Figure 11 : Adulte, Gave d'Oloron, SudOuest, France, 24.05.77, ligne, $920,1^{+}$, phase smolt bien individualisée.

Figure 11 : Adult, Gave d'Oloron, South West, France, 24.05.77, rod, $920,1^{+}$, smolt stage well visible.

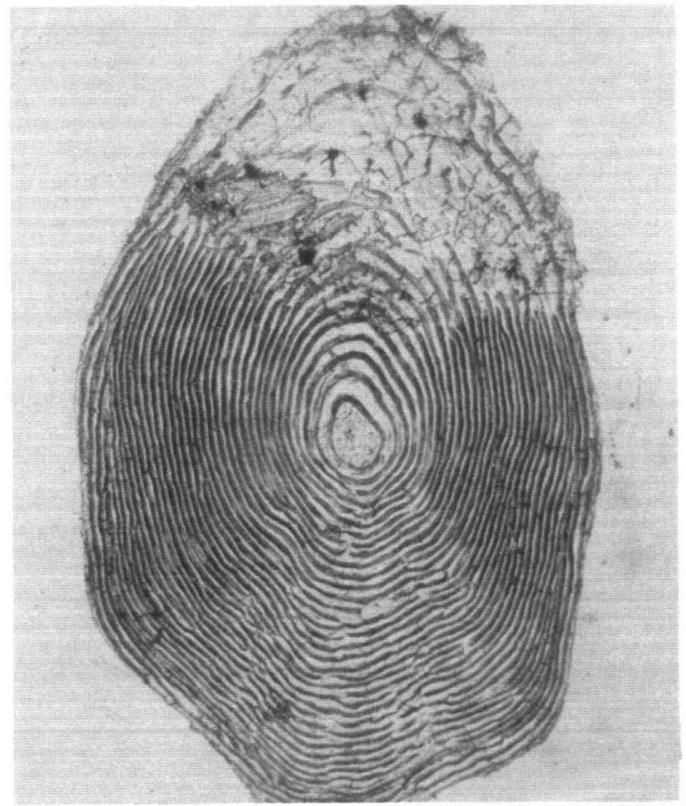

Figure 13 : Smolt, Nive, Sud-Ouest, France, 6.04.81, piege de descente, $189.1^{+}$.

Figure 13 : Smolt, Nive, South West, France, 6.04.81, downstream trap, 189, $1^{+}$. 


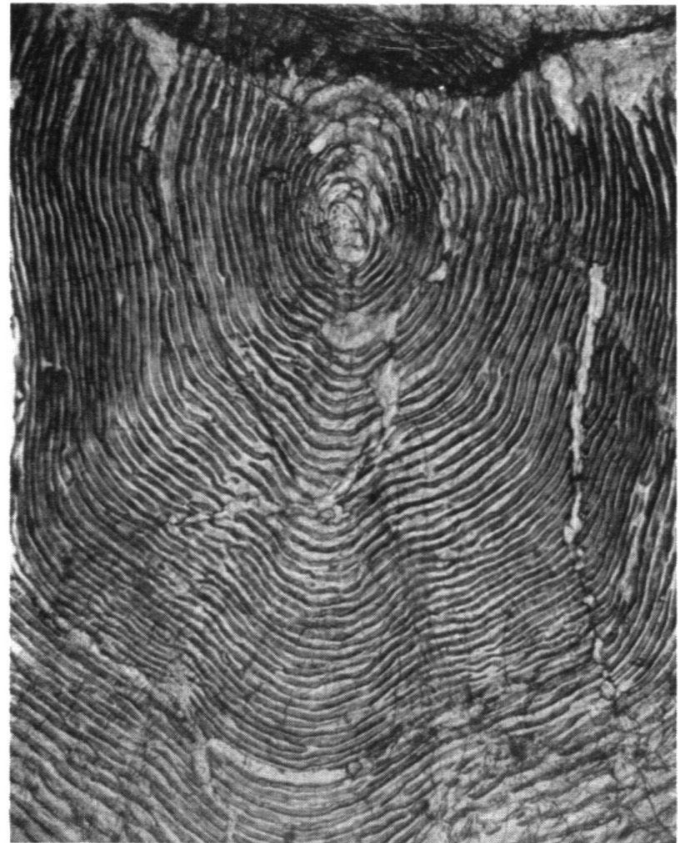

Figure 14 : Adulte. Nivelle, Sud-Ouest, France, 21.08.81, pêche électrique, $670,1^{+}$.

Figure 14 : Adult, Nivelle, South West, France, 21.08.81, electrofishing, 670, 1 .

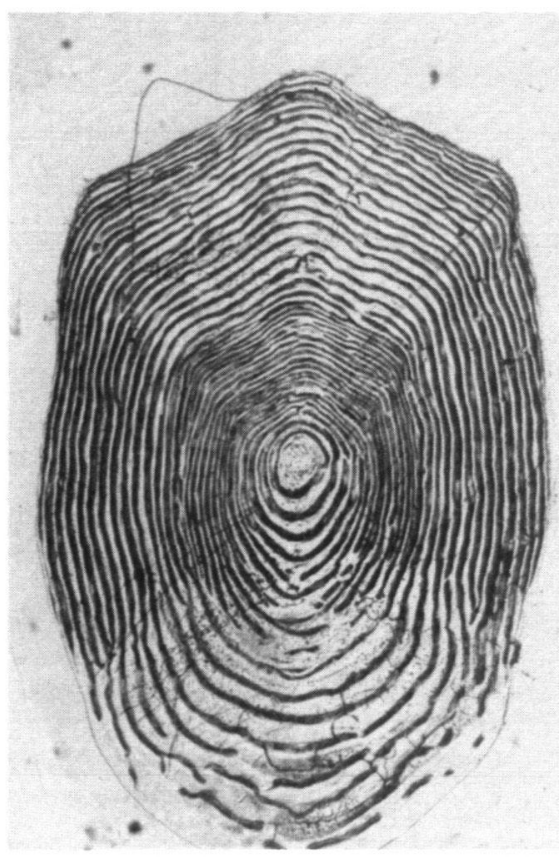

Figure 16 : Parr, Scorf, Bretagne, France, 26.09.78, pêche électrique. $151,1^{+}$.

Figure 16 : Parr, Scorff, Brittany, France. 26.09.78, electrofishing. 151 1 .

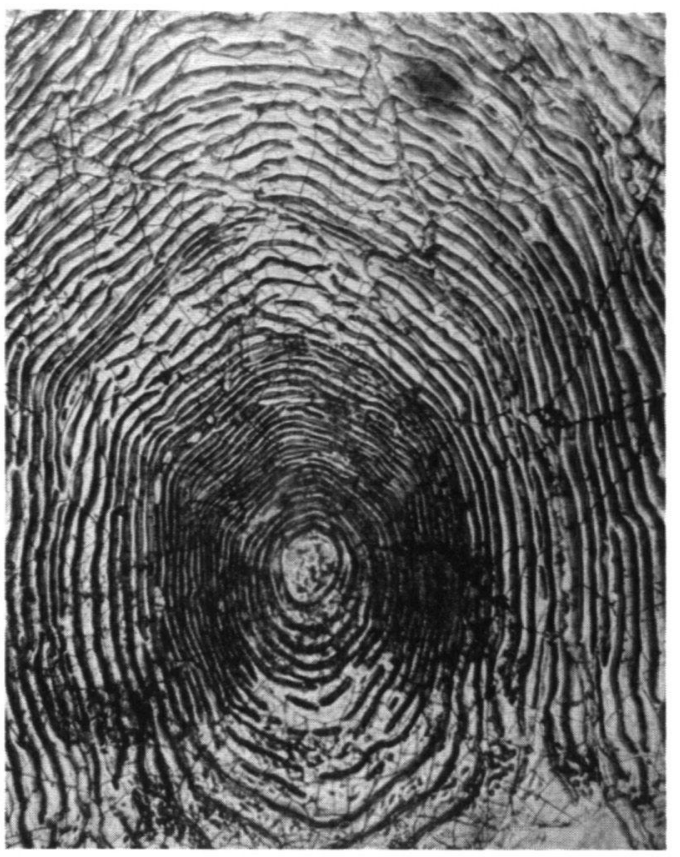

Figure 15 : Adulte, Nivelle, Sud-Ouest, France, 19.11.81, pêche électrique, 676, $\sigma^{\star}, 1^{+}$, cas peu typique de la Nivelle.

Figure 15 : Adult, Nivelle, South West, France, 19.11.81, electrofishing, $676, \sigma^{\circ}, 1^{+}$, no typical example on the Nivelle River.

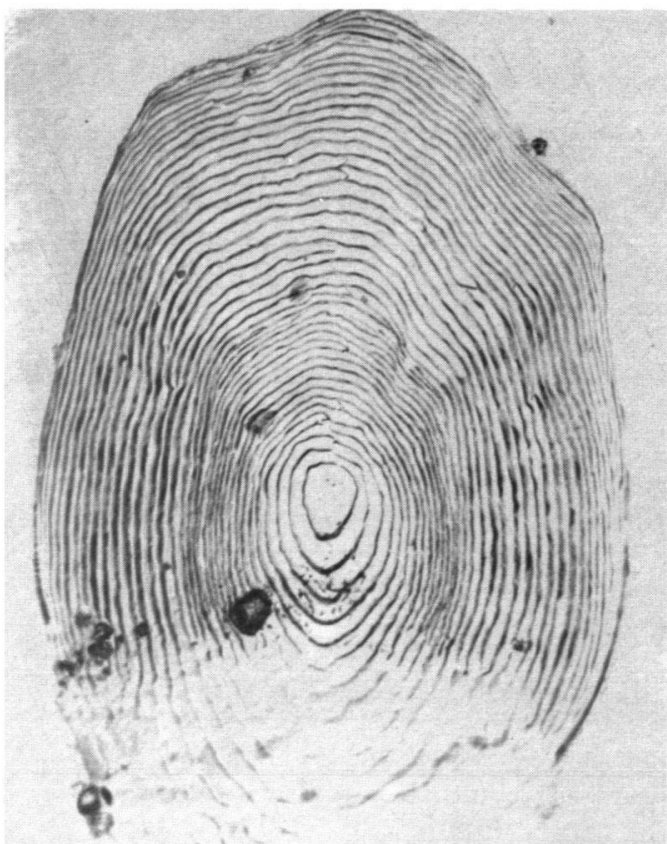

Figure 17 : Smolt, Sélune (L'Oir), BasseNormandie, France, 7.04.85, piège de descente, 190, 2.

Figure 17 : Smolt, Sélune (I'Oir), Low Normandy, France, 7.04.85. downstream trap, 190, 2. 


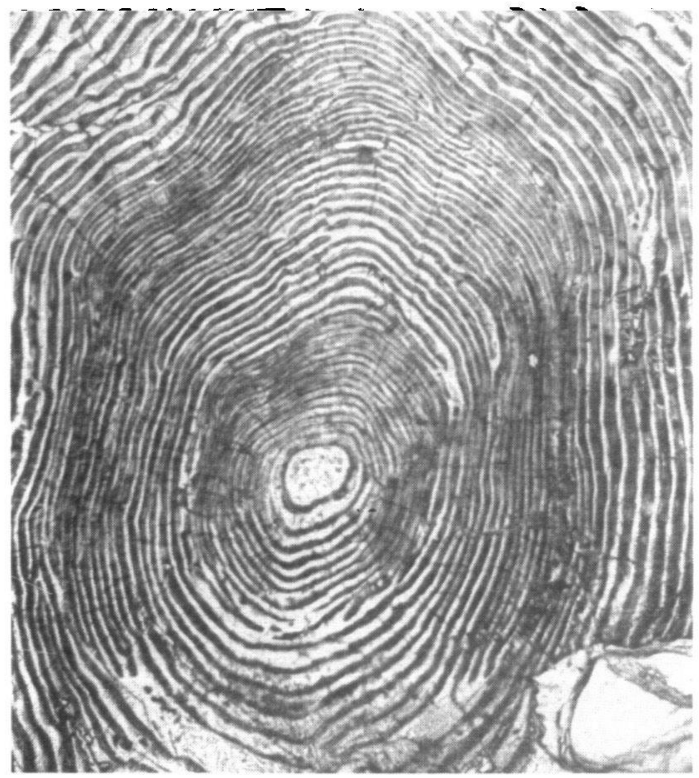

Figure 18: Adulte, Blavet (Tymat). Bretagne, France, 9.12.73, piège de montée, 585, 2.

Figure 18 : Adult, Blavet (Tymat), Brittany, France. 9.12.73, upstream trap, 585, 2.

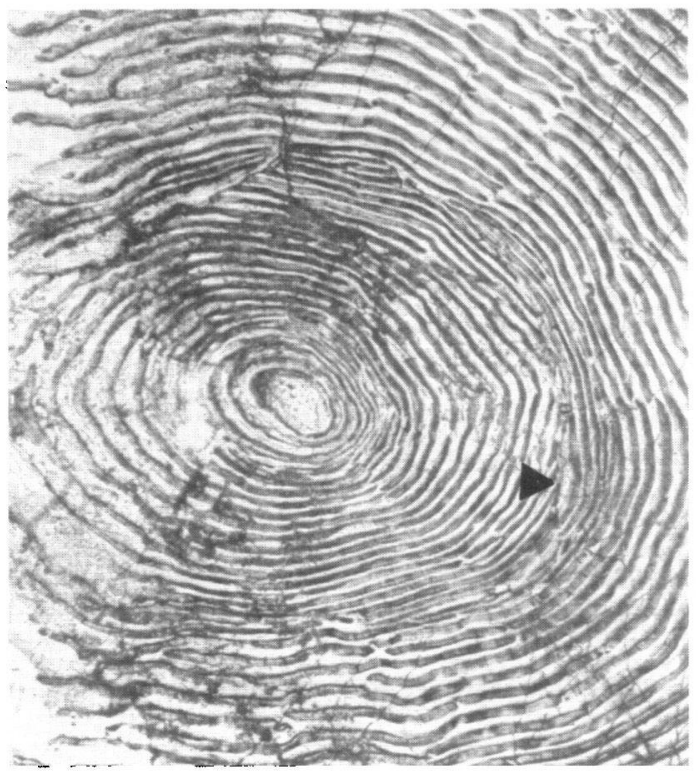

Figure 20 : Adulte, Scorff, Bretagne, France, 29.04.81, 740, (c), 2. marque de fraie lors du deuxième hiver en eau douce (D).

Figure 20 : Adult, Scorff, Brittany. France, 29.04.81, 740 ( ). 2, spawning mark at the second freshwater winter ( $\downarrow$ ).

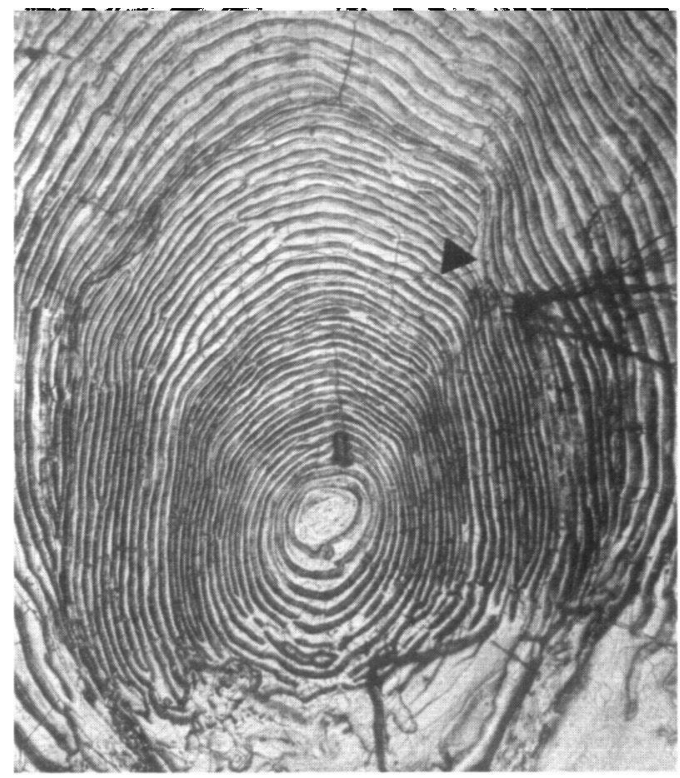

Figure 19 : Adulte, Ellé, Bretagne, France, 18.03.81, ligne 680, $d, 2$, marque de fraie lors du deuxième hiver en eau douce $(\triangleright)$.

Figure 19 : Adult, Ellé, Brittany, France, 18.03.81, rod, 680 , 3 . spawning mark at the second freshwater winter ( $)$.

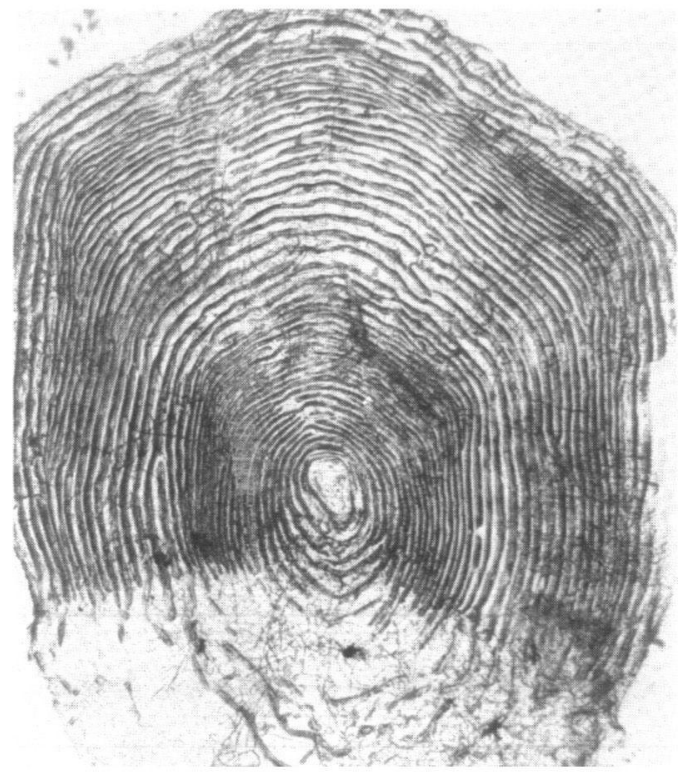

Figure 21 : Smolt, Allier, Centre, France, 6.04.76, piège de descente. $207,2^{+}$, possible marque de fraie lors du deuxième hiver en eau douce.

Figure 21 : Smolt, Allier, Centre, France, 6.04.76, downstream trap, 207, $2^{-}$possible spawning mark at the second freshwater winter. 


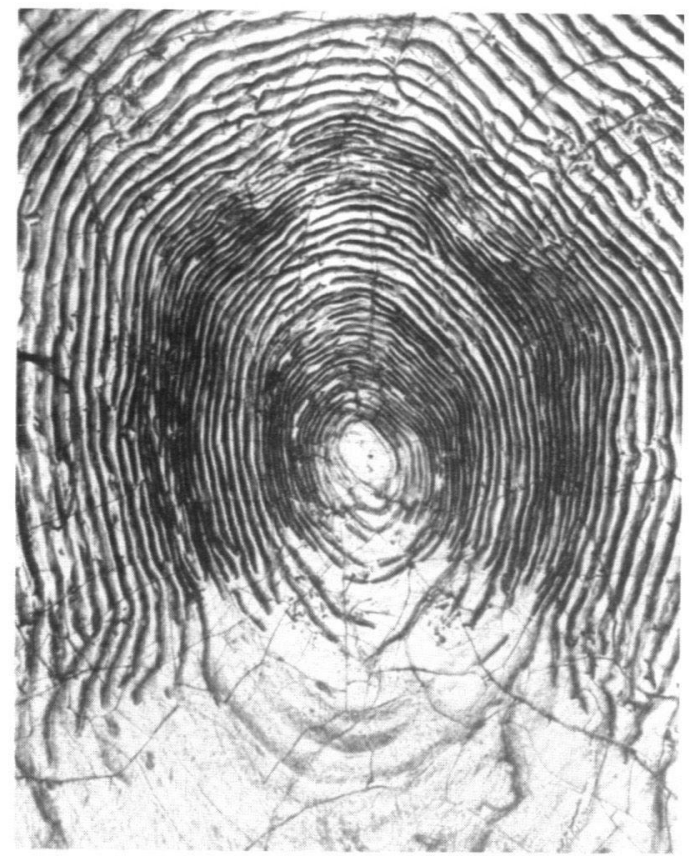

Figure 22 : Adulte, Allier, Centre, France, 13.04.80, ligne, 780, 2.

Figure 22 : Adult, Allier, Centre, France. 13.04.80, rod, 780, 2.

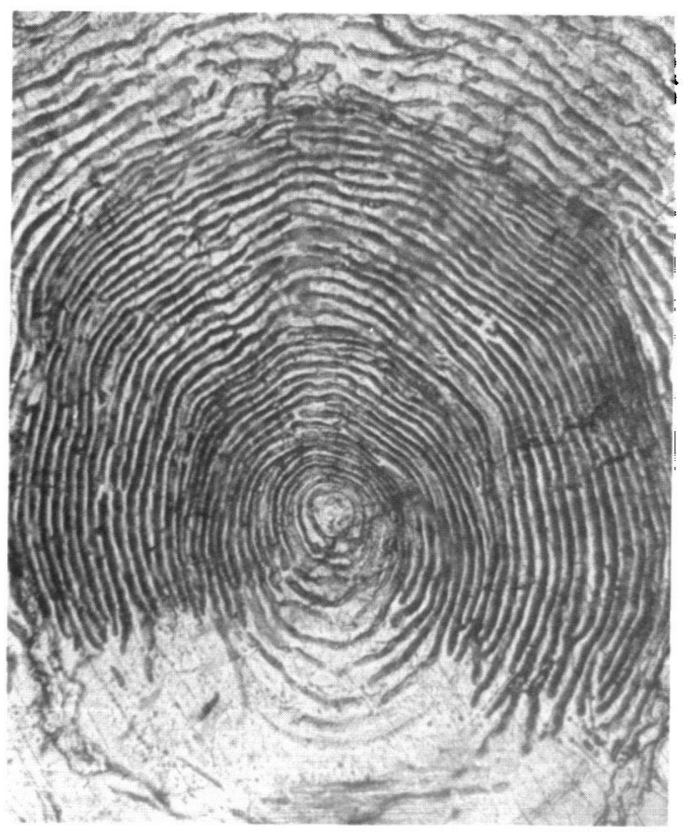

Figure 24 : Adulte. Gave d'Oloron. SudOuest, France, 23.04.77, ligne 920, 2.

Figure 24 : Adult, Gave d'Oloron, South West, France, 23.04.77, rod, 920, 2.

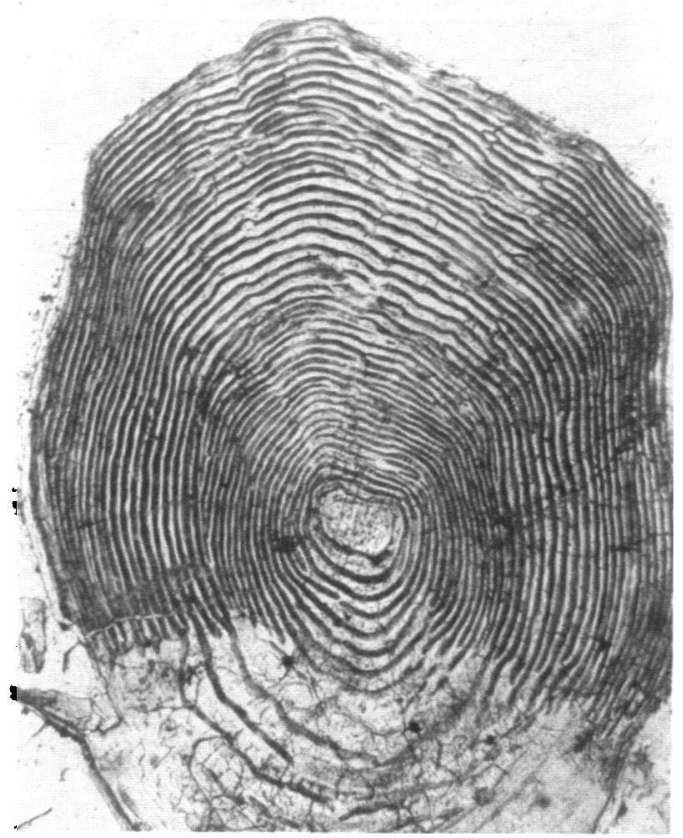

Figure 23 : Smolt, Gave d'Oloron, SudOuest, France, 24.03.78, piege de descente, $240,2^{+}$.

Figure 23 : Smolt, Gave d'Oloron. South West, France, 24.03.78. downstream trap, 240, $2^{+}$.

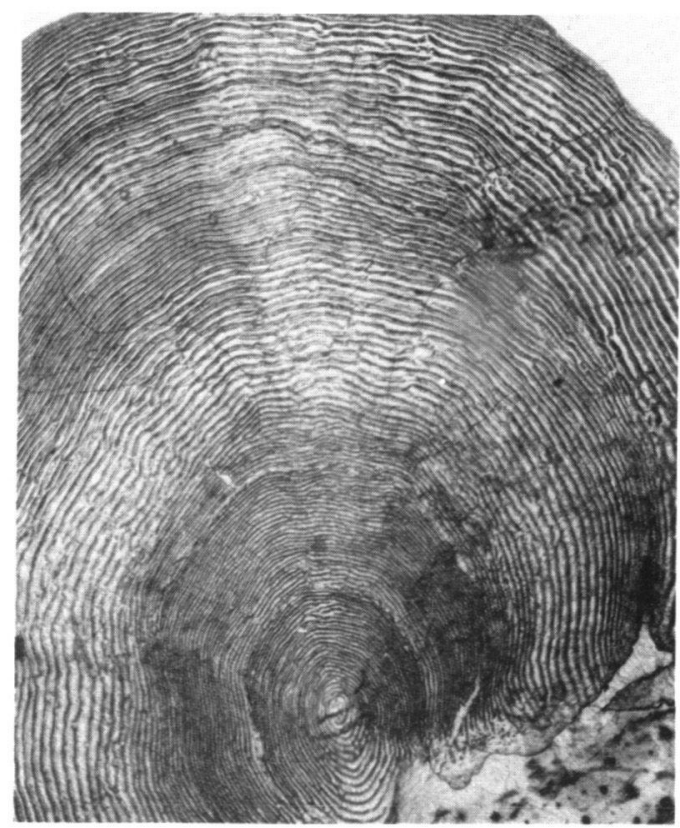

Fig. 24 bis : Adulte, Gave d'Oloron,

Sud-Ouest France, 8.06.82, ligne, 830,2 , très forte croissance en eau douce.

Figure 24 bis : Adult, Gave d'Oloron, South West, France, 8.06.82, rod, 830,2 , very big growth in freshwater. 


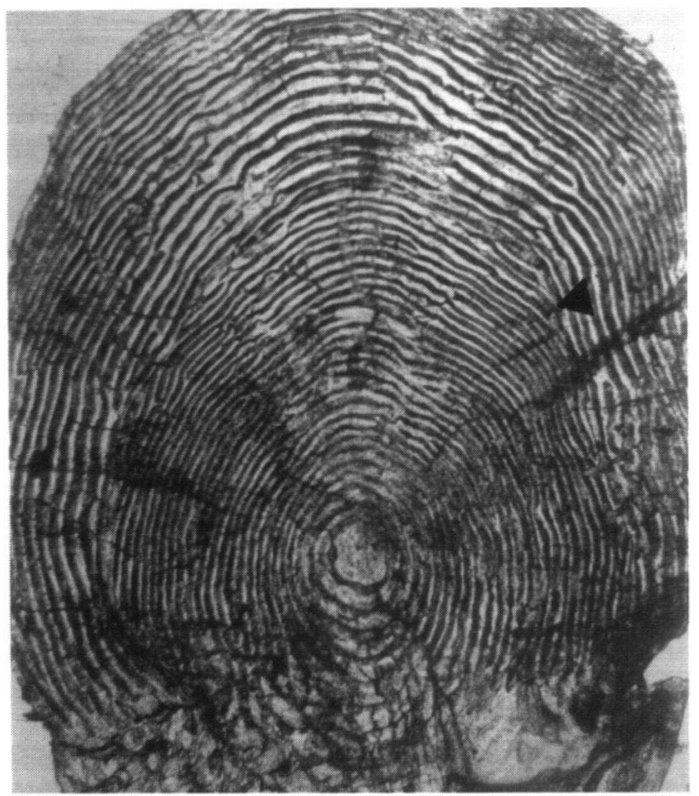

Figure 25 : Smolt, Gave d'Oloron, SudOuest, France, 21.03.78, piège de descente, 250, 2, marque de fraie lors du premier hiver $(\$)$.

Figure 25 : Smolt, Gave d'Oloron, South West, France, 21.03.78, downstream trap, 250, 2 , spawning mark at the first winter (D).

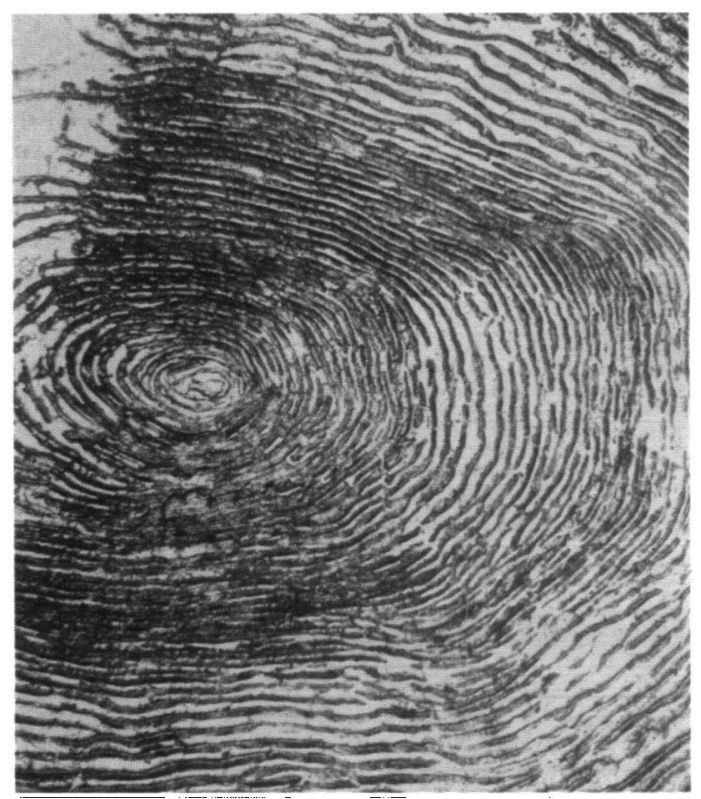

Figure 27 : Adulte, Esva, Asturies, Espagne, 30.04.84, ligne, 790 , $2^{+}$.

Figure 27 : Adult, Esva, Asturias, Spain, 30.04.84, rod, 790, $2^{+}$.

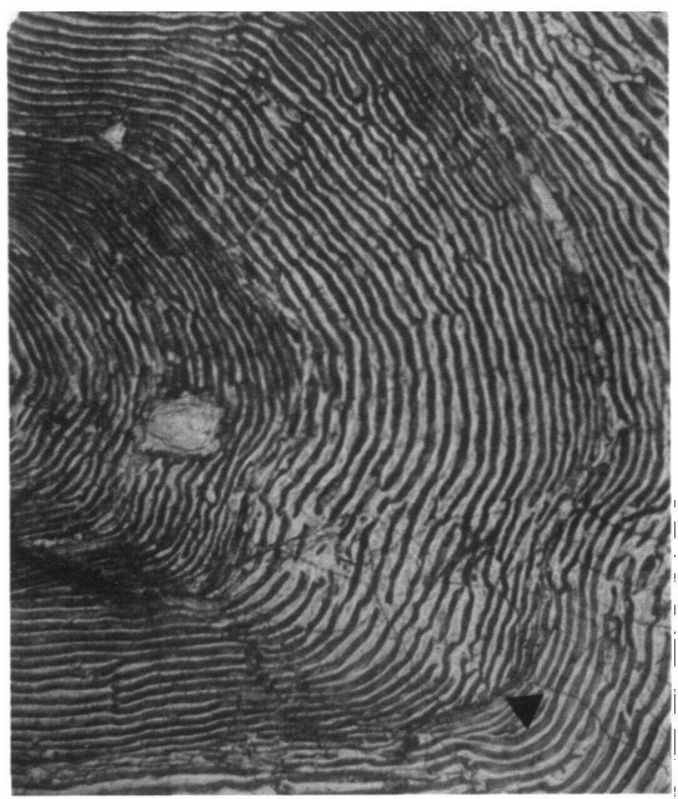

Figure 26 : Adulte, Nivelle, Sud-Ouest, France, 17.10.83, pêche électrique, 685, $\sigma, 2$, marque de fraie lors du deuxième hiver en eau douce ( $\downarrow)$.

Figure 26 : Adult, Nivelle, South West, France, 17.10.83, electrofishing, 685, $\varangle, 2$, spawning mark at the second freshwater winter $(\rightarrow)$.

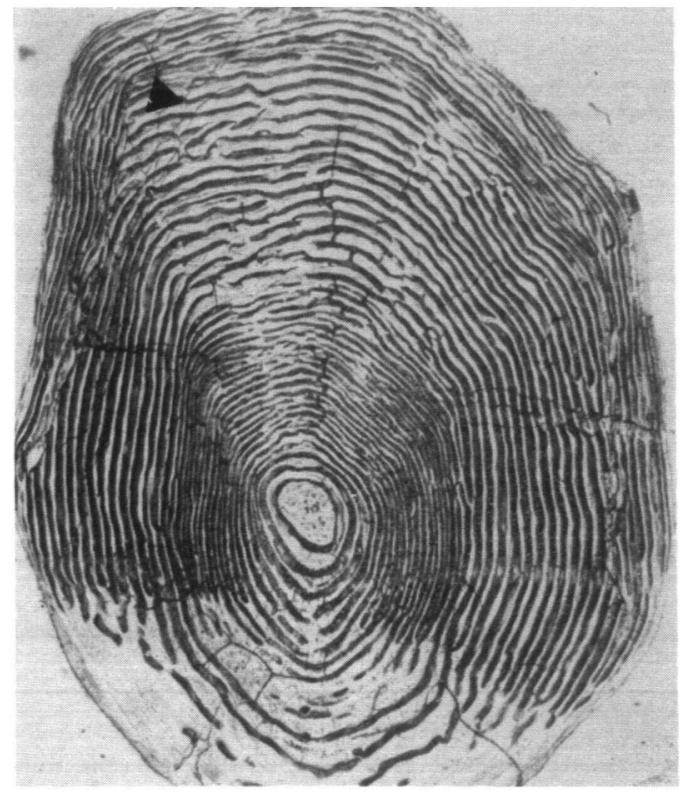

Figure 28 : Parr, Scorff, Bretagne, France, 26.09.78, pêche électrique, $210,2^{+}$, marque de fraie lors du deuxième hiver $(D)$.

Figure 28 : Parr, Scorff, Brittany, France, 26.09.78, electrofishing, 210. $2^{+}$, spawning mark at the second winter $(D)$. 


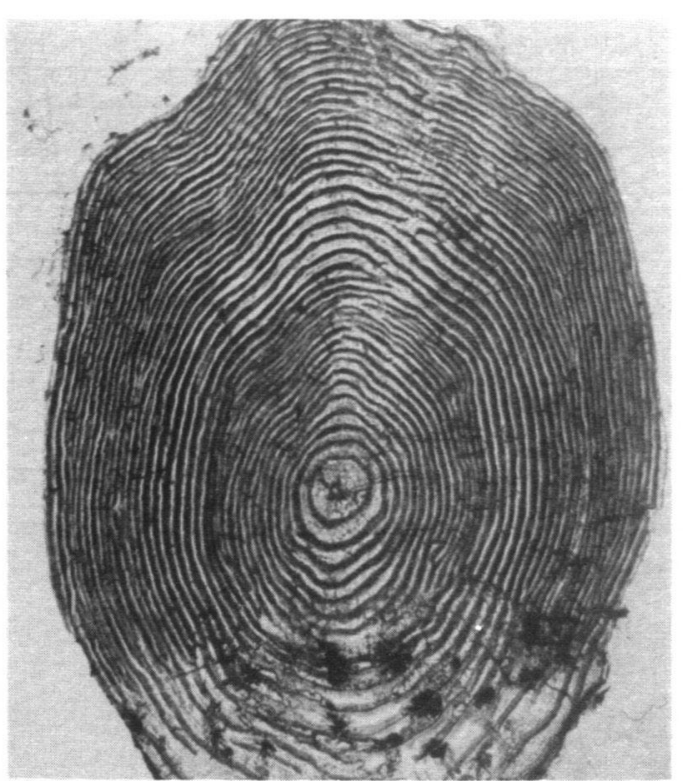

Figure 29 : Smolt, Gave d'Oloron, SudOuest, France, 24.03.78, piège de descente, 252, $3^{+}$.

Figure 29 : Smolt, Gave d'Oloron, South West, France, 24.03.78, downstream trap. 252, $3^{+}$.

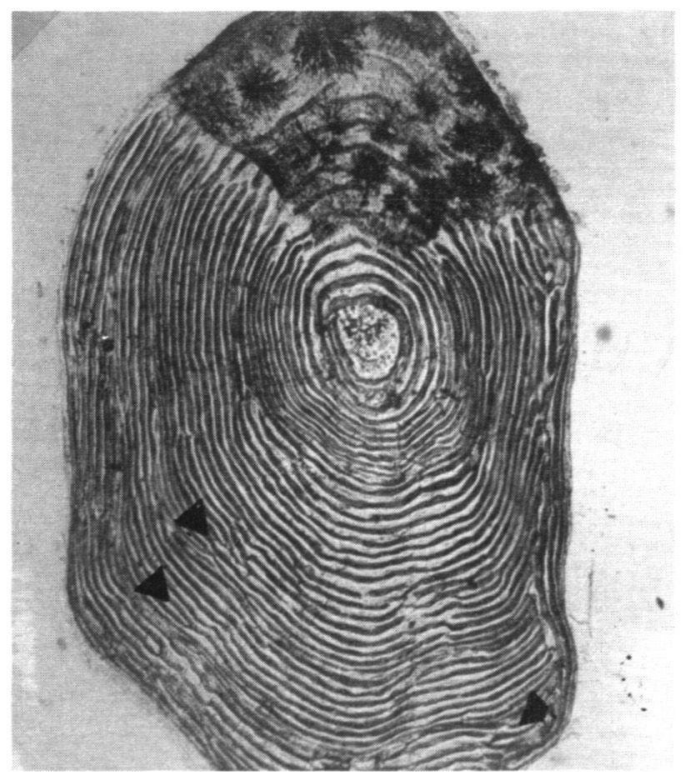

Figure 31 : Parr, Scorf, Bretagne, France, 30.04.74, pêche électrique. $211,4^{+}$, marques de fraie lors des deuxième, troisième et quatrième hivers $(\overrightarrow{)})$.

Figure 31 : Parr, Scorff, Brittany, France, 30.04.74, electrofishing, 211. $4^{+}$, spawning mark at the second, third and fourth freshwater winter ( $\$$ ).

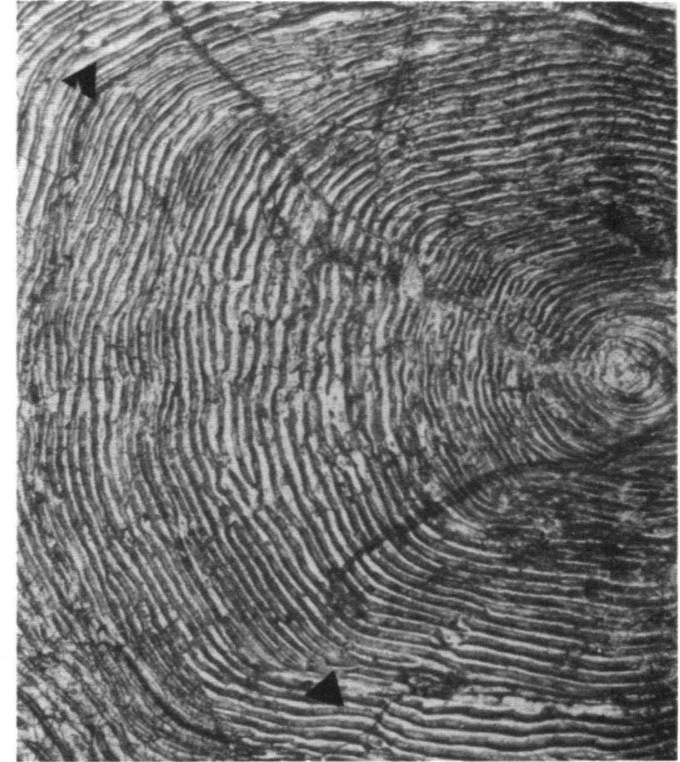

Figure 30 : Adulte, Léguer, Bretagne. France, 31.05.84, ligne. 820. 3. marques de fraie lors des deuxième et troisième hivers en eau douce $(>)$.

Figure 30 : Adult, Léguer, Brittany. France, 31.05.84, rod, 820, 3 ,

spawning mark at the second and third freshwater winter ( $\downarrow$ ).

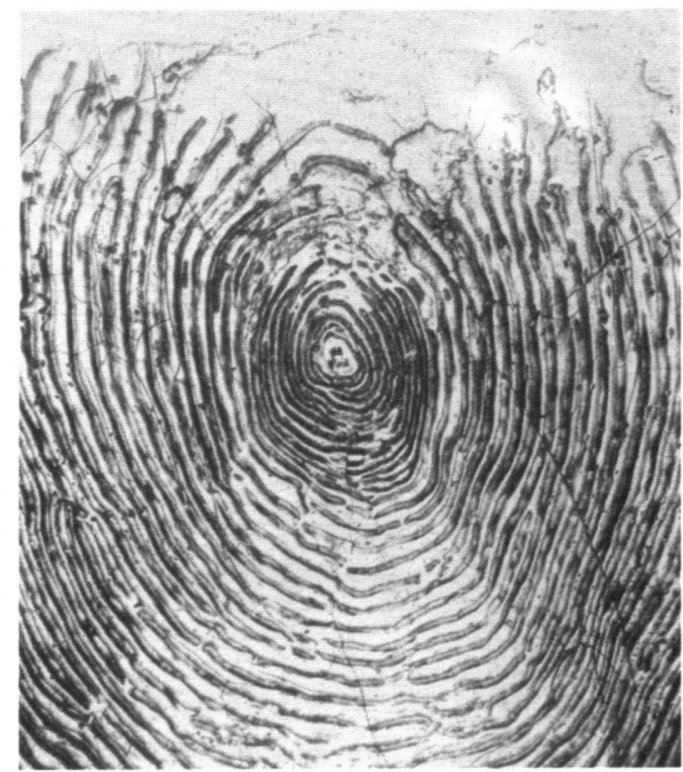

Figure 32 : Adulte, Eira, Norvège, pêche à la ligne, 1340, 2.

Figure 32 : Adult, Eira, Norway, rod 1340, 2. 


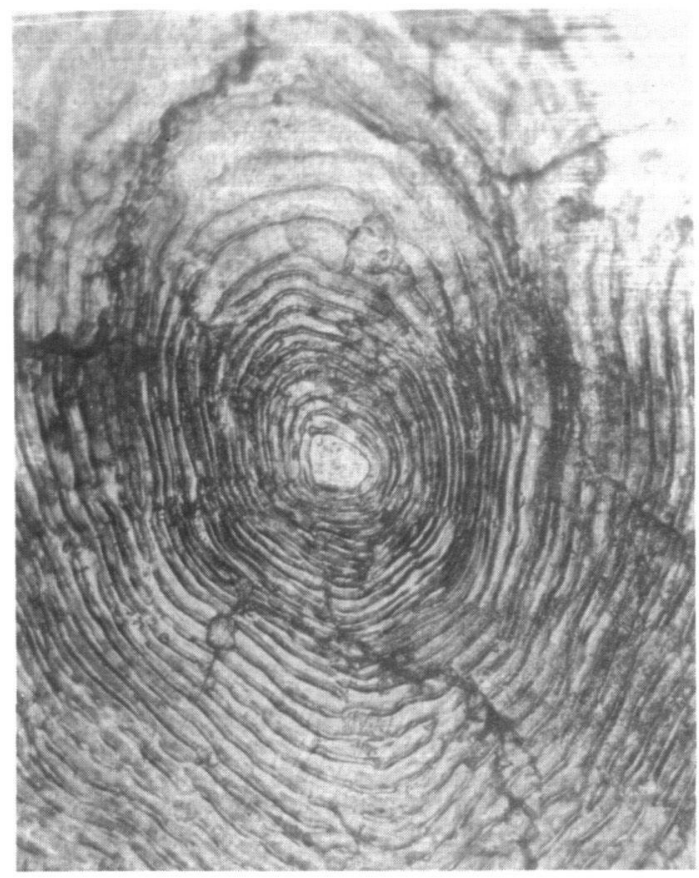

Figure 33 : Adulte, Tay, Ecosse, 23.04.82, ligne, 2.

Figure 33 : Adult, Tay, Scotland, 23.04.82, rod, 2 .

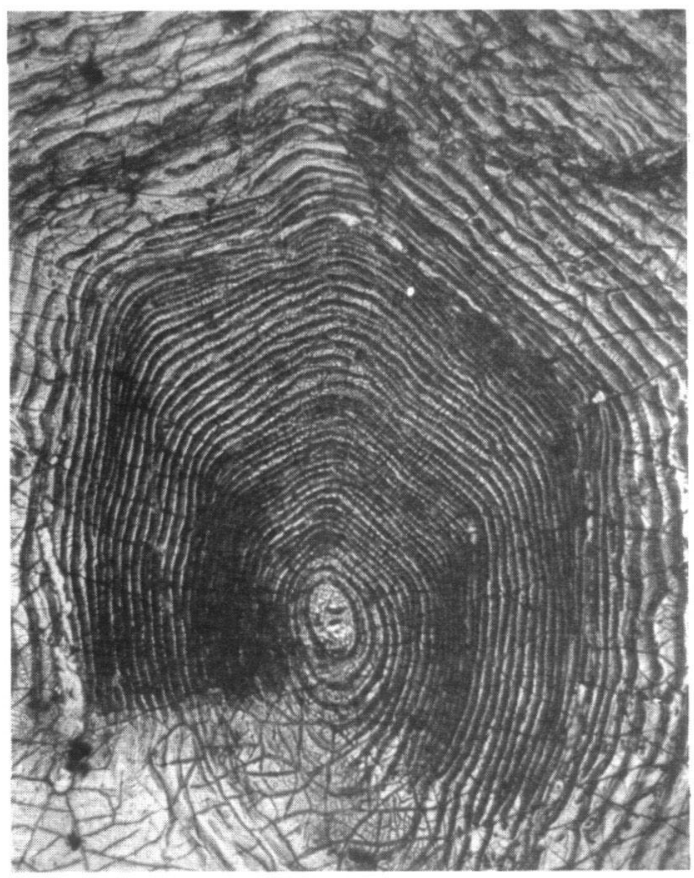

Figure 35 : Adulte, Exe, Sud-Ouest, Angleterre, 23.07.74, filet, 584, 2 .

Figure 35 : Adult, Exe, South West, England, 23.07.74, net, 584, $2^{*}$.

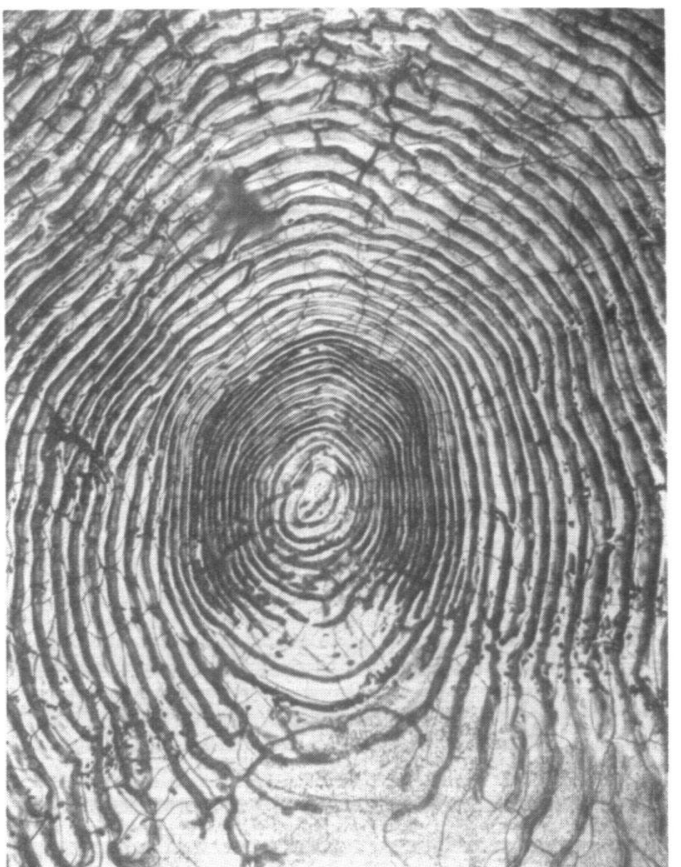

Figure 34 : Adulte, Irlande, 10.06.75, ligne, 860, 1

Figure 34 : Adult, Ireland, 10.06.75, rod, 860, 1 .

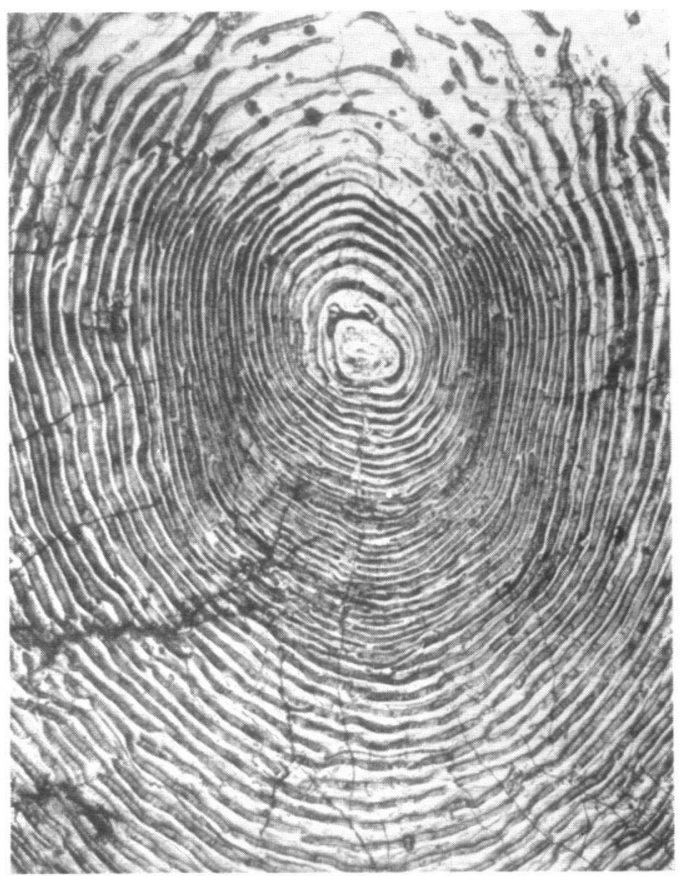

Figure 36 : Adulte, Penzé, Bretagne,

France, 20.03.80, ligne, 720 .

1 . présence de plusieurs rythmes de croissance.

Figure 36 : Adult, Penzé, Brittany, France, 20.03.80, rod, 720,1 , presence of several growth rhythms. 


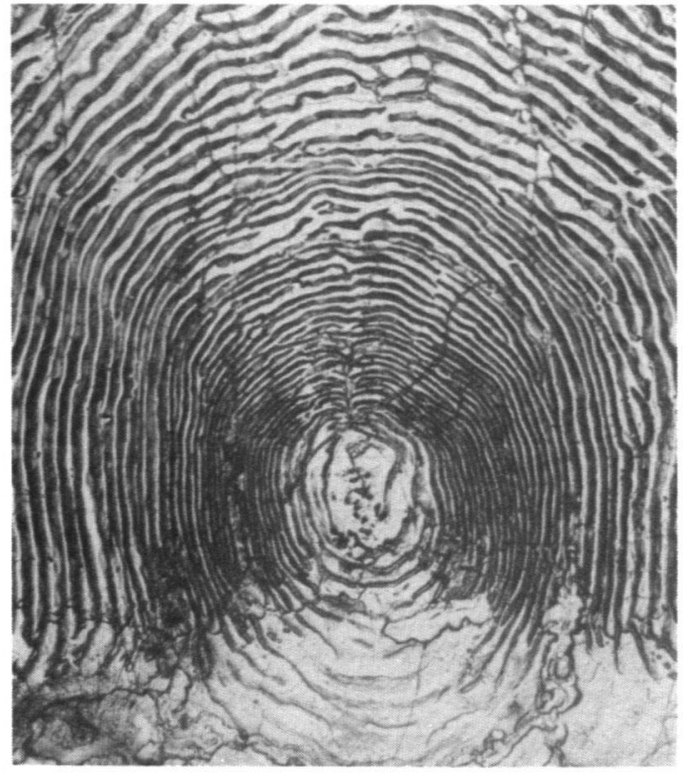

Figure 37 : Adulte, Allier, Centre, France, 2.04.80, ligne, $960,1^{+}$, présence de nombreux checks.

Figure 37 : Adult, Allier, Centre, France, 2.04.80, rod, $960,1^{+}$, presence of many checks.

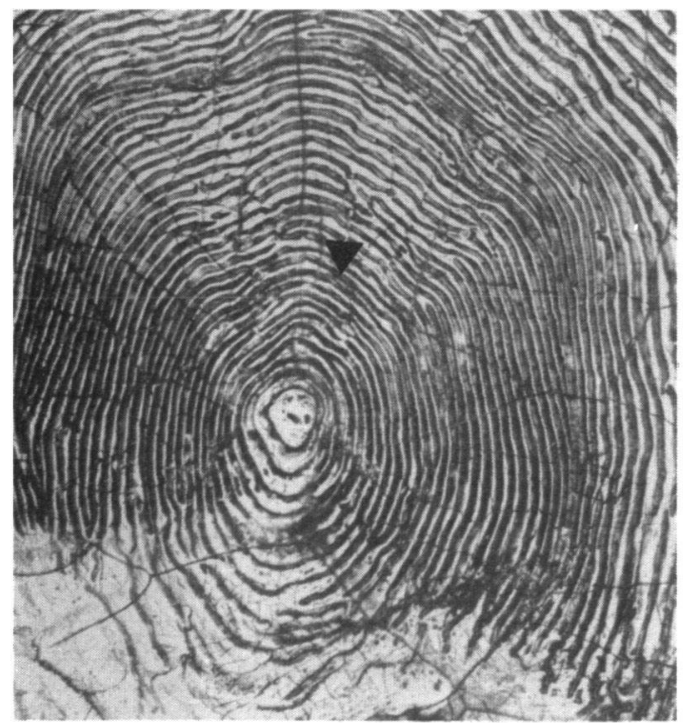

Figure 39 : Adulte, Odet, Bretagne, France, 22.03.75, ligne, 780, $1^{+}$ou $2^{+}$, présence d'un faux anneau au milieu de l'écaille ( et d'une possible marque de fraie sur le seul hiver visible.

Figure 39 : Adult, Odet, Brittany, France, 22.03.75, rod, $780,1^{\circ}$ or 2 , presence of a false annulus in middle of scale $(\rightarrow)$ and presence of a possible spawning mark at the only visible freshwater winter $(\downarrow)$.

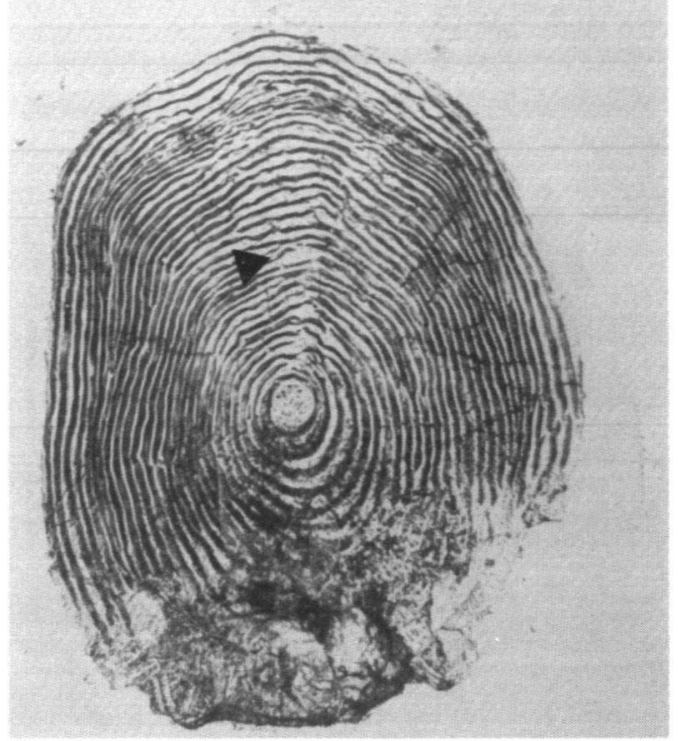

Figure 38 : Parr, Scorff, Bretagne, France, 28.05.84, pêche électrique, 134. $1^{+}$, présence d'un faux anneau au milieu de l'écaille (b).

Figure 38 : Parr, Scorff, Brittany, France, 28.05.84, electrofishing, 134, $1^{+}$. presence of a false annulus in middle of scale $(\downarrow)$.

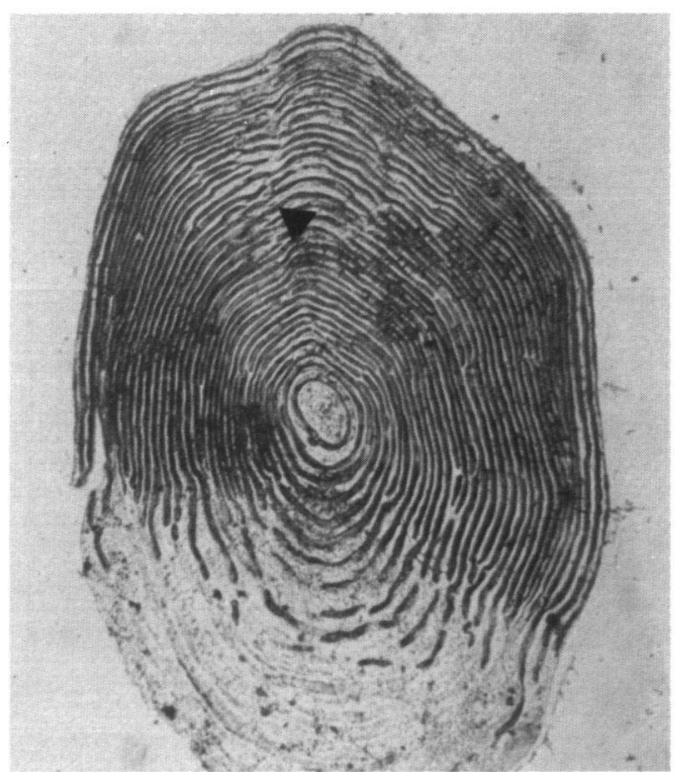

Figure 40 : Smolt, Gave d'Oloron, SudOuest, France, 14.04.78, piège de descente, 192, $1^{+}$, présence d'un faux anneau au milieu de l'écaille ( ).

Figure 40 : Smolt, Gave d'Oloron, South West, France, 14.04.78. downstream trap, $192,1^{+}$, presence of a false annulus in middle of scale $(\triangleright)$. 


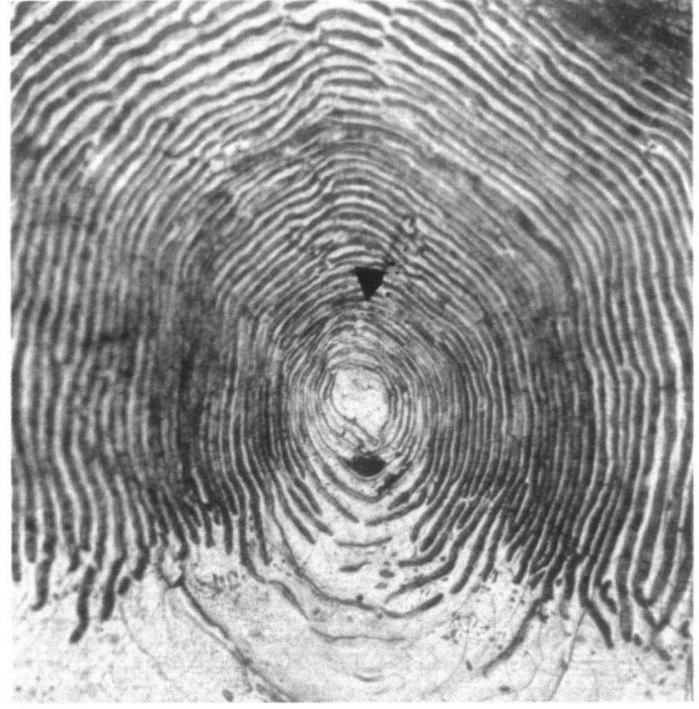

Figure 41 : Adulte, Gave d'Oloron, SudOuest. France, 27.04.80, ligne,

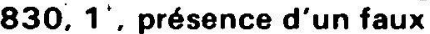
anneau au centre de l'écaille ( $\bullet$ ).

Figure 41 : Adult, Gave d'Oloron, South West, France, 27.04.80, rod, $830.1^{+}$, presence of a false annulus in middle of scale ( () ).

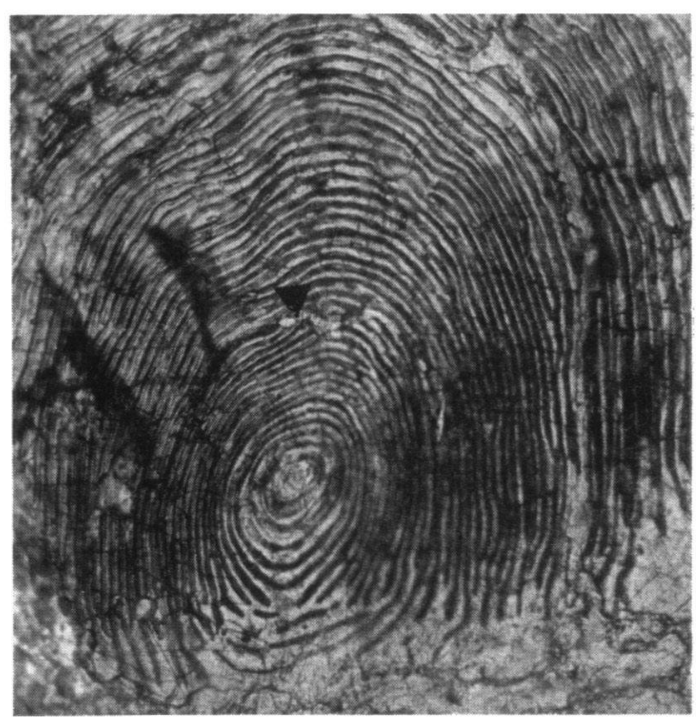

Figure 43 : Adulte, Allier, Centre, France, 22.05.81, ligne, $780,2^{+}$probable en raison de la taille de l'écaille, croissance continue avec la présence d'un faux anneau au milieu de l'écaille (D).

Figure 43 : Adult, Allier, Centre, France, 22.05 .81 , rod, $780,2^{+}$probably because of the scale size. continuous growth with a presence of a false annulus in middle of scale $(\downarrow)$.

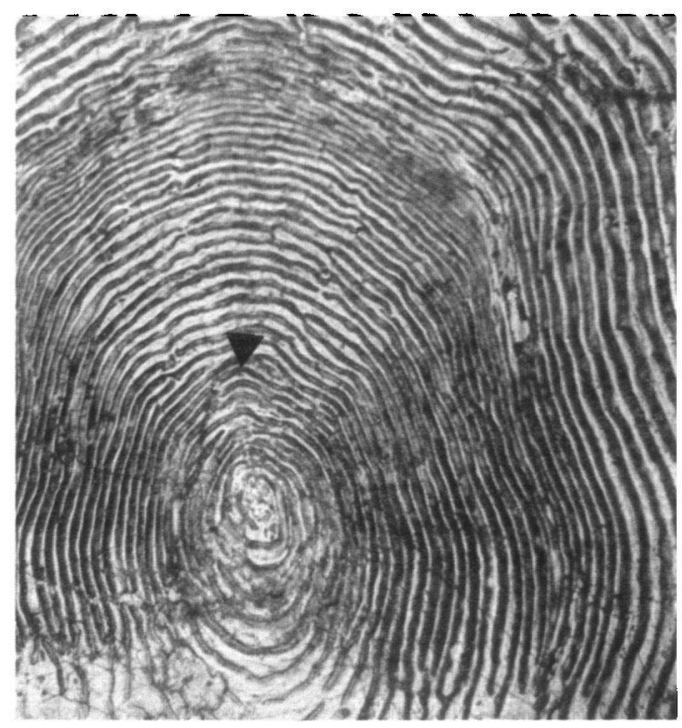

Figure 42 : Adulte, Nivelle, Sud-Ouest, France, 31.12.81, pêche électrique. $705,1^{+}$, présence d'un faux anneau au centre de l'écaille ( $)$.

Figure 42 : Adult, Nivelle, South West, France, 31.12.81, electrofishing, 705, $1^{+}$presence of a false annulus in middle of scale (D).

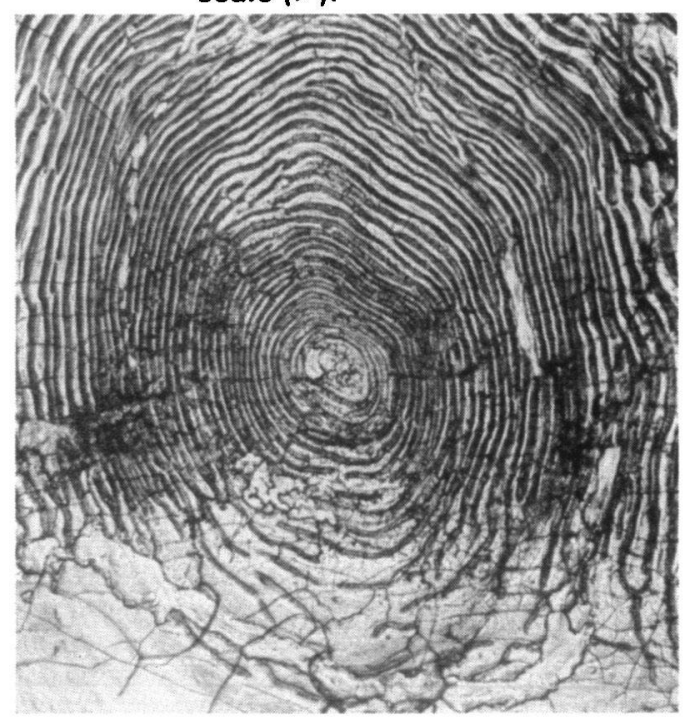

Figure 44 : Adulte, Ellé, Bretagne, France, 3.10.82, piège de montée, $660,2^{+}$probable. croissance continue en deuxième année. hiver non marqué, présence de checks.

Figure 44 : Adult, Ellé, Brittany, France, 3.10.82, upstream trap, 660 , $2^{+}$probably, with continuous growth during the second freshwater year, no visible winter, presence of checks. 


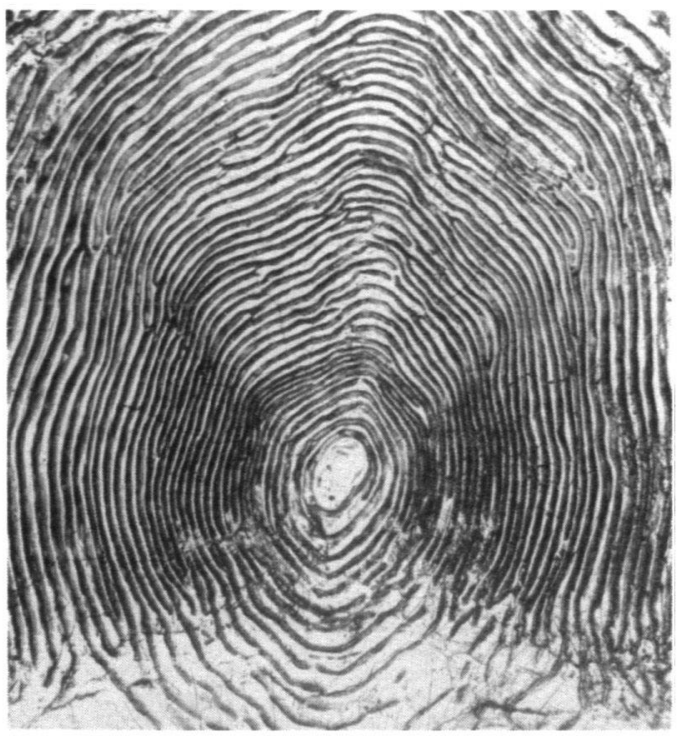

Figure 45 : Adulte, Steir, Bretagne, France, 11.05.75, ligne 790, $2^{+}$proba. ble, deuxième hiver en eau douce non marqué, deuxième année d'eau douce et phase smolt difficiles à distinguer.

Figure 45 : Adult, Steir, Brittany, France, 11.05.75, rod, $790,2^{+}$probably, no visible second freshwater winter, difficult to distinguish between second freshwater year and smolt stage.

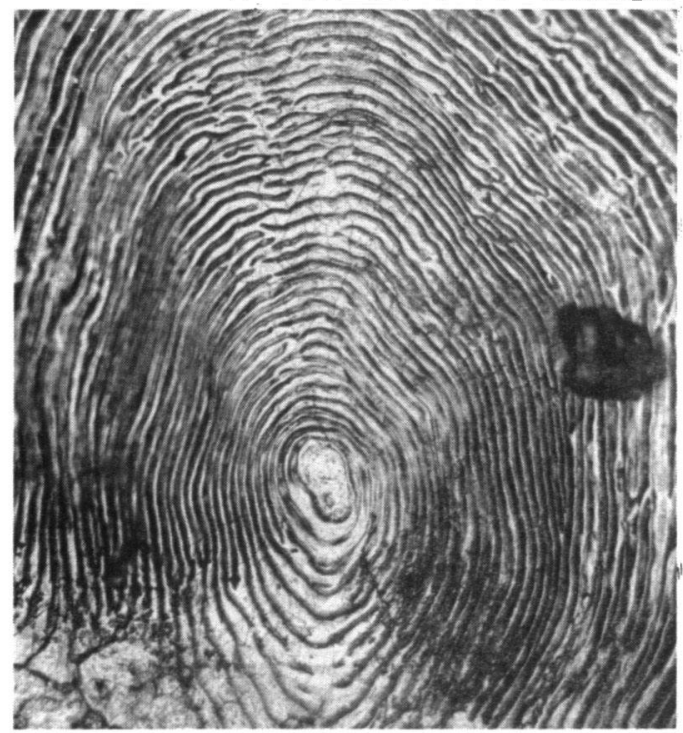

Figure 47 : Adulte, Gave d'Oloron, SudOuest, France, 15.04.81. ligne. $990,1^{+}$, croissance continue entre la zone d'eau douce et la phase smolt.

Figure 47 : Adult, Gave d'Oloron, South West, France, 15.04.81, rod, $990,1^{+}$, continuous growth between freshwater phase and smolt stage.

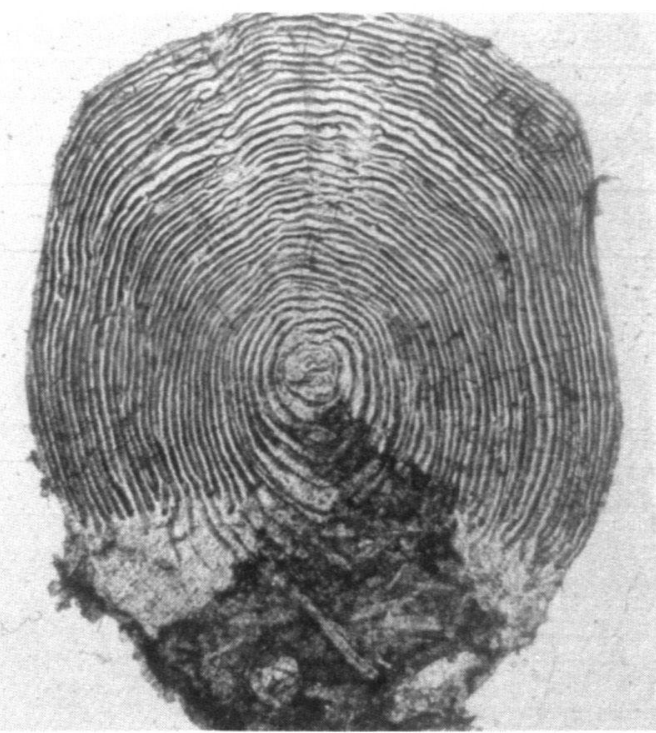

Figure 46 : Parr, Gave d'Oloron, SudOuest, France, 13.10.78, pêche électrique, 186, $1^{+}$probable en raison de la taille, croissance continue, absence d'hiver.

Figure 46 : Parr, Gave d'Oloron, South West, France, 13.10.78, electrofishing, 186, $1^{+}$probably on account of the length, continuous growth, no winter.

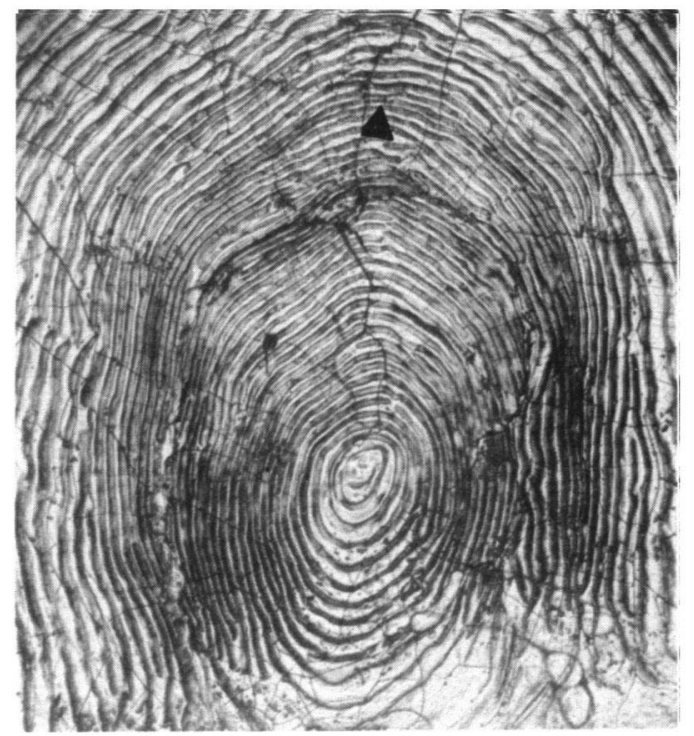

Figure 48 : Adulte, Scorff, Bretagne, France, 6.04.81, ligne, 745 , $1^{+}$, check au milieu de la phase smolt ( $(D)$.

Figure 48 : Adult, Scorff, Brittany. France, 6.04.81, rod, $745,1^{+}$, check in middle of smolt stage ( $)$. 


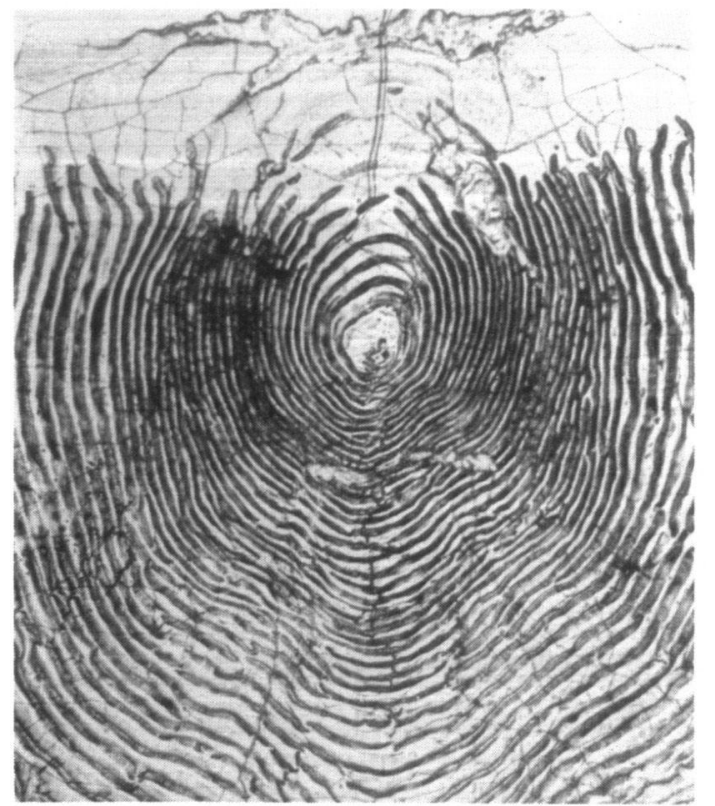

Figure 49 : Adulte, Allier, Centre, France, 3.05.80, ligne, 770, $2^{+}$probable, vraisembablement le resserrement correspond à un deuxième hiver en eau douce.

Figure 49 : Adult, Allier, Centre, France. 3.05.80, rod, $770,2^{+}$probably, likely the narrowing agrees with a second freshwater winter.

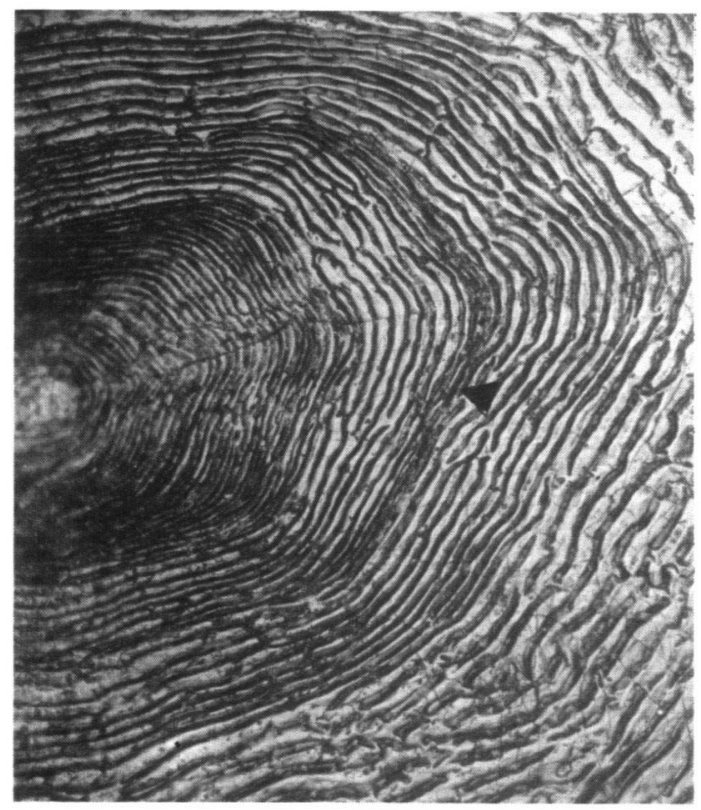

Figure 51 : Adulte. Loire. Centre, France, 26.05.84, filet, 820, $1^{+}$, présence d'un check en phase smolt ( $(-)$.

Figure 51 : Adult, Loire, Centre, France, 26.05.84, net, 820, $1^{+}$, presence of check in smolt stage (ヤ).

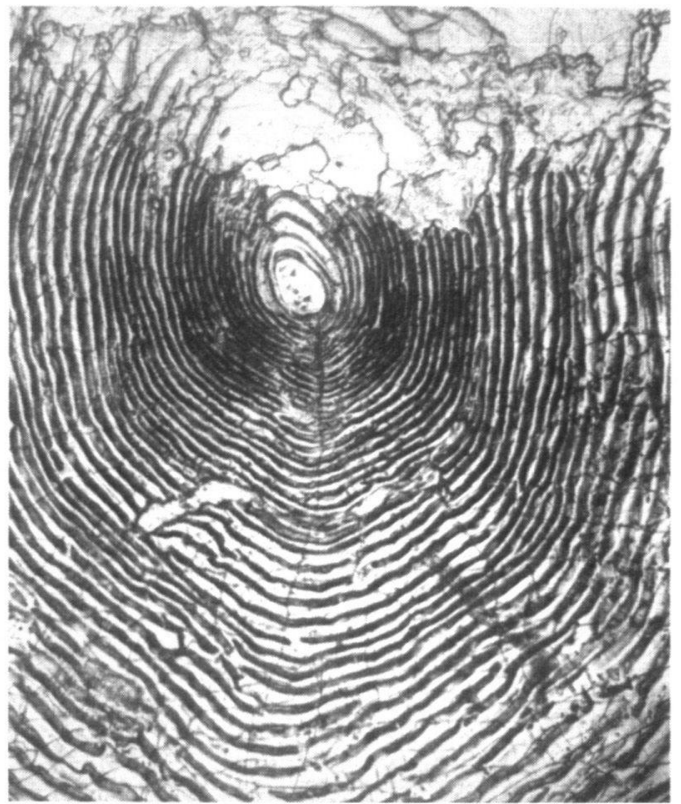

Figure 50 : Adulte, Allier, Centre, France, 5.04.80, ligne, $870,1^{+}$probable, deux rythmes de croissance dans la phase smolt.

Figure 50 : Adult, Allier, Centre, France. 5.04.80, rod, 870. $1^{+}$probably, two growth rhythms in smolt stage.

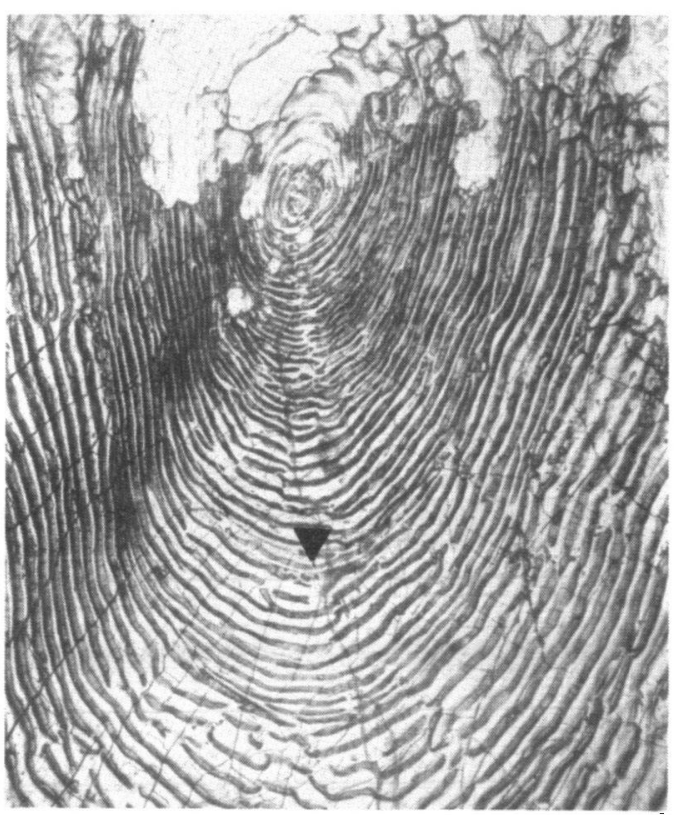

Figure 52 : Adulte, Allier, Centre, France, 9.04.80, ligne, 1000, $1^{+}$possible, check en phase smolt ( $\$$ ).

Figure 52 : Adult, Allier, Centre. France, 9.04.80, rod, $1000,1^{+}$possible, check in smolt stage ( 1 ). 


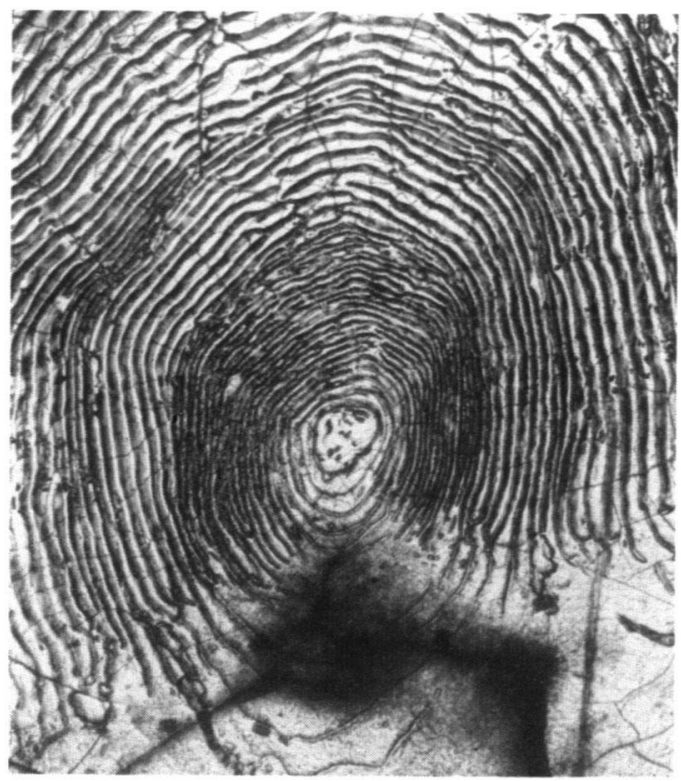

Figure 53 : Adulte, Allier, Centre, France. 12.04.80, ligne, $950,1^{+}$, check typique en fin de phase smolt.

Figure 53 : Adult, Allier, Centre, France, 12.04.80, rod, $950,1^{+}$, typical check at end of smolt stage.

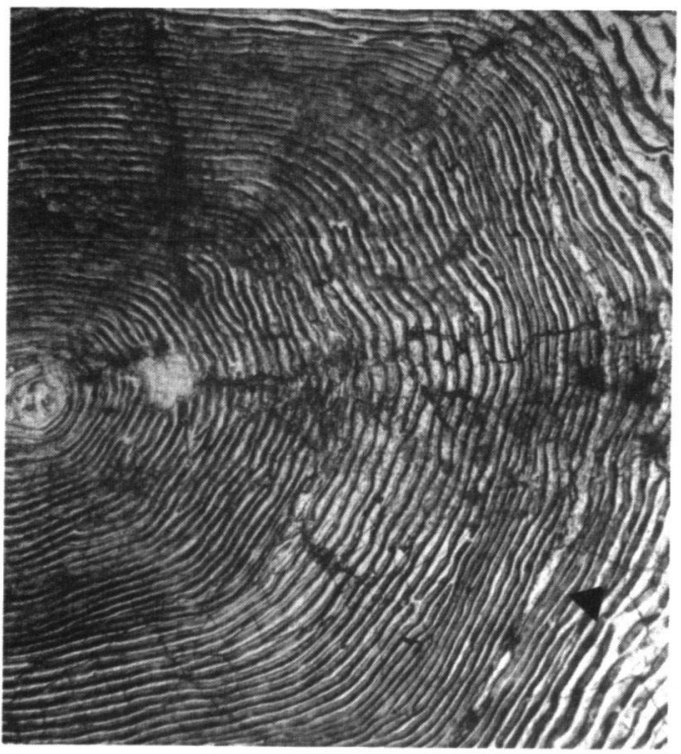

Figure 55 : Adulte, Gave d'Oloron, SudOuest, France, 23.06.80, ligne, 720, $2^{+}$probable, check en phase smolt $(\rightarrow)$ croissance trop faible pour une troisième année en eau douce.

Figure 55 : Adult, Gave d'Oloron, South West, France, 23.06.80, rod. $720,2^{+}$probably, check in smolt stage ( $\downarrow)$, too little growth for a third freshwater year.

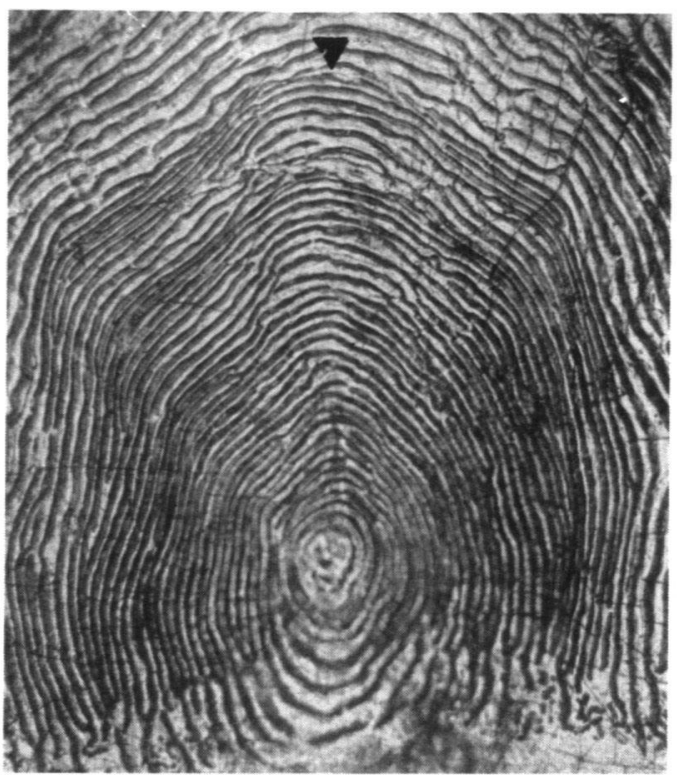

Figure 54 : Adulte, Gave d'Oloron, SudOuest, France, 12.04.77, ligne. $820,1^{+}$probable, check en fin de phase smolt ( $)$.

Figure 54 : Adult, Gave d'Oloron. South West, France, 12.04.77, rod, $820,1^{+}$probably, check at end of smolt stage $(D)$.

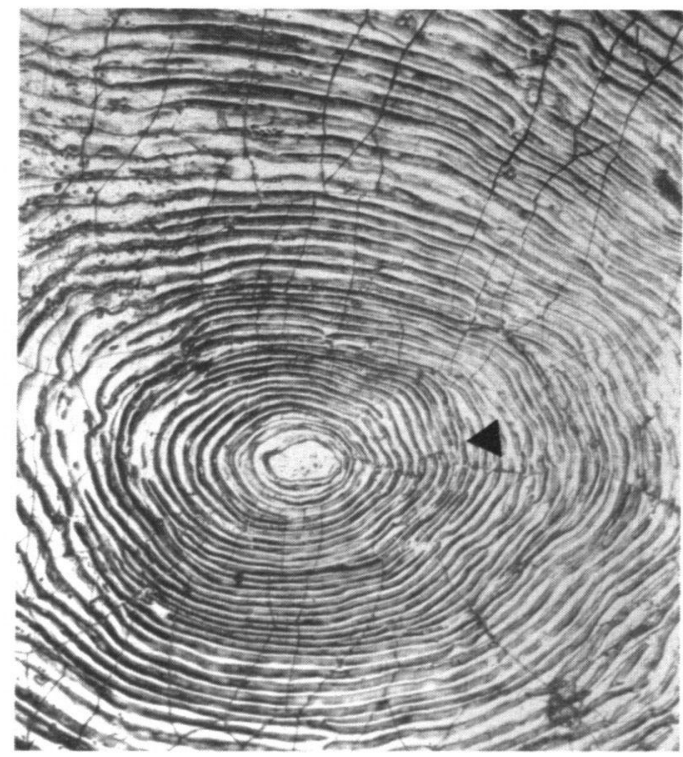

Figure 56 : Adulte, Elorn, Bretagne, France, 03.84, ligne, 770, $1^{+}$. poisson élevé en ruisseau pépinière marqué, existence d'un faux anneau $(D)$.

Figure 56 : Adult, Elorn, Brittany, France, 03.84, rod, $770,1^{+}$, tagged fish reared in nursery stream. presence of a false annulus ( $D$ ). 


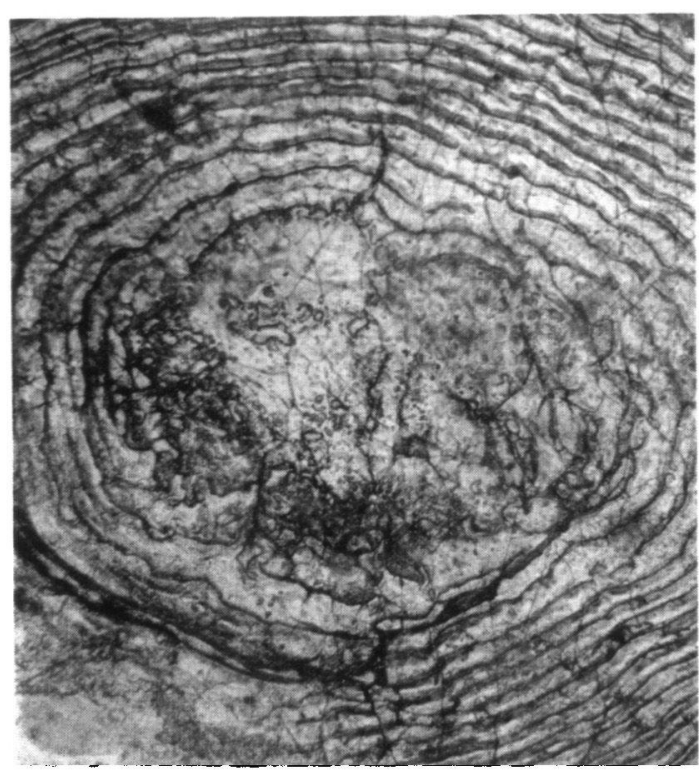

Figure 57 : Adulte, Nivelle, Sud-Ouest, France, 16.04.83, pêche électrique,,$+ 2^{+}$, regénération typique et fréquente de la partie eau douce chez les poissons élevés jusqu'au stade smolt.

Figure 57 : Adult, Nivelle, South West, France, 16.04.83, electrofishing.,$+ 2^{+}$, typical and frequent regeneration of the freshwater stage in fish reared up to smolt.

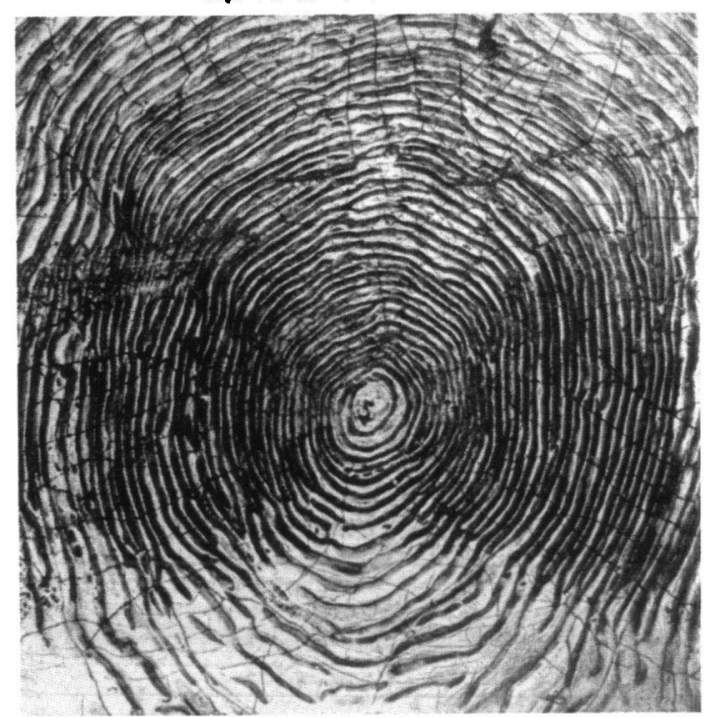

Figure 59 : Adulte, Elorn, Bretagne, France, 31.05.84, 727, $1^{+}$, poisson d'élevage marqué, différents rythmes de croissance semblable au cas de la figure 37.

Figure 59 : Adult, Elorn, Brittany. France, 31.05.84, 727, $1^{+}$, tagged rearing fish, different growth rhythms similar to Fig. 37 .

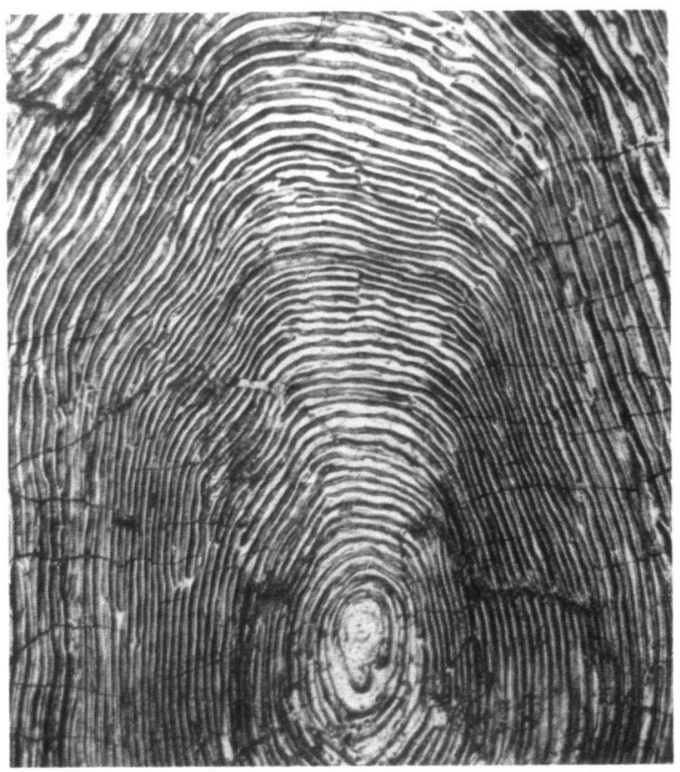

Figure 58 : Adulte, Elorn, Bretagne, France, 03.84, ligne, 754, $1^{+}$. poisson d'élevage marqué, croissance continue et presence de nombreux checks.

Figure 58 : Adult, Elorn, Brittany, France, 03.84, rod, 754, $1^{+}$, tagged rearing fish, continuous growth and presence of many checks.

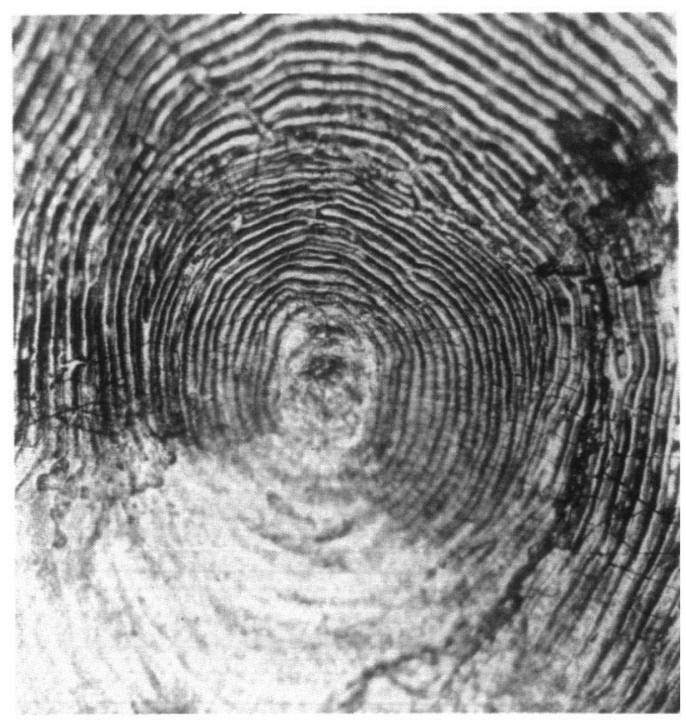

Figure 60 : Adulte, Allier, Centre, France, 28.02.76, ligne, $980,2^{+}$, poisson d'élevage marqué, détermination de l'âge impossible.

Figure 60 : Adult, Allier, Centre, France, 28.02.76, rod, $980,2^{+}$, tagged rearing fish, impossible age determination. 


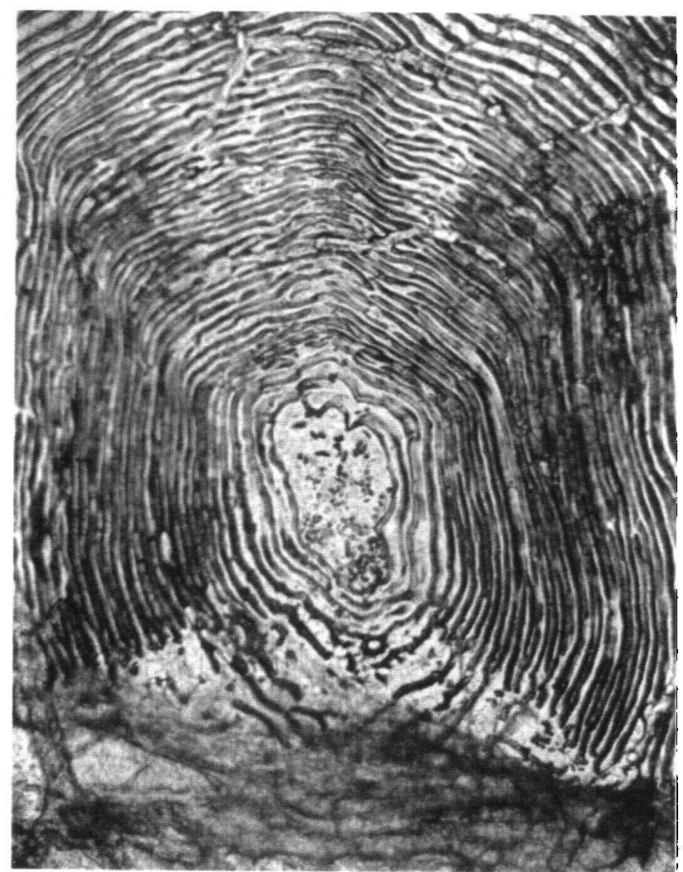

Figure 61 : Adulte, Nivelle, Sud-Ouest. France, 28.11.83, pêche électrique, 864, 2, poisson d'élevage marqué, détermination de l'âge impossible.

Figure 61 : Adult, Nivelle, South West. France, 28.11.83, electrofishing, 864, 2, tagged rearing fish, impossible age determination. 
LEGENDE DES FIGURES POUR LA PHASE MARINE

Rivière (affluent) - Région - Pays - Date et Technique de capture - Longueur totale (mm) - Sexe (supposé) - Age d'eau douce et de mer - Interprétations - Observations.

\section{LEGEND OF PLATES FOR SEAWATER STAGE}

River (Tributary) - Area - Country - Date and Technique of catch - Total length (mm) - Sex (supposed) - Freshwater and sea age - Interpretation - observations. 


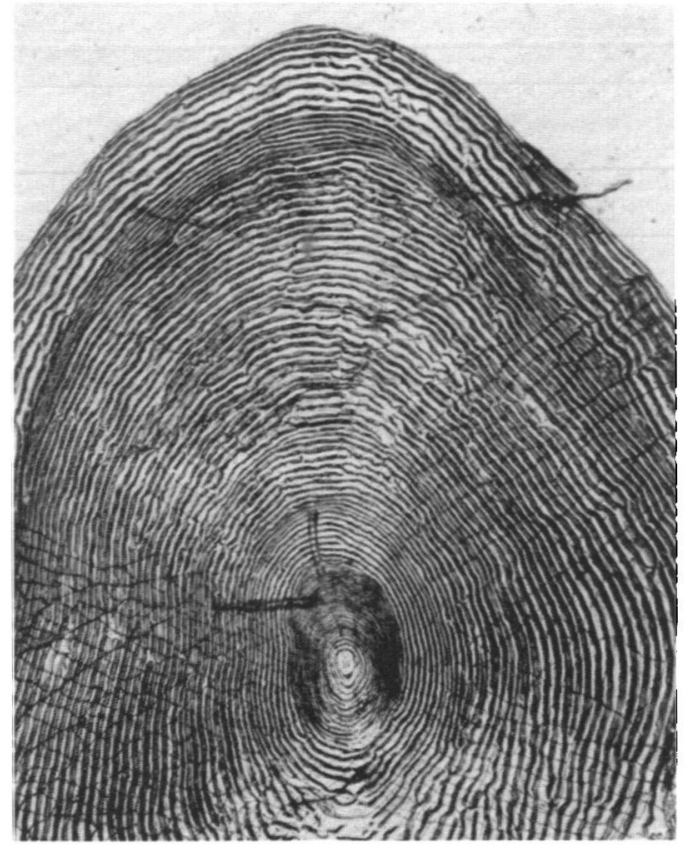

Figure 62 : Scorff. Bretagne, France, 11.06.81, ligne, 660, $]^{7}, 2.1^{+}$, castillon.

Figure 62 : Scorif, Brittany, France. 11.06.81, rod, $660,0^{\circ}, 2.1^{\circ}$. grilse.

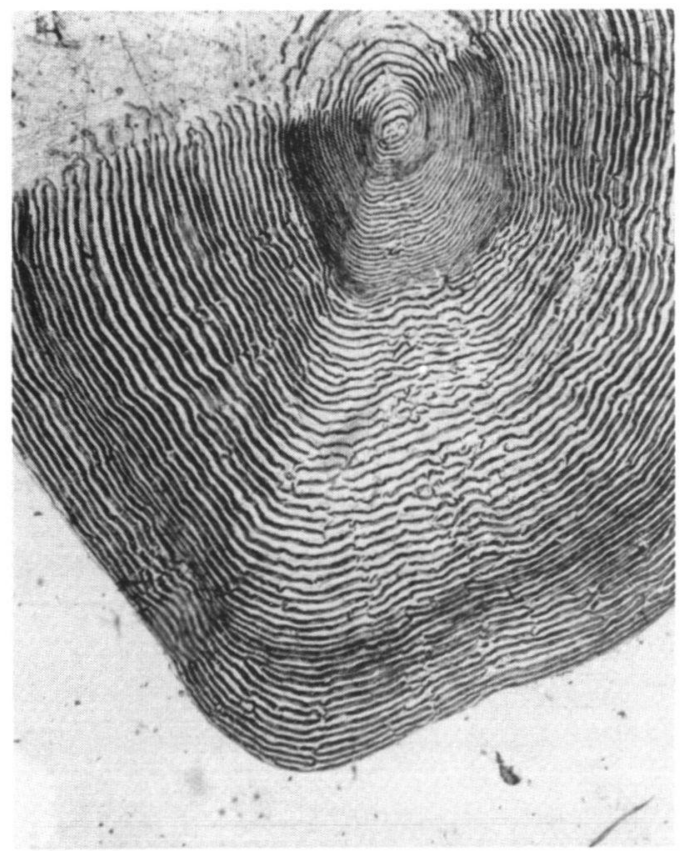

Figure 63 : Bresle, Nord, France, piège de montée, 770, $2.1^{+}$, castillon.

Figure 63 : Bresle, North, France, upstream trap. 770, 2.1 $1^{+}$, grilse.

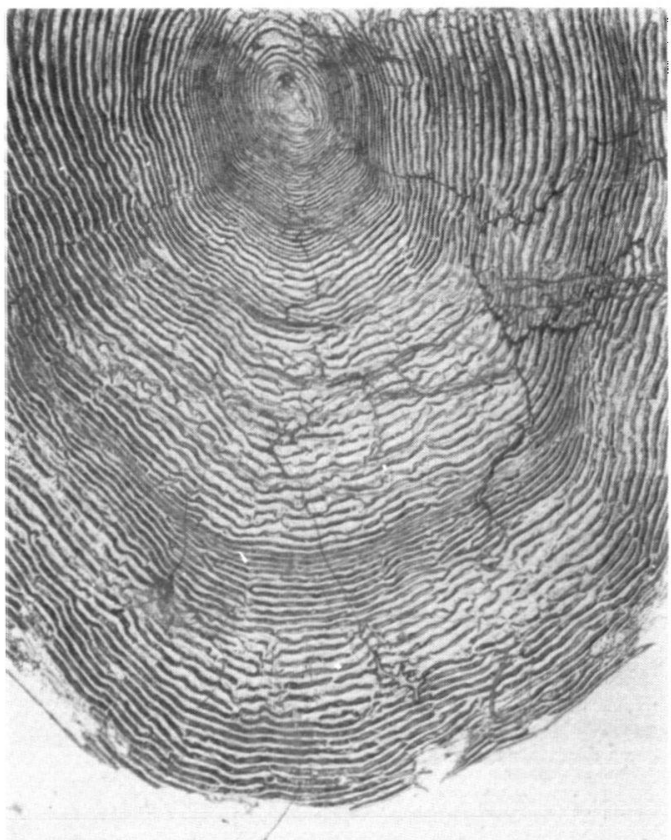

Figure 64 : Ellé, Bretagne, France, 3.10.82, piège de montée, $760,9,2.1^{+}$, castillon tardif, présence de poux de mer.

Figure 64 : Ellé, Brittany, France, 3.10.82, upstream trap, 760, $q, 2.1^{\circ}$. late grilse, presence of sea lice. 


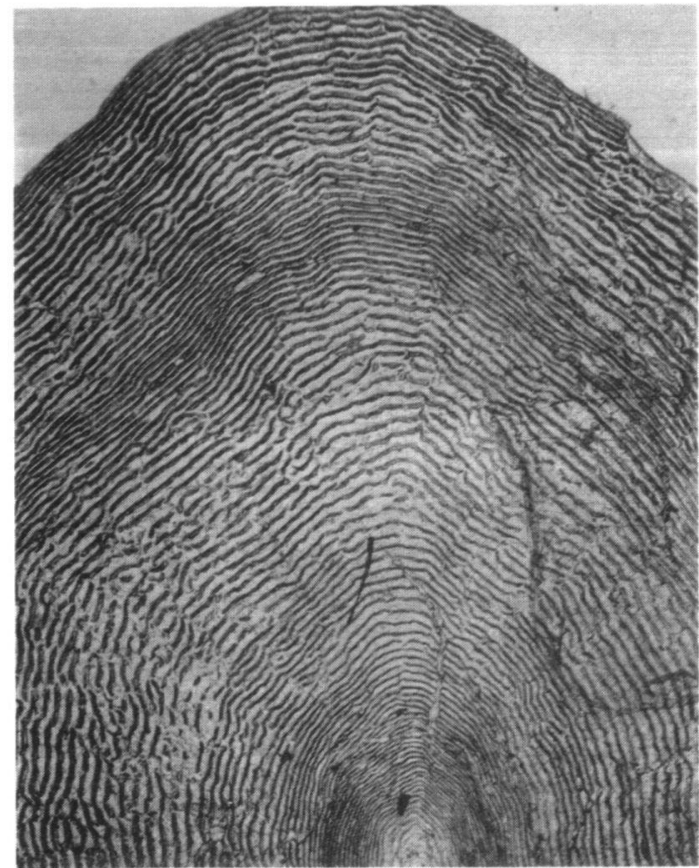

Figure 65 : Orne, Basse-Normandie, France, 20.11.84, piège de montée, 765, $\sigma^{7}, 1.1^{+}$, castillon tardif.

Figure 65 : Orne, Low Normandy, France, 20.11.84, upstream trap. 765 , $\sigma^{\top}, 1 \cdot 1^{+}$, late grilse.

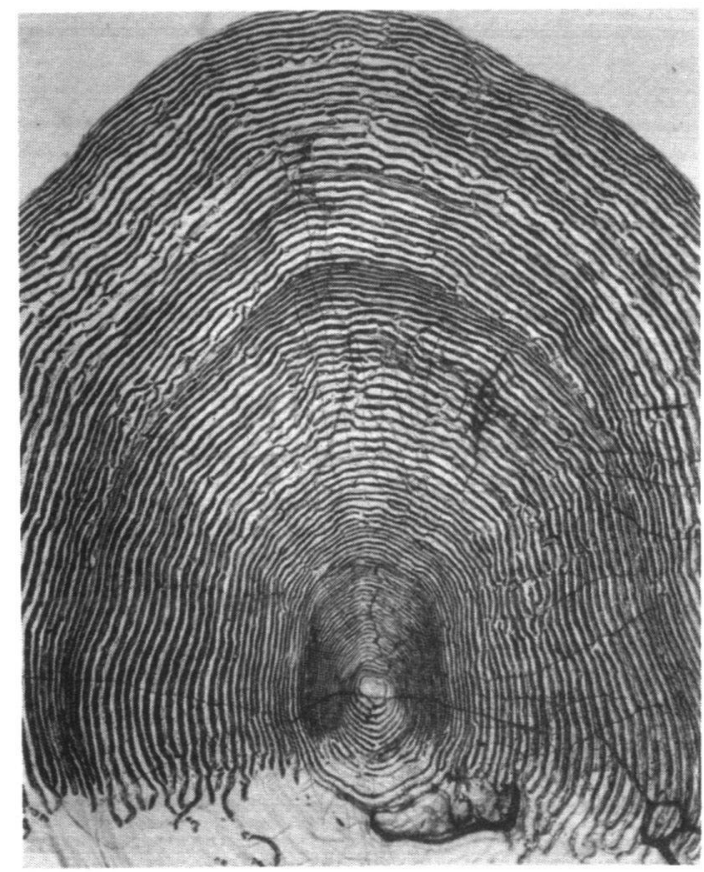

Figure 67 : Scorff, Bretagne, France, 6.04.81, ligne, 780, (J), 1.2. petit saumon de printemps.

Figure 67 : Scorft, Brittany, France, 6.04.81, rod, $780(\delta), 1.2$, small spring fish.

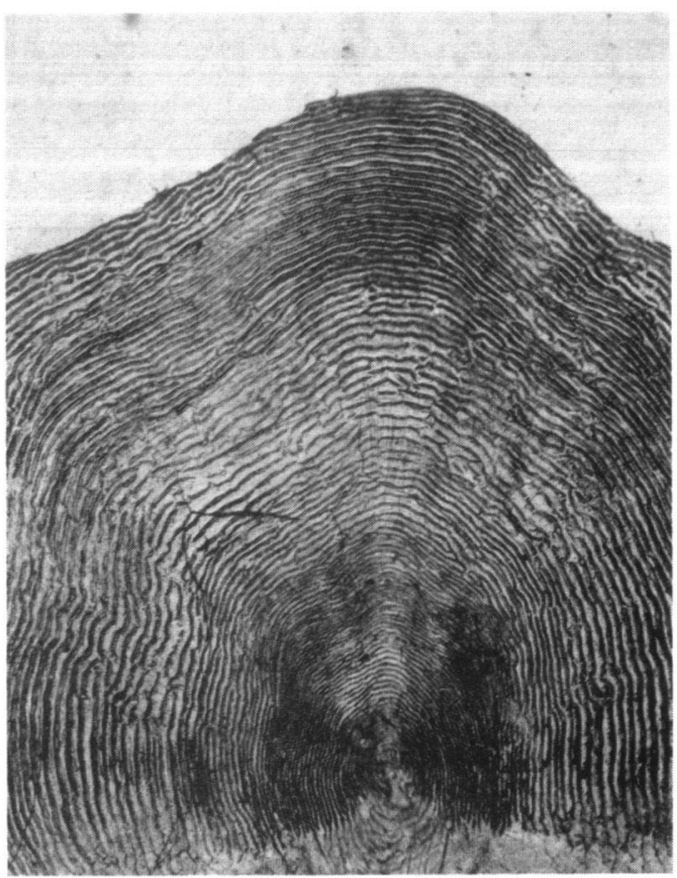

Figure 66 : Nivelle, Sud-Ouest, France, 26.08.81, pêche électrique, 830. $\sigma^{\prime}, 2.1^{\dagger}$, gros castillon tardif.

Figure 66 : Nivelle, South West, France, 26.08.81, electrofishing. 830 . ${ }^{3} \cdot \mathbf{2 . 1}^{+}$, big late grilse.

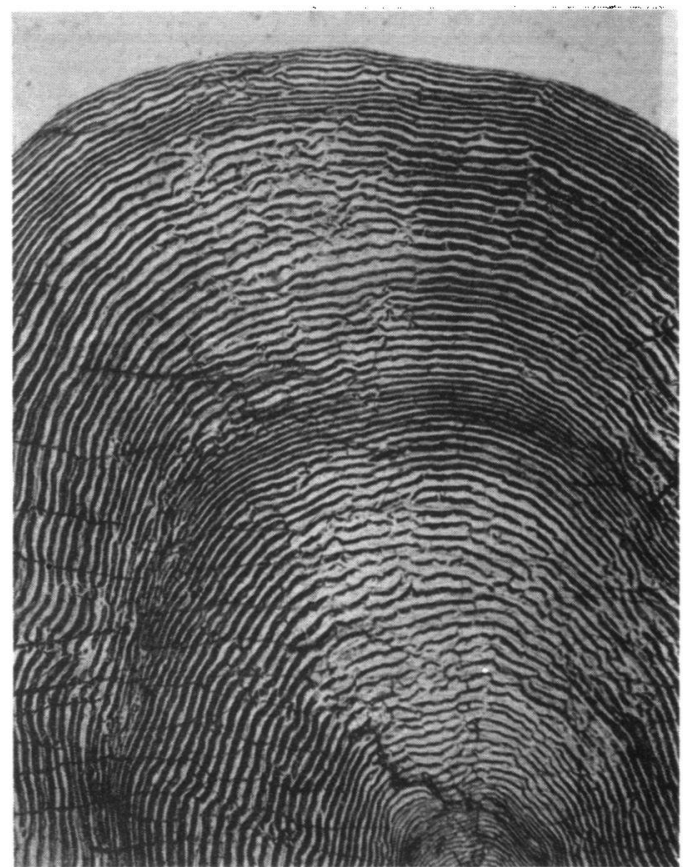

Figure 68 : Scorff, Bretagne. France, 11.04.81, ligne, 870. (Q), 1.2 , petit saumon d'été.

Figure 68 : Scorff, Brittany, France, 11.04.81, rod, $(q), 1.2^{+}$small summer fish. 


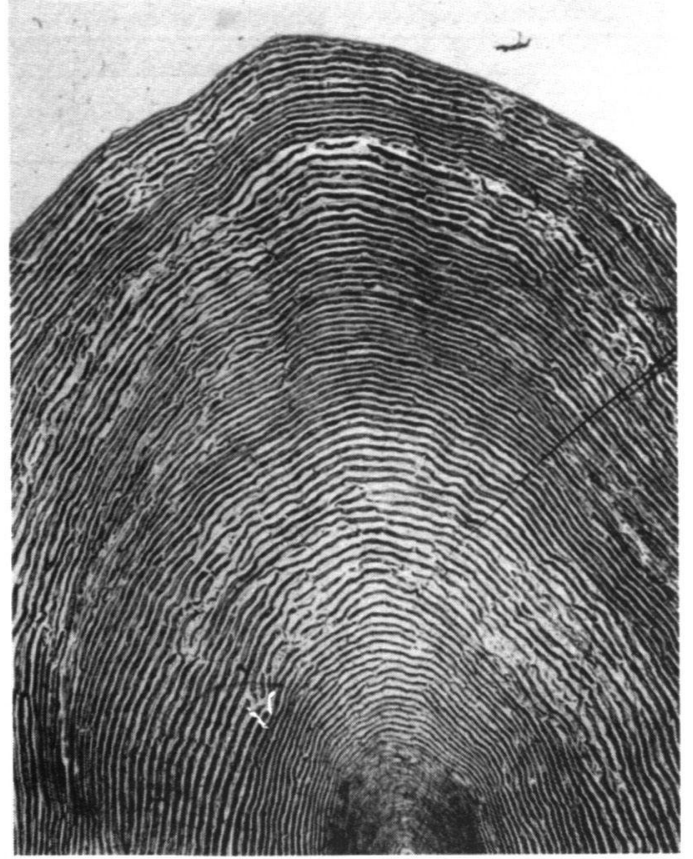

Figure 69 : Esva, Asturies, Espagne, 17.06.84, ligne, 830, 1.2 petit saumon d'été.

Figure 69 : Esva, Asturias, Spain, 17.06.84, rod, 830, $1.2^{+}$, small summer fish.

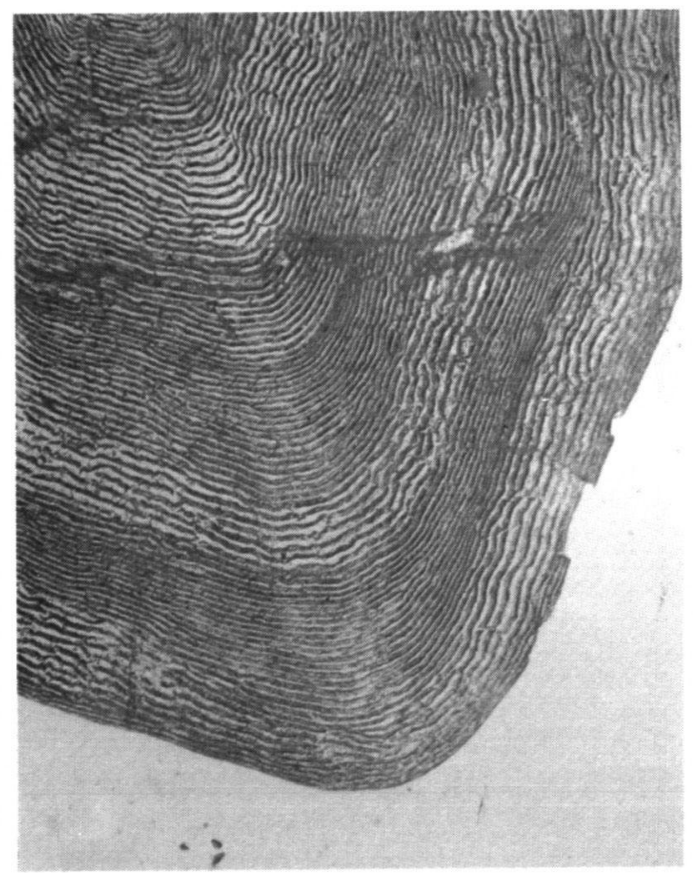

Figure 71 : Allier, Centre, France, 3.03.77, ligne, 970. (q), 1.3, grand saumon de printemps.

Figure 71 : Allier, Centre, France, 3.03.77, rod, 970, (१), 1.3, large spring salmon.

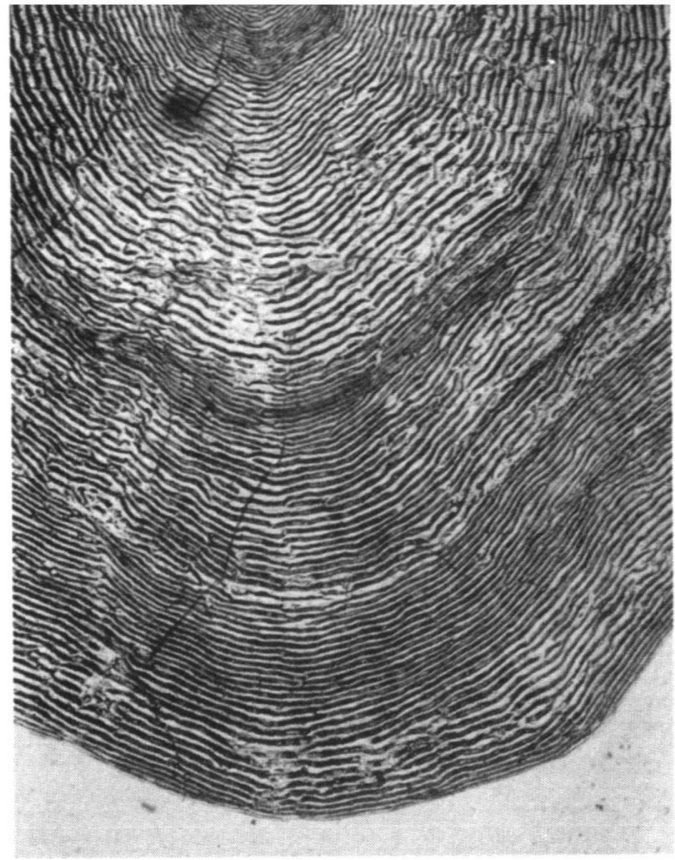

Figure 70 : Sée, Basse-Normandie, France, 4.04.81, ligne, 920 (). 1.3. grand saumon de printemps.

Figure 70 : Sée, Low Normandy, France, 4.04.81, rod, 920, (१). 1.3, large spring salmon.

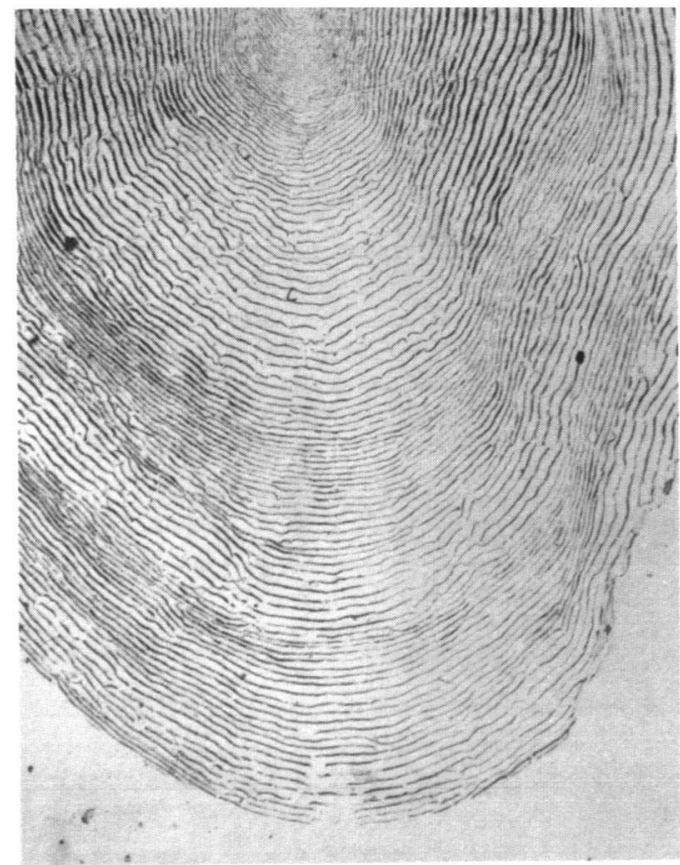

Figure 72 : Garonne, Sud-Ouest, France. 17.05.84, filet, 880, 1.3, grand saumon de printemps.

Figure 72 : Garonne, South West, France, 17.05 .84 , net, $880,1.3$, large spring salmon. 


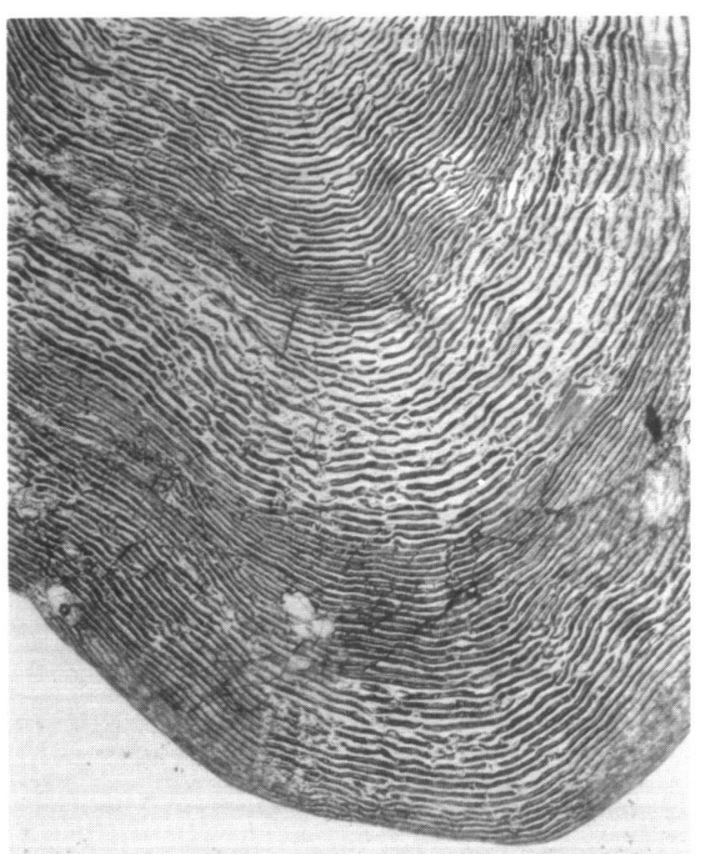

Figure 73 : Gave d'Oloron, Sud-Ouest, France, 15.04.81, ligne, 990 (१). 1.3, grand saumon de printemps.

Figure 73 : Gave d'Oloron, South West, France, 15.04.81, rod, 990 ( $)$ ), 1.3, large spring salmon.

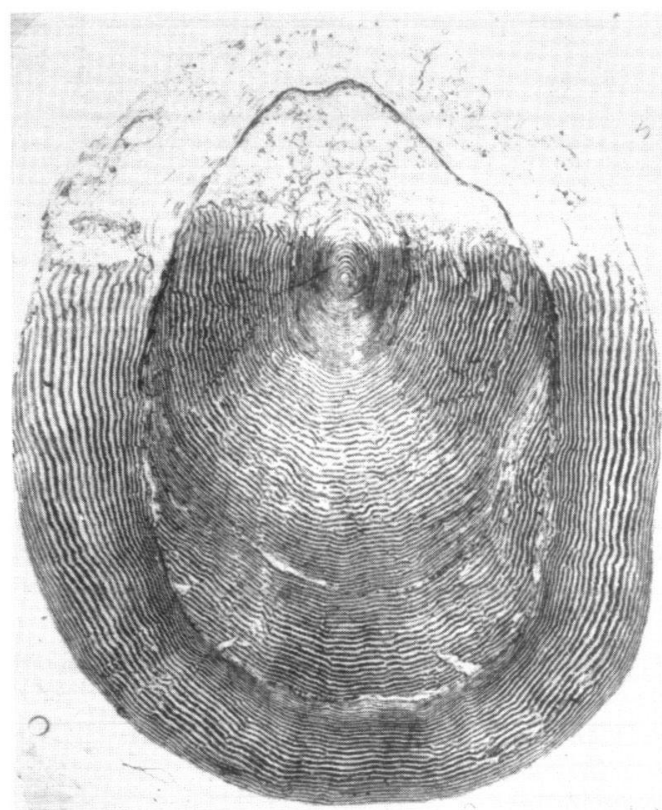

Figure 75 : Elorn, Bretagne, France, 07.75, ligne, 930, (1), 3, poisson de 2ème remontée.

Figure 75 : Elorn, Brittany, France, 07.75. rod, 930 . (\&), previous spawner. (one spawning).

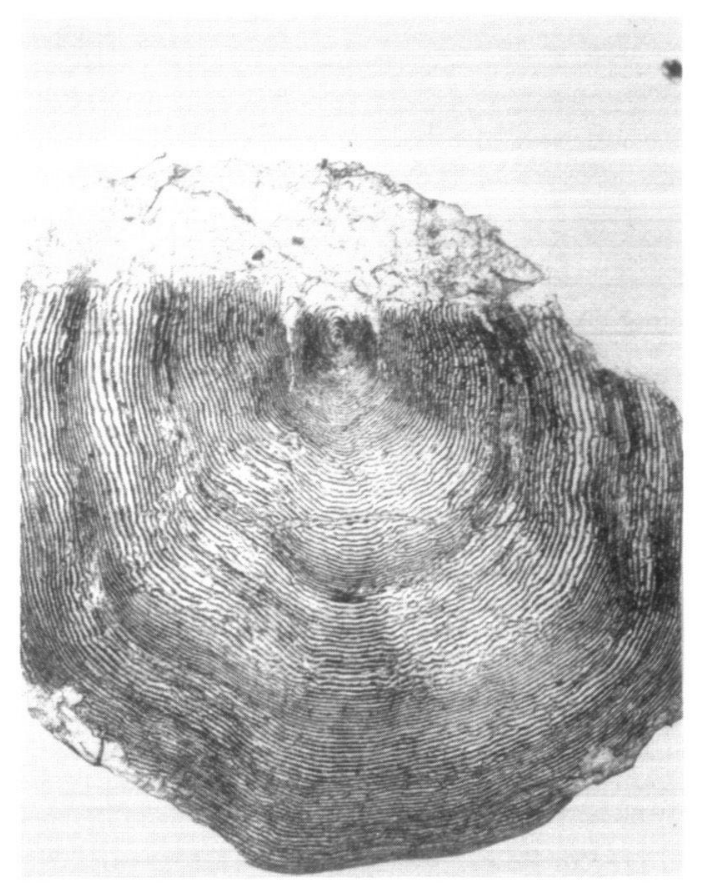

Figure 74 : Allier, Centre, France, 2.04.74, ligne, 1200, 1.4, très grand saumon de printemps.

Figure 74 : Allier, Centre, France, 2.04.74. rod, 1 200, 1.4, very large spring salmon.

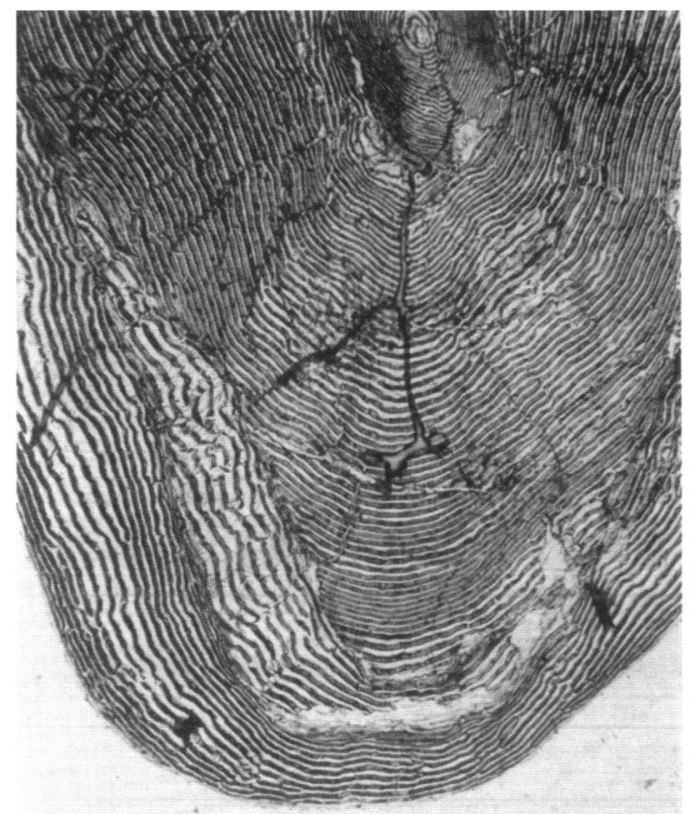

Figure 76 : Scorff, Bretagne, France, 7.03.81, ligne, 990,,+ 1 , poisson de 3ème remontée.

Figure 76 : Scorff, Brittany, France, 7.03.81, rod, 990, ?. 1, previous spawner (two spawning). 


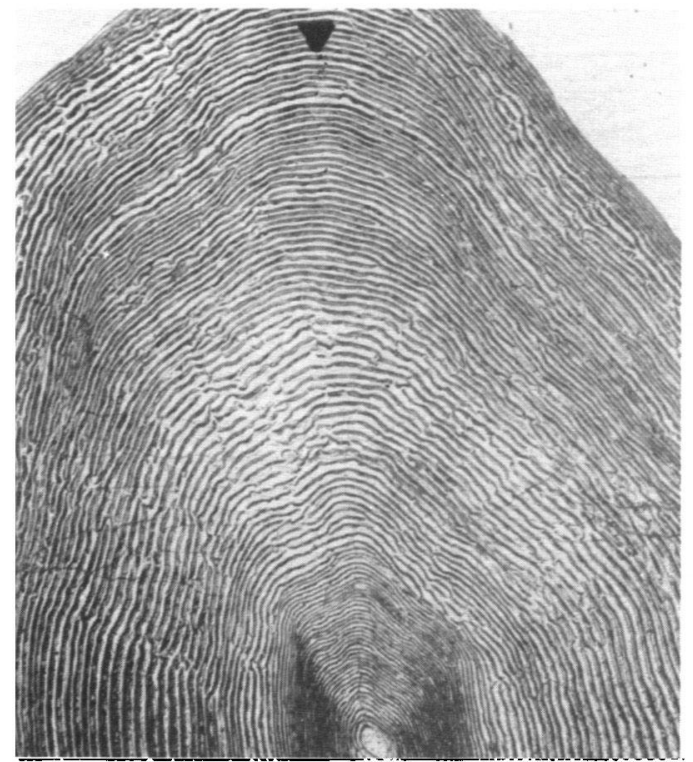

Figure 77 : Ellé, Bretagne, France, 29.03.75, ligne, 880, 1.2, poisson marqué comme smolt en 73, dédoublement du pre. mier hiver en mer ( $\$$ ).

Figure 77 : Ellé, Brittany, France, 29.03.75, rod, $880,1.2$, tagged fish as smolt in 73, Dividing of first sea winter into two parts ( $)$.

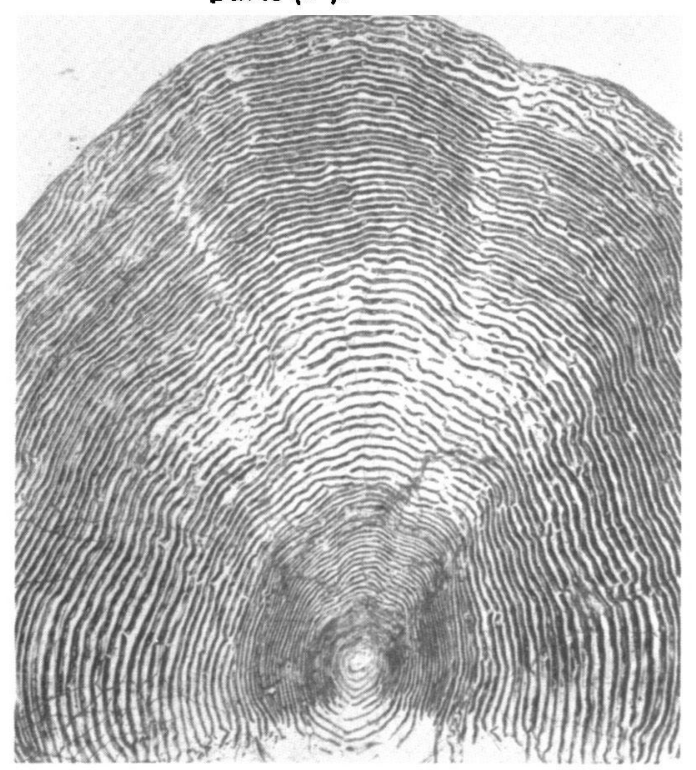

Figure 79 : Ellé, Bretagne, France, 26.03.75, ligne, 755, (d), 2.2. poisson marqué en 73 comme smolt, dédoublement important du premier hiver en mer.

Figure 79 : Ellé, Brittany, France, 26.03.75, rod, 755, (c), 2.2 , tagged as smolt in 73 , large dividing of first sea winter into two parts.

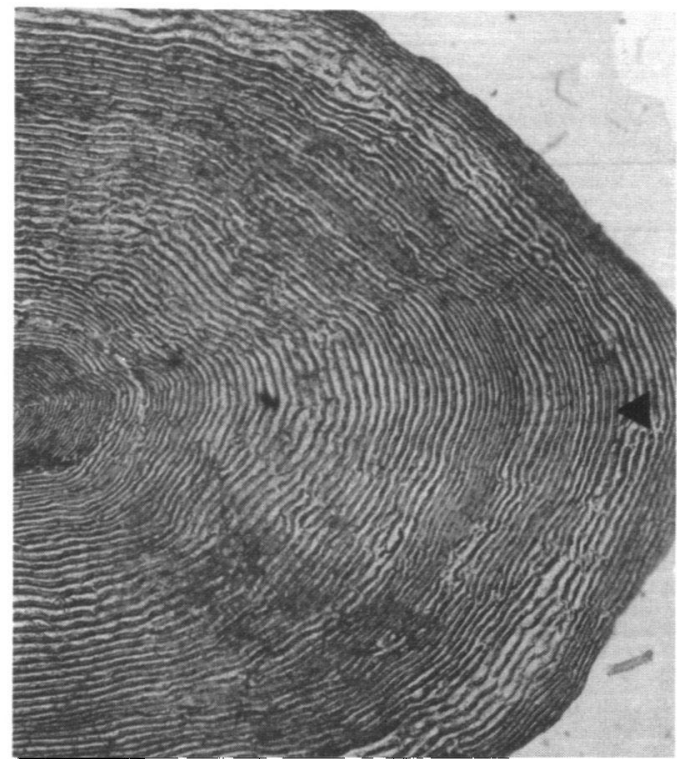

Figure 78 : Loire, Centre, France, 18.04.77, filet, 805, 1.2. dédoublement du premier hiver en mer ( ) .

Figure 78 : Loire, Centre, France, 18.04.77, net, 805, 1.2, dividing of first sea winter into two parts ( $(-)$

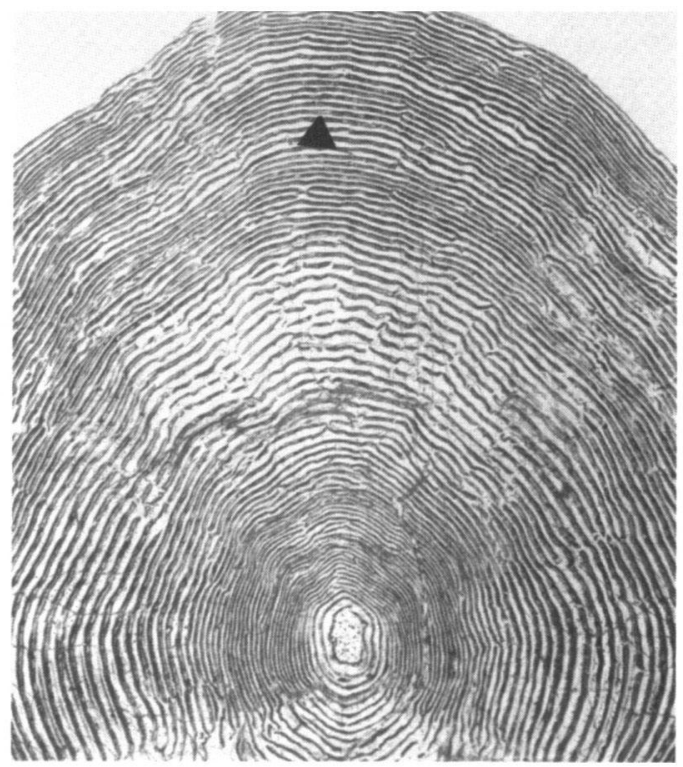

Figure 80 : Elorn, Bretagne, France, 28.05.84, ligne, 740, 2.2. poisson d'élevage marqué en 82. présence d'un check entre les deux hivers de mer ( $\downarrow$ ).

Figure 80 : Elorn, Brittany, France, 28.05.84, rod, $740,2.2^{+}$, rearing fish tagged in 82 , presence of check between the two sea winter $(\nabla)$. 


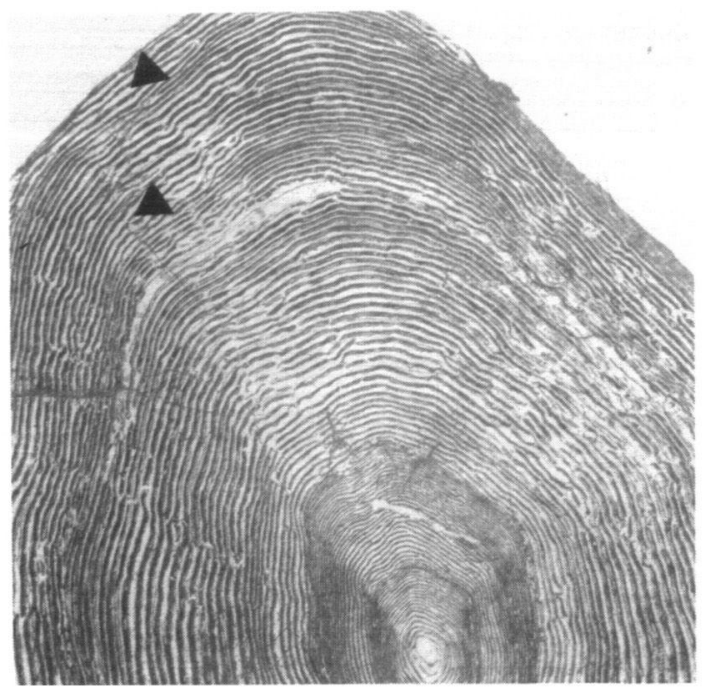

Figure 81 : Jet, Bretagne, France, 22.02.75, ligne, 730, 2.2, présence de deux checks entre le premier hiver en mer et le bord de l'écaille (\$).

Figure 81 : Jet, Brittany, France, 22.02.75, rod, 730, 2.2, presence of two checks between first sea winter and scale edge (D).

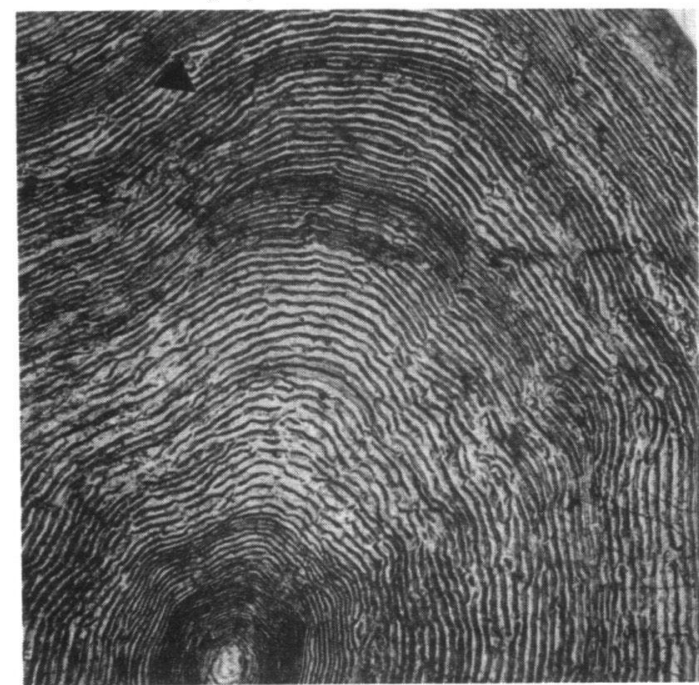

Figure 83 : Allier, Centre, France, 12.04.77, ligne, 950, 1.3, check entre le premier et deuxième hiver de mer ( $\downarrow$ ). compte tenu de sa position et du faible nombre de circuli, il ne peut pas être confondu avec un hiver.

Figure 83 : Allier, Centre, France, 12.04.77, rod, $950,1.3$, check between the first and second sea winter $(D)$. It cannot be a winter band according to its position and little circuli number.

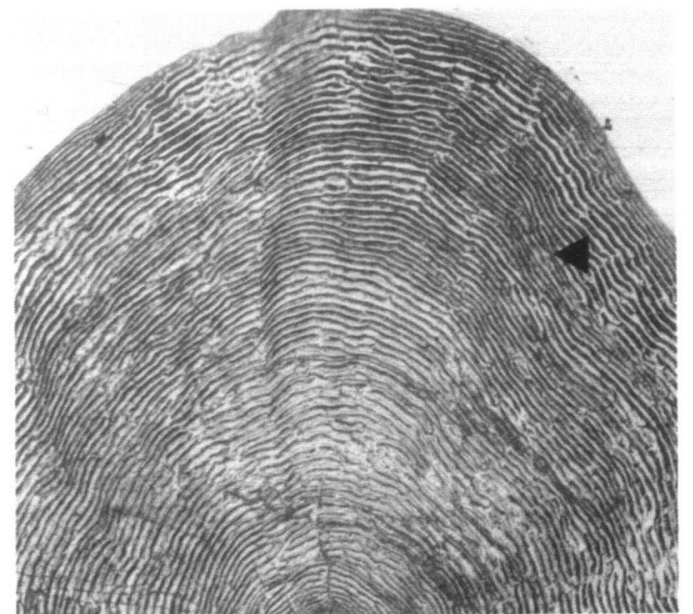

Figure 82 : Ellé. Bretagne, France, 23.03.75, ligne, 860, 1.2, poisson marqué en 73 comme smolt, check entre le premier et le deuxième hiver en mer $(-)$. peut prêter à confusion avec un trois ans de mer.

Figure 82 : Ellé, Brittany, France, 23.03.75, rod, 860, 1.2, tagged fish as smolt in 73, check between the first and second sea winter ( $D$ ), could be considered like a three sea winter fish.

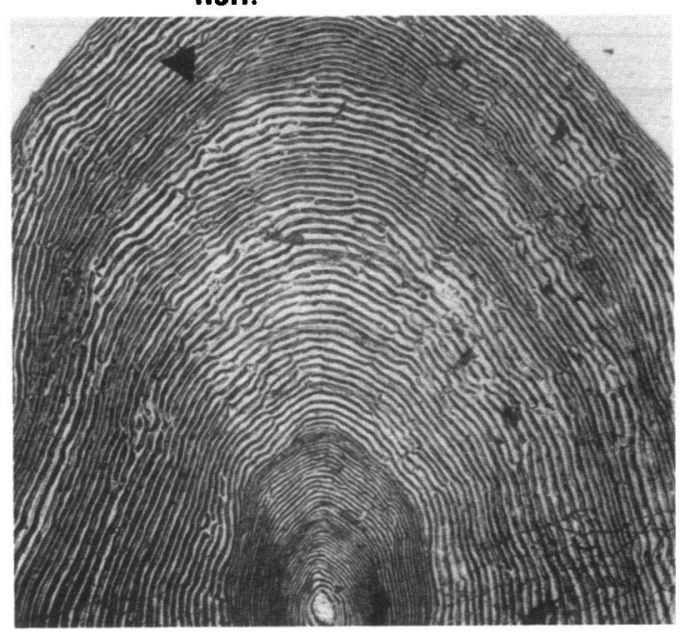

Figure 84 : Elorn, Bretagne, France,

3.04.77, ligne, 790, 2.2 probable, deuxième resserrement (-) mal positionné par rapport au premier et en tant que premier hiver donnerait une taille à un an de mer de $65.3 \mathrm{~cm}$.

Figure 84 : Elorn, Brittany, France, 3.04.77, rod, 790, 2.2 probably, the second narrow circuli band $(D)$ is badly located with regard to first sea winter. As a first winter band it would give a length of $65,3 \mathrm{~cm}$ at one sea year old. 


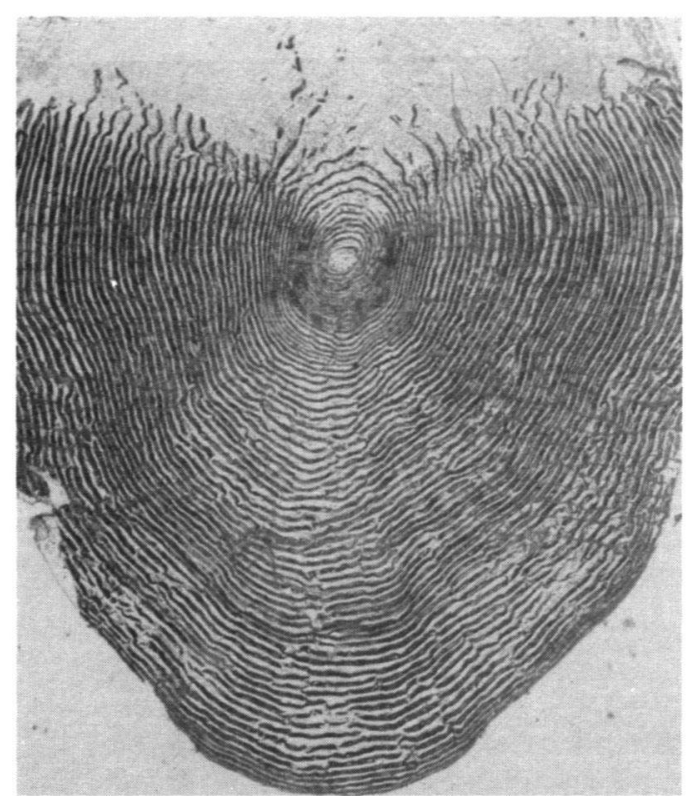

Figure 85 : Steir, Bretagne, France, 1.06.75, ligne, 640, 1.1 , présence de poux de mer, resserrement au bord de l'écaille.

Figure 85 : Steir, Brittany, France, 1.06.75, rod, 640, $1.1^{\dagger}$, presence of sea lice, narrow circuli band at the edge of scale.

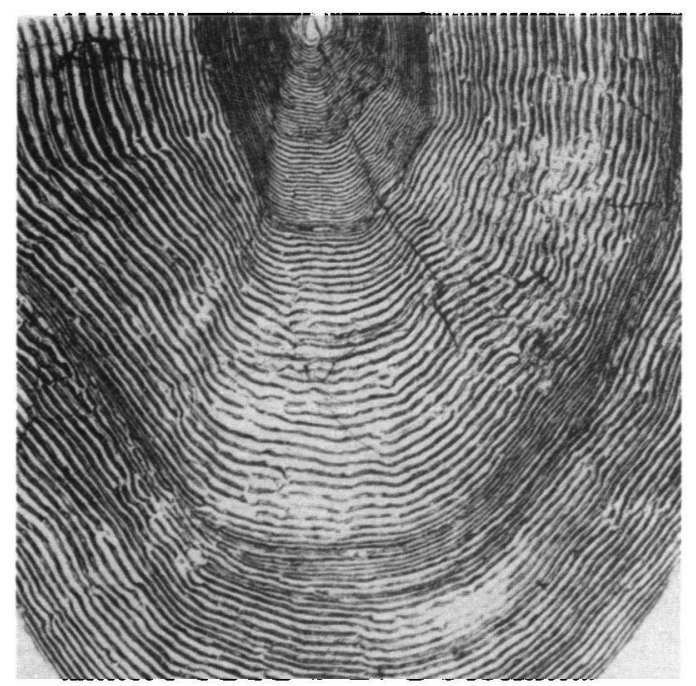

Figure 87 : Scorff, Bretagne. France, 7.03.81, ligne, 780, 2.2, deuxième hiver non formé. phénomène très courant chez les poissons de 2 ans de mer capturés en début de saison dans cette région.

Figure 87 : Scorff, Brittany, France, 7.03.81, rod, 780, 2.2, the second winter band is no visible, a very frequent phenomenon in two sea winter salmon caught at the beginning of fishing season in this area.

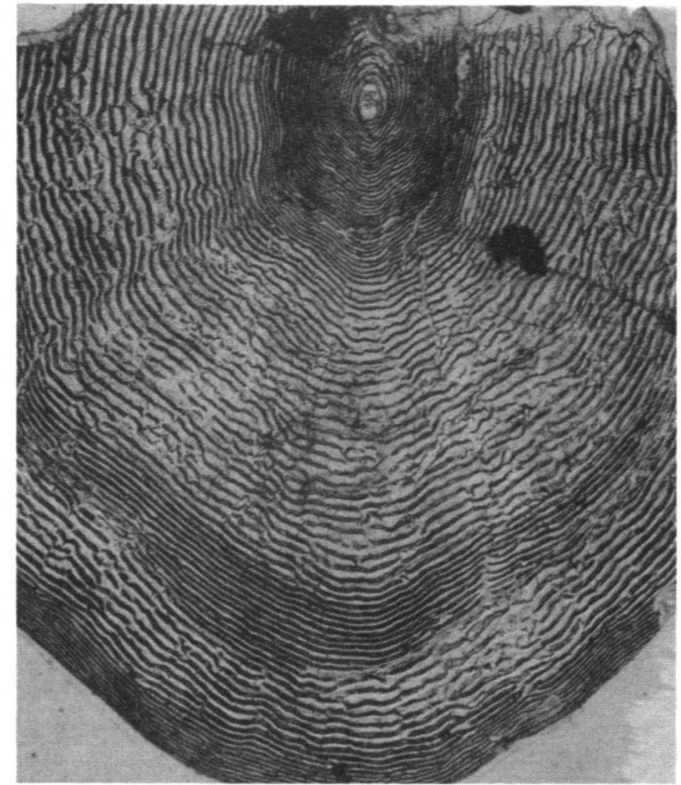

Figure 86 : Nivelle, Sud-Ouest, France, 23.09.84, piège de montée, $662, q, 1.1^{+}$, resserrement plus prononcé au bord de l'écaille.

Figure 86 : Nivelle, South West, France, 23.09.84, upstream trap, 662 , $9.1^{+}$distinct narrow circuli band at the scale edge.

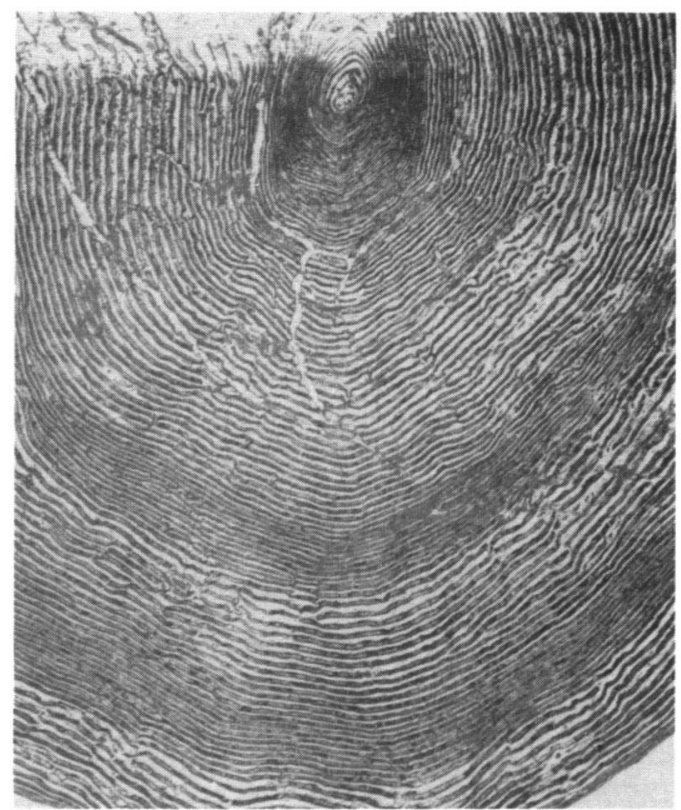

Figure 88 : Allier, Centre, France,

24.03.73, ligne, 960, 1.3, troisième hiver non formé. phénomène très courant chez ce type de poisson sur cette rivière.

Figure 88 : Allier, Centre, France,

24.03.73, rod, 960, 1.3, the third winter band is no visible, a very frequent phenomenon in this kind of fish on this river. 


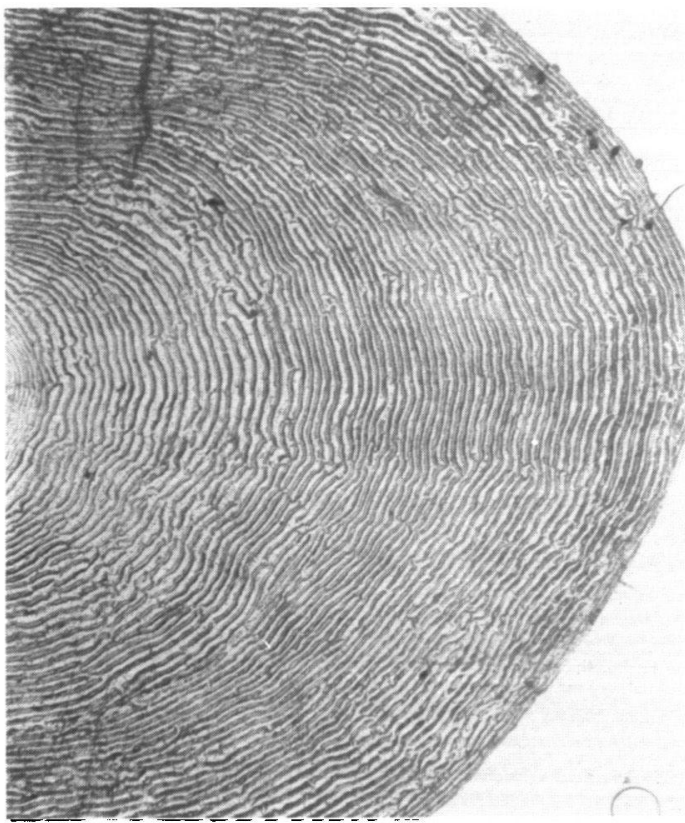

Figure 89 : Ellé, Bretagne, France, 22.02.75, ligne, 805, 1.2, poisson marqué comme smolt en 73, premier hiver en mer indiscernable.

Figure 89 : Ellé, Brittany, France, 22.02.75, rod, 805, 1.2. tagged fish as smolt in 73, first sea winter band no visible.

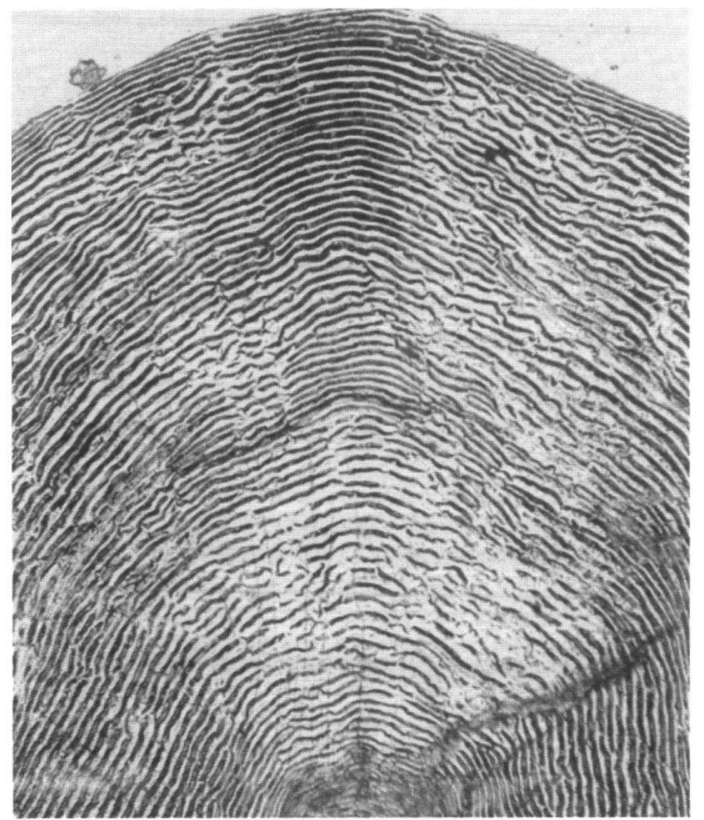

Figure 91 : Jet, Bretagne, France, 8.04.81, ligne, 880, 2.3 probable. deuxième hiver en mer difficilement discernable.

Figure 91 : Jet, Brittany, France, 8.04.81, rod, $880,2.3$, three sea winter fish probably, second sea winter band difficult to define.

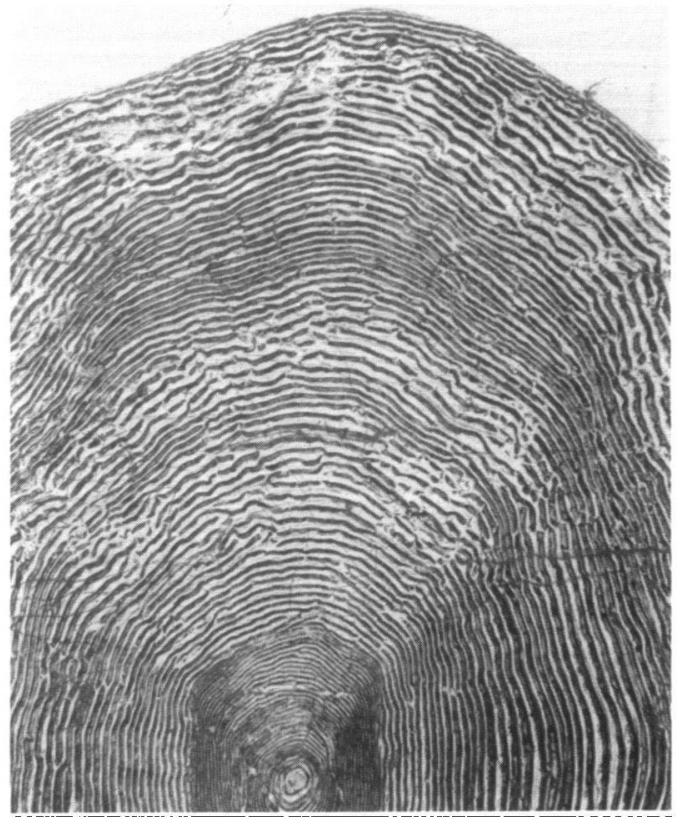

Figure 90 : Elorn, Bretagne, France, 24.03.77, ligne, 920, 2.3 probable à cause de sa taille, premier hiver difficile à définir.

Figure 90 : Elorn, Brittany, France, 24.03.77, rod, 920, 2.3, three sea winter fish probably according to length, first sea winter band difficult to locate.

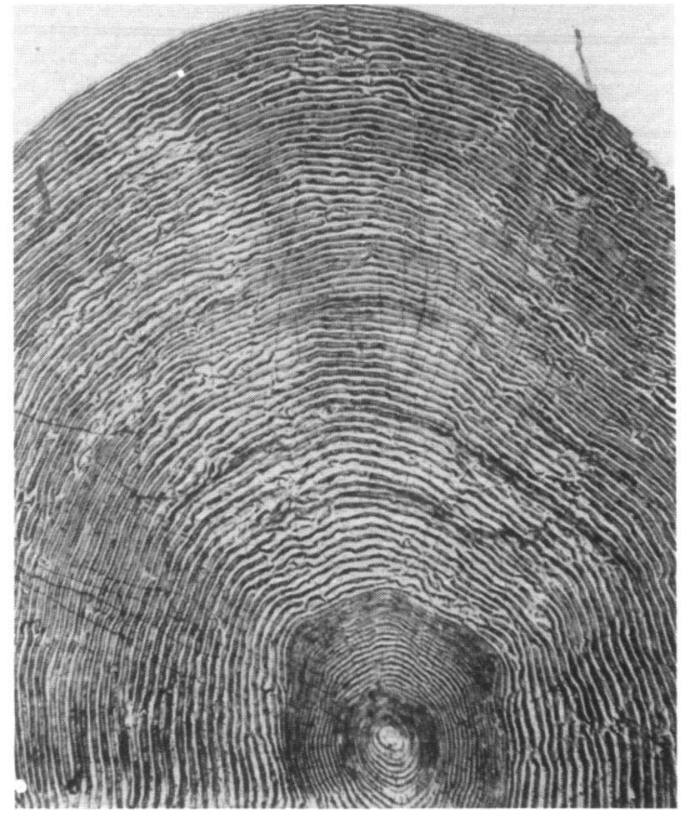

Figure 92 : Elorn, Bretagne, France, 15.03.80. ligne. 855, 2.2 ou 3 , aucun resserrement $n$ 'est correctement localisable.

Figure 92 : Elorn, Brittany, France, 15.03 .80 , rod, $855,2.2$ or 3 , no winter band can be correctly located. 


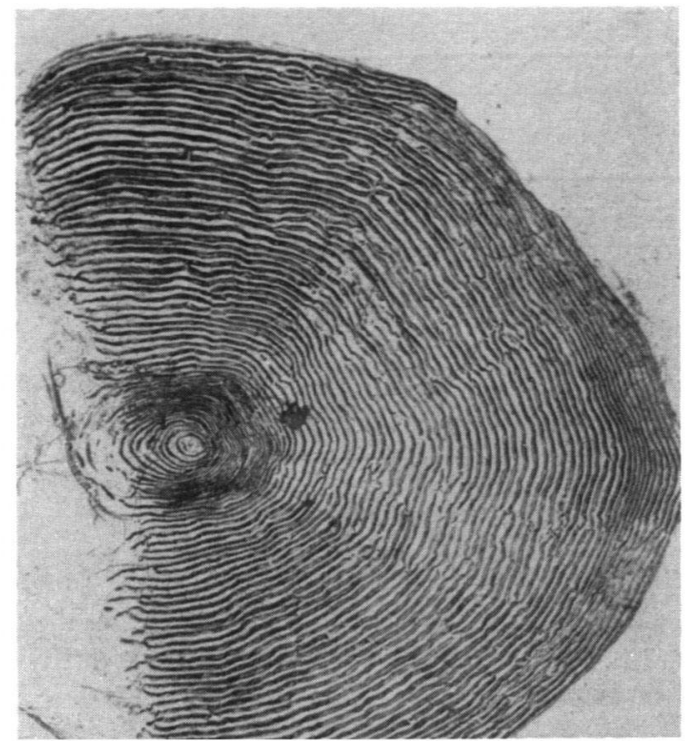

Figure 93 : Steir, Bretagne, France. 28.05.75, ligne, 580, $1.1^{+}$. castillon à très faible reprise de croissance après le premier hiver.

Figure 93 : Steir, Brittany, France, 28.05.75, rod, 580, 1.1 . grilse with very little plus growth after the first sea winter.

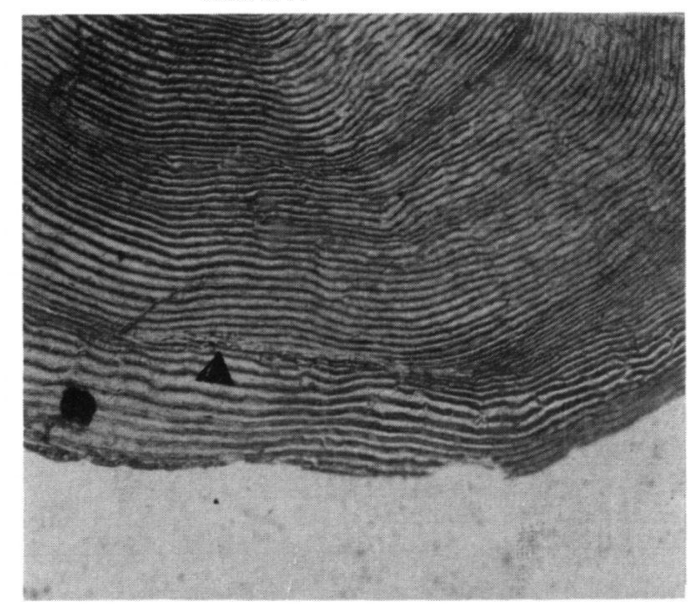

Figure 95 : Allier, Centre, France, 17.03.84, ligne, 1020, 1.3. érosion lors du deuxième hiver en mer ( $)$ ne peut être considérée comme une marque de fraie, phénomène également constaté chez le même type de poisson sur la rivière espagnole Esva.

Figure 95 : Allier, Centre, France, 17.03.84, ligne, $1020,1.3$. erosion at the second sea winter $(\triangleright)$, it is not a spawning mark, a phenomenon observed also in the same kind of fish on the spanish Esva River.

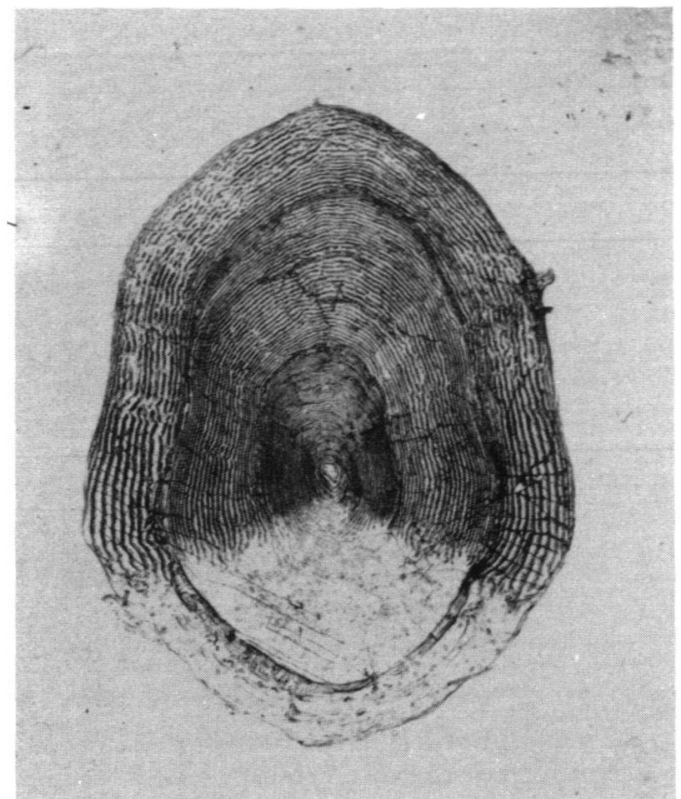

Figure 94 : Elorn. Bretagne. France, 8.04.77, ligne, 830, 2.2, présence d'une fausse marque de fraie faisant le tour de l'écaille.

Figure 94 : Elorn, Brittany, France, 8.04.77, rod, 830, 2.2, presence of false spawning mark around the scale.

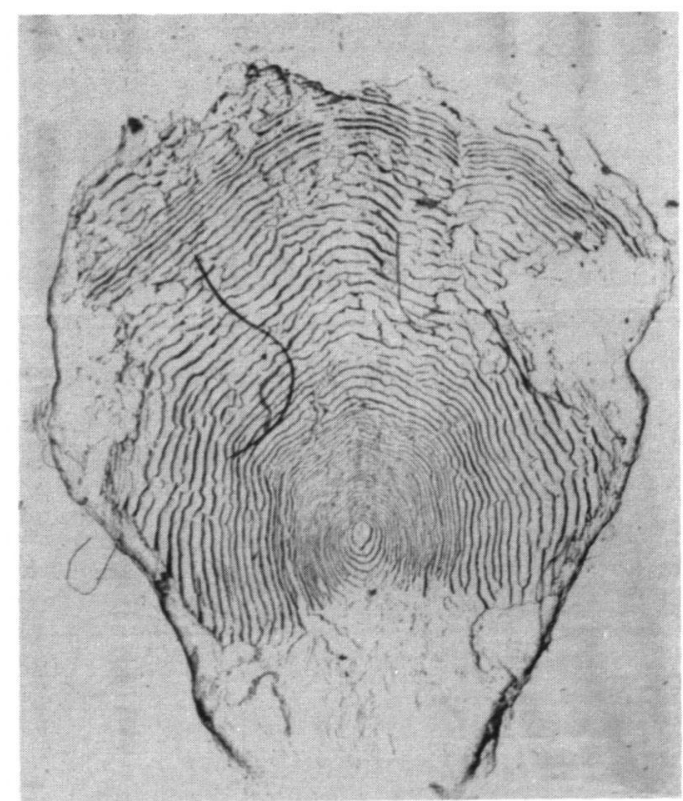

Figure 96 : Sélune (L'Oir). BasseNormandie, France, 18.11.84, piège de montée, 706, $\sigma^{\prime}, 1.1^{+}$ très forte érosion précédant la fraie, typique chez un mâle.

Figure 96 : Sélune (L'Oir), Low Normandy. France, 18.11.84, upstream trap, 706, $\sigma^{x}, 1.1^{+}$, very strong erosion of scale before spawning, typical in male. 


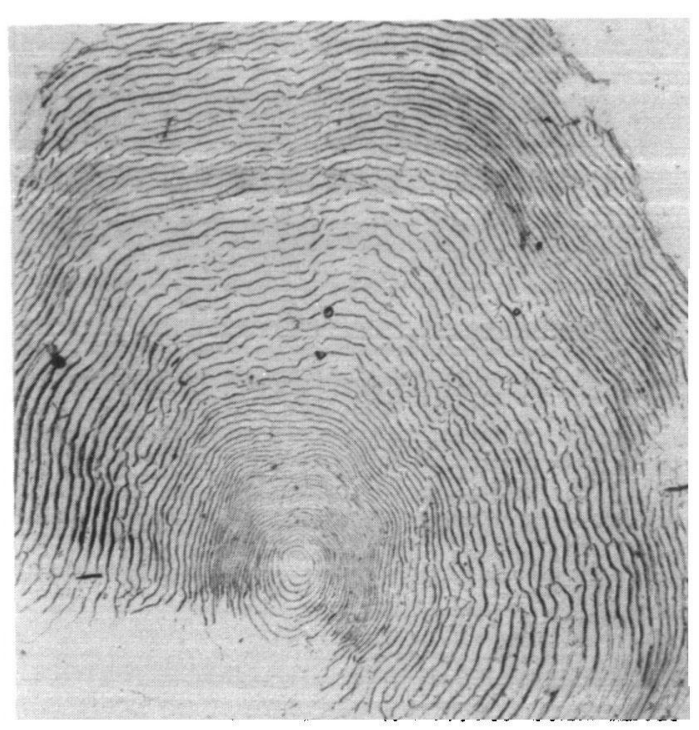

Figure 97 : Sélune (L'Oir), BasseNormandie, France, 18.11.84, piège de montée, 670, ㅇ. 1.1 ${ }^{\dagger}$, érosion précédant la fraie plus faible chez une femelle.

Figure 97 : Sélune (L'Oir), Low Normandy. France, 18.11.84, upstream trap, $670,9,1.1^{+}$, scale erosion before spawning less important in female.

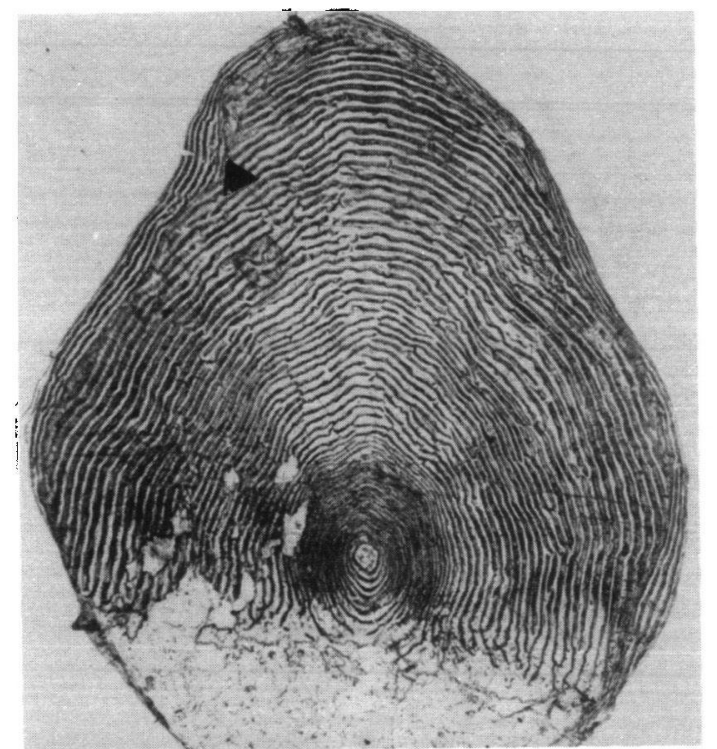

Figure 99 : Ellé, Bretagne, France, 2.04.73, piège de descente. $660.9,1.1^{+}$, becard de descente, reprise de croissance, marque de fraie visible ( () ), structure de l'écaille assez proche de celle de la Figure 98.

Figure 99 : Ellé, Brittany, France, 2.04.73. downstream trap, 660, mended kelt, plus growth after spawning mark ( $)$, scale structure fairly like that of Fig. 98.

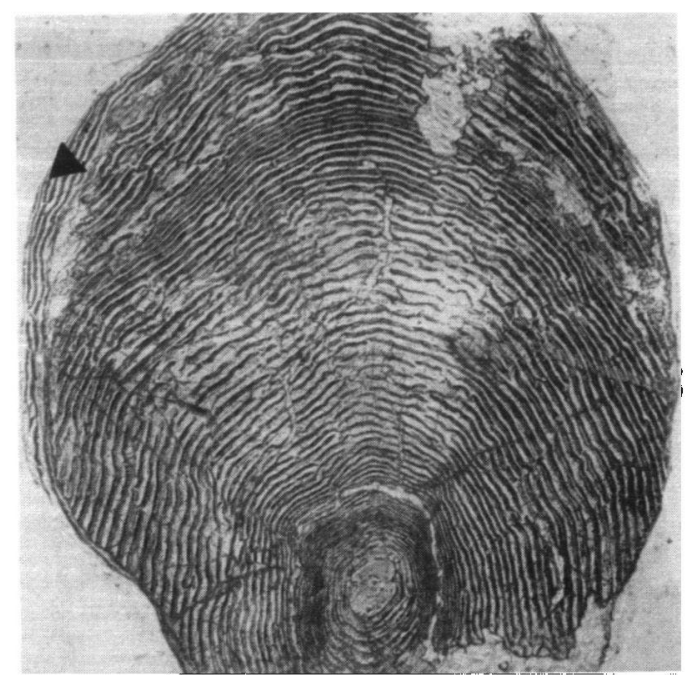

Figure 98 : Ellé, Bretagne, France, 3.10.82, piège de montée, $780,0^{7}, 1,2$ ème remontée. (marque de fraie $(\downarrow)$, première remontée comme castillon), présence de poux de mer, forte érosion à comparer avec Figure 99.

Figure 98 : Ellé, Brittany, France, 3.10.82, upstream trap, 780, $\sigma^{7}, 1$, previous spawner (spawning mark (-), first migration as grilse) presence of sea lice, strong erosion to compare with Figure 99.

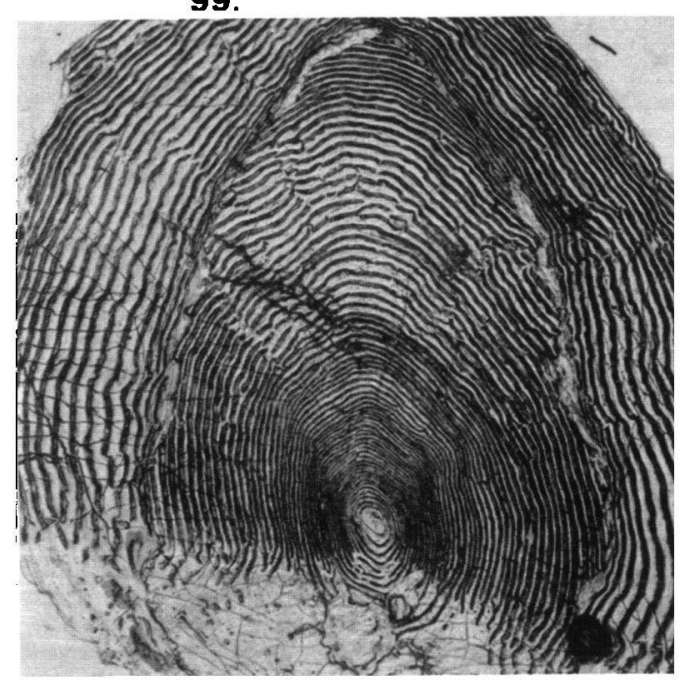

Figure 100 : Elorn, Bretagne, France,

1.03.80, ligne, 730, ‥ 1. poisson de deuxième remontée. présence d'une marque de fraie typique, âge de première remontée difficile à déterminer.

Figure 100 : Elorn, Brittany, France, 1.03.80, rod, 730 , ㅇ, 1. previous spawner, typical spawning mark, age at first migration difficult to know. 


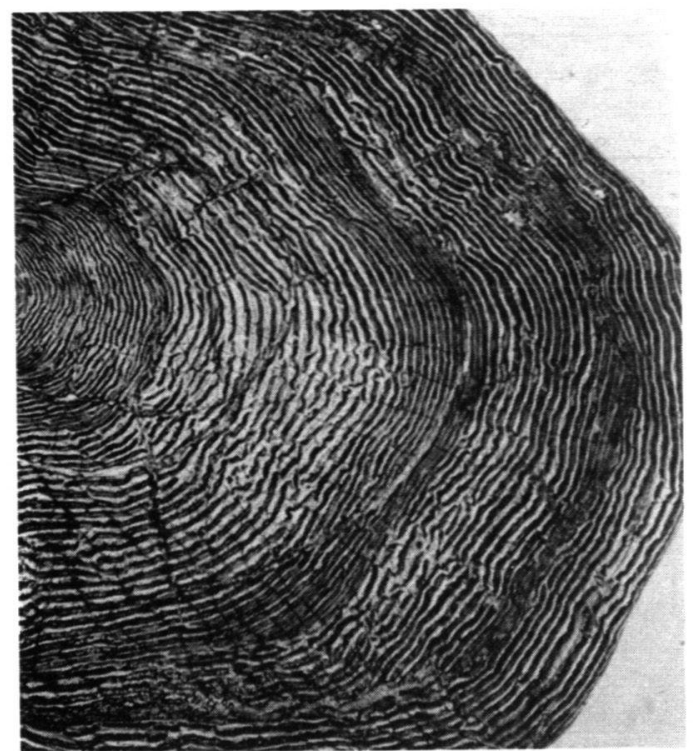

Figure 101 : Orne, Basse-Normandie. France, 5.08.81, piège de montee $695,+, 1$, poisson de deuxième remontée, âge probable de première remontée : $1^{+}$.

Figure 101 : Orne, Low Normandy, France, 5.08.81, upstream trap, 695 , , 1 , previous spawner, probably first migration as grilse.

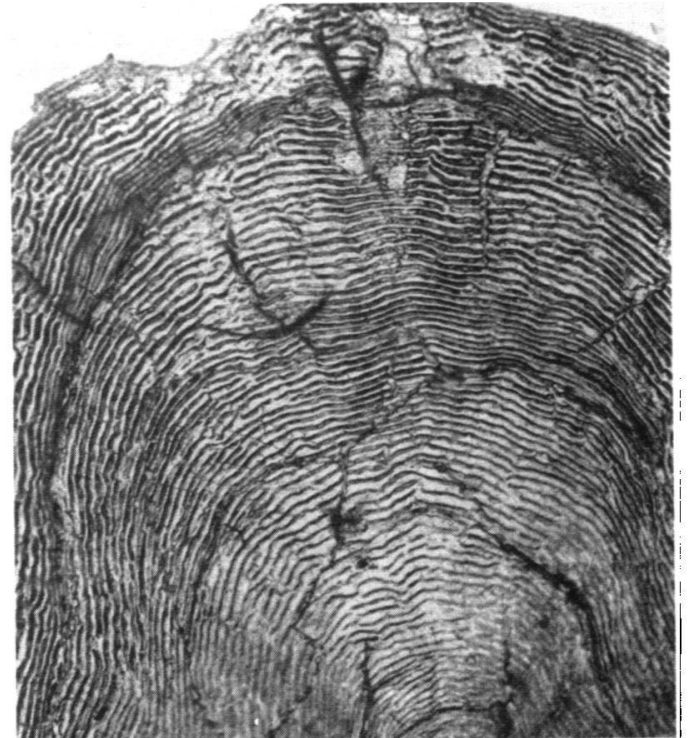

Figure 102 : Elle, Bretagne, France, 10.04.77, ligne, 900, $\delta^{\star}, 1$, poisson de 2 ème remontée marqué comme smolt en 73 . âge de première remontée : 2 ans.

Figure 102 : Ellé, Brittany, France, 10.04.77, rod, 900, $\sigma^{*}, 1$, previous spawner, tagged fish as smolt in 73, first migration as two sea winter fish.

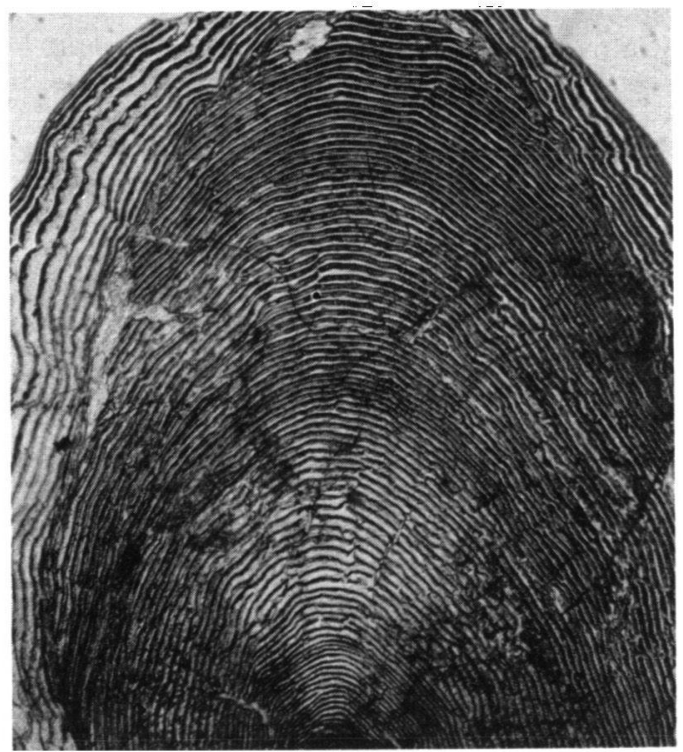

Figure 103 : Allier, Centre, France, 14.04.80, ligne, 1060 (ठا). 1 , poisson de deuxième remontée, âge probable de première remontée : 3 ans.

Figure 103 : Allier, Centre, France. 14.04.80, rod, $1060 .\left(\sigma^{7}\right) .1$. previous spawner, probably first migration as three sea winter fish. 


\section{4 - CONCLUSIONS ET RECOMMANDATIONS}

Un tel atelier a suscité un réel intérêt en raison de l'importance de la scalimétrie dans la connaissance des caractéristiques de base des populations de saumon atlantique. Ainsi, un certain nombre de recommandations est proposé à l'ensemble des organismes scientifiques et techniques (INRA, IFREMER, CSP, Consejeria de Agricultura y Pesca del Principado de Asturias) concernés par cette espèce dans son aire méridionale de répartition. Ces recommandations doivent s'appliquer à l'ensemble des rivières fréquentées par le saumon atlantique mais également à tous les bassins où des efforts de restauration sont actuellement entrepris (Garonne, Dordogne, Gartempe, Orne...). Ce sont : capture

1 - L'augmentation du nombre d'échantillons récoltés et des paramètres intéressant la

en période de pêche en préconisant la déclaration obligatoire des captures (réalisées en Espagne (MARTIN VENTURA, 1985) et sur l'Elorn, rivière du Finistère Nord, depuis 1981 (PROUZET, com. pers.), en voie de réussite dans les Côtes du Nord depuis 1984 (PORCHER, com. pers.)).

- en dehors de la période de pêche pour améliorer le niveau de connaissances sur les populations de fin d'été et d'automne.

Dans les deux cas un effort plus particulier doit être porté :

- sur la connaissance du rapport des sexes soit par prélèvement sanguin (LE BAIL et al.., 1981), soit par des critères externes (rapport de la longueur mâchoire/longueur totale, MAISSE \& BAGLINIÉRE, 1985). Ceci permettra d'estimer la potentiel reproducteur,

- sur les caractéristiques des bécards et des poissons de deuxième remontée.

- en rivière avec des inventaires annuels des populations de juvéniles sur des habitats caractérisés d'une manière homogène (BAGLINIËRE \& CHAMPIGNEULLE, 1982). L'état de maturation doit également être examiné.

2 - Une uniformisation de la récolte et du traitement des données avec :

- une localisation standard de prise d'écailles et leur montage sur film plastique par impression (presse de bijoutier et PVC de $1 \mathrm{~mm}$ d'épaisseur),

- la nécessité de constituer une collection annuelle centralisée de références d'écailles de juvéniles et d'adultes (écailies et/ou photos).

- I'utilisation d'un fichier informatique tel que celui mis au point par la délégation régionale Bretagne - Basse-Normandie du Conseil Supérieur de la pêche. Il permettra en retour d'informer les pêcheurs sur les caractéristiques de leur capture.

3 - La nécessité de poursuivre les recherches afin de comprendre les relations existant entre les structures scalimétriques et les facteurs de l'environnement aussi bien chez les populations sauvages que celles d'élevage (pisciculture, ruisseau, pépinière, étang ou lac). Pour ces dernières, cela nécessite entre autres le marquage au stade parr ou smolt pour qu'elles puissent bien être différenciées des populations sauvages. L'utilisation des écailles permet également d'aborder le problème de la discrimination des stocks par l'analyse discriminante en prenant en compte :

- soit leur forme par une technique d'analyses d'images (de PONTUAL, com. pers.).

- soit leurs structures internes en relation avec les caraçtéristiques biologiques (nécessité de connaître à tous les stades la relation longueur fourche - longueur totale).

La discrimination des stocks est une étape importante pour une gestion rationnelle des populations de saumon atlantique.

Ce dernier point constitue une des recommandations essentielles proposées par l'atelier international d'Aberdeen (BAGLINIERE, com. pers.).

D'un commun accord, l'atelier national se réunira annuellement pour débattre des problèmes posés par l'ensemble des connaissances acquises sur les populations de saumon atlantique (élevage et sauvage) dans son aire de répartition méridionale. II prendra le nom d'atelier "Scalimétrie et biologie du saumon atlantique". II devrait permettre :

- d'homogénéiser et de faire avancer les connaissances sur cette espèce,

- de constituer un point de départ pour la récolte de statistiques de pêche fiables (séparation saumon/castilion, captures mensuelles et par unité d'effort) qui seront publiées annuellement,

- de valoriser les positions française et espagnole dans la commission des poissons anadromes et catadromes et le groupe de travail du saumon de l'atlantique nord au sein du Conseil International pour l'Exploration de la Mer. 


\section{BIBLIOGRAPHIE}

BAGLINIERE J.L., 1976. Etude des populations de saumon atlantique (Salmo salar L., 1766) en Bretagne et Basse-Normandie. 1 - Caractéristiques des smolts de la rivière Ellé. Annales d'Hydrobiologie, $n^{\circ}$ 7. $141-158$.

BAGLINIERE J.L., PROUZET P., PORCHER J.P., NIHOUARN A. et MAISSE G., 1985. Caractéristiques générales des populations de saumon atlantique (Salmo salar L.) des rivières du Massif Armori-

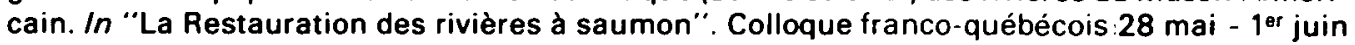
1985. Hydrobiologie et Aquaculture (sous presse).

BAGLINIÉRE J.L., CHAMPIGNEULE A., 1982. Densité des populations de truite commune (Salmo trutta L.) et de juvéniles de saumon atlantique (Salmo salar L.) sur le cours principal du Scorff (Bretagne) : préférendums physiques et variations annuelles (1976-1980). Acta Aecologica Oecol. Applic., 3 : 241-256.

BAGLINIÉRE J.L., MAISSE G., 1985. Precocious maturation and smoltification in wild atlantic salmon in the Armorican Massif, France. Aquaculture, 45, 249-263.

BILTON H.T., ROBINS G.L., 1971 a. Effects of feeding level on circulus formation on scales on young sockeye salmon (Oncorhynchus nerka). J. Fish. Res. Bd. Canada, 28 : $861-868$.

BILTON H.T., ROBINS G.L., $1971 \mathrm{~b}$. Effects of starvation, feeding and light period on circulus formation on scales of young sockeye salmon (Oncorhynchus nerka). J. Fish. Res. Bd. Canada, 28 : $1749-1755$.

BILTON H.T., ROBINS G.L., 1971c. Response of young sockeye salmon (Oncorhynchus nerka) to prolonged periods of starvation. J. Fish. Res. Bd. Canada, 28 : 1757-1761.

FOURNEL F., EUZENAT G., FAGARD J.L., 1985. Rivières à truite de mer et à saumons de Haute Normandie, réalités et perspectives. Le cas de la Bresle. In "La restauration des rivières à saumon". Colloque franco-québécois 28 mai-1 ${ }^{\text {er }}$ juin 1985. Hydrobiologie et Aquaculture (sous presse).

LEBAIL P.Y., BRETON B., MAISSE G., 1981. Détection de femelles de salmonidés en vitellogénèse. I Description de la méthode et mise en œuvre pratique. Bull. Fr. Piscic., 283 : 79-88.

MAISSE G., BAGLINIERE J.L., 1984. Mise en évidence d'une marque de fraie dans la phase eau douce des écailles de saumon atlantique du Massif Armoricain. ICES Comité des poissons anadromes et catadromes, $\mathrm{CM} 1983 / \mathrm{M}: 28,7 \mathrm{p}$.

MAISSE G., BAGLINIERE J.L., 1985. Le sexage morphologique du saumon atlantique (Salmo salar). Bull. Fr. Pêche Piscic. (sous presse).

MARTY A., BOUSQUET B., 1985. Le point sur les connaissances des populations naturelles de saumons du bassin de l'Adour. In "La restauration des rivières à saumon". Colloque franco-québécois 28 mai-1er juin 1985. Hydrobiologie et Aquaculture (sous presse).

MARTIN VENTURA J.A., 1985. Aportaciones al estudio del salmon atlantico ((Salmo salar L.) en los rios Esva, Narcea, Sella y cares del Principado de Asturias. Recursos Pesqueros de Asturias $n^{\circ} 5$ CRINAS. Consejeria de Agricultura y Pesca del Principado de Asturias (sous presse). 